\title{
Cosmic evolution of submillimeter galaxies and their contribution to stellar mass assembly ${ }^{\star}$
}

\author{
M. Michałowski ${ }^{1,2}$, J. Hjorth ${ }^{1}$, and D. Watson ${ }^{1}$ \\ 1 Dark Cosmology Centre, Niels Bohr Institute, University of Copenhagen, Juliane Maries Vej 30, 2100 Copenhagen Ø, Denmark \\ e-mail: michal@dark-cosmology.dk \\ 2 Scottish Universities Physics Alliance, Institute for Astronomy, University of Edinburgh, Royal Observatory, \\ Edinburgh, EH9 3HJ, UK
}

Received 10 November 2009 / Accepted 10 February 2010

\section{ABSTRACT}

\begin{abstract}
The nature of galaxies selected at submillimeter wavelengths (SMGs, $S_{850} \gtrsim 3 \mathrm{mJy}$ ), some of the bolometrically most luminous objects at high redshifts, is still elusive. In particular their star formation histories and source of emission are not accurately constrained. In this paper we introduce a new approach to analyse the SMG data. Namely, we present the first self-consistent UV-to-radio spectral energy distribution fits of 76 SMGs with spectroscopic redshifts using all photometric datapoints from ultraviolet to radio simultaneously. We find that they are highly star-forming (median star formation rate $713 M_{\odot} \mathrm{yr}^{-1}$ for SMGs at $z>0.5$ ), moderately dust-obscured (median $A_{V} \sim 2 \mathrm{mag}$ ), hosting significant stellar populations (median stellar mass $3.7 \times 10^{11} M_{\odot}$ ) of which only a minor part has been formed in the ongoing starburst episode. This implies that in the past, SMGs experienced either another starburst episode or merger with several galaxies. The properties of SMGs suggest that they are progenitors of present-day elliptical galaxies. We find that these bright SMGs contribute significantly to the cosmic star formation rate density $(\sim 20 \%)$ and stellar mass density $(\sim 30-50 \%)$ at redshifts 2-4. Using number counts at low fluxes we find that as much as $80 \%$ of the cosmic star formation at these redshifts took place in SMGs brighter than $0.1 \mathrm{mJy}$. We find evidence that a linear infrared-radio correlation holds for SMGs in an unchanged form up to redshift of 3.6, though its normalization is offset from the local relation by a factor of $\sim 2.1$ towards higher radio luminosities. We present a compilation of photometry data of SMGs and determinations of cosmic SFR and stellar mass densities.
\end{abstract}

Key words. galaxies: active - galaxies: evolution - galaxies: high-redshift - galaxies: ISM - galaxies: starburst submillimeter: galaxies

\section{Introduction}

Submillimeter galaxies (SMGs; see Blain et al. 2002) were discovered at $850 \mu \mathrm{m}\left(S_{850} \approx 3 \mathrm{mJy}\right)$ by the Submillimetre Common-User Bolometer Array (SCUBA; Holland et al. 1999) mounted on the James Clerk Maxwell Telescope (JCMT). Due to the coarse resolution of SCUBA, localizations derived from high-resolution radio maps had to be used to measure their spectroscopic redshifts (Chapman et al. 2005). Lots of studies have addressed the issue of characterizing the nature of SMGs (Egami et al. 2004; Greve et al. 2004, 2005; Smail et al. 2004; Swinbank et al. 2004, 2006, 2008; Takagi et al. 2004; Alexander et al. 2005; Borys et al. 2005; Kovács et al. 2006; Laurent et al. 2006; Pope et al. 2006; Tacconi et al. 2006, 2008; Takata et al. 2006; Younger et al. 2007, 2008, 2009a; Clements et al. 2008; Coppin et al. 2008; Dye et al. 2008, 2009; Hainline 2008; Hainline et al. 2009; Perera et al. 2008; Scott et al. 2008; Austermann et al. 2009; Devlin et al. 2009; Eales et al. 2009; Murphy et al. 2009; Murphy 2009; Tamura et al. 2009; Weiß et al. 2009b; Aravena et al. 2010, some of these works were based on surveys with sensitivity worse than $3 \mathrm{mJy}$ quoted above). However they were usually based on limited samples ( $\lesssim 20$ sources), limited wavelength coverage or photometric redshifts. These limitations have made it difficult to solve several issues, including the characterization of the star formation histories of SMGs and their dominant source of emission.

$\star$ Appendix is only available in electronic form at http: //www . aanda.org
An important open question concerns the contribution of SMGs to cosmic stellar mass assembly. This is important, because in order to understand galaxy evolution, the build-up of stellar mass must be mapped out to high redshifts. It is usually parametrized by the total star formation rate (SFR) density per unit comoving volume, $\left(\rho_{\mathrm{SFR}}\right.$; see e.g. Hopkins 2004 ; Hopkins \& Beacom 2006). At high redshifts it is difficult to disentangle the contribution to $\rho_{\mathrm{SFR}}$ from galaxy populations of different masses due to incompleteness at low luminosities.

Another approach to study stellar mass assembly is to consider directly the stellar mass density per unit comoving volume, $\rho_{*}$, which is equivalent to the integrated $\rho_{\text {SFR }}$ over the age of the Universe. It is established that $\rho_{*}$ grows with cosmic time (stellar mass is accumulating; Drory et al. 2005; Fontana et al. 2006; Elsner et al. 2008; Pérez-González et al. 2008; Marchesini et al. 2009), but the contribution from different galaxy populations is not well-determined. Spitzer observations of SMGs (Egami et al. 2004; Frayer et al. 2004; Ivison et al. 2004; Borys et al. 2005; Ashby et al. 2006; Laurent et al. 2006; Pope et al. 2006; Dye et al. 2008; Hainline 2008; Hainline et al. 2009) have enabled studies of the rest-frame near-infrared (near-IR) part of the spectrum, where old stellar populations are dominant - an important step forward in getting full spectral energy distributions and accurate estimates of stellar masses of SMGs. The results indicate that SMGs are among the most massive galaxies in the Universe.

The dominant source of emission from SMGs is dust reprocessed emission either from young stars or active galactic nuclei 
(AGNs). One way to test it is to compare the infrared (IR) and radio luminosities of SMGs, because, at least locally, star-forming galaxies follow a remarkably tight correlation between IR and radio luminosities (Helou et al. 1985; Condon 1992). The correlation is believed to result from the fact that both IR and radio emissions are related to short-lived massive stars: the former originates from dust heated by ultraviolet (UV) light from blue, massive stars and the latter from synchrotron emission of electrons produced in supernova remnants. Therefore, a relation consistent with the local one is an indication of star formation dominating both the IR and radio emissions. There is growing evidence that the correlation holds at redshifts $z \lesssim 1$ (Garrett 2002; Gruppioni et al. 2003; Appleton et al. 2004; Boyle et al. 2007; Marleau et al. 2007; Vlahakis et al. 2007; Yang et al. 2007). At higher redshifts sample sizes are small making it difficult to draw robust conclusions (Appleton et al. 2004; Kovács et al. 2006; Beswick et al. 2008; Ibar et al. 2008; Sajina et al. 2008; Garn et al. 2009; Murphy et al. 2009; Murphy 2009; Rieke et al. 2009; Seymour et al. 2009; Younger et al. 2009b; Sargent et al. 2010). The only sign of evolution was reported by Ivison et al. (2010) based on stacking analysis of the $24 \mu \mathrm{m}$-selected galaxies, though possibly interpreted as a selection effect.

The objective of this paper is to model for the first time the entire UV-to-radio spectral energy distributions of a statistically significant sample of SMGs in a self-consistent way. Using these models we i) consistently derive the properties of SMGs using all available data to characterize their nature and determine the dominant emission mechanism; ii) estimate the contribution of SMGs to the cosmic SFR and stellar mass densities; iii) investigate whether the local IR-radio correlation holds at high redshifts in an unchanged form. In Sect. 2 our SMG sample is presented. Our methodology is outlined in Sect. 3. We derive the properties of SMGs in Sect. 4 and discuss the implications in Sect. 5. Section 6 closes with our conclusions. We use a cosmological model with $H_{0}=70 \mathrm{~km} \mathrm{~s}^{-1} \mathrm{Mpc}^{-1}, \Omega_{\Lambda}=0.7$ and $\Omega_{m}=0.3$.

\section{Sample}

We base our analysis on 76 SMGs $\left(S_{850} \gtrsim 3 \mathrm{mJy}\right)$ from the sample of Chapman et al. (2005), all with spectroscopically measured redshifts spanning a range of 0.080-3.623.

The way the sample is selected involves complex biases, which are difficult to fully quantify and account for. The parent sample of Chapman et al. (2005) consists of 150 SMGs out of which 104 have radio identifications. The sample discussed here (76 galaxies) consists of the SMGs for which redshifts have been measured (spectroscopic completeness $\sim 75 \%$ ). All this implies that the sample is biased against: i) faint submillimeter emitters (low dust content and/or hot dust, influence mostly the low-z portion of the sample); ii) faint radio emitters (high- $z$ and cold dust, see Fig. 3 of Chapman et al. 2005); iii) faint optical emitters (difficult to obtain spectra); iv) $z \sim 1.2-1.8$ ("redshift desert" where no emission lines enter the observable wavelengths). At low redshifts $(z<1)$ the sample may also be incomplete due to a limited sky area (and therefore - volume) coverage making it difficult to detect rare strong submillimeter emitters (for details on the SMG selection effects see also Fig. 2 of Blain et al. 2004 and discussion in Sect. 4.4 of Michałowski et al. 2008).

It is important to estimate what the influence of these selection effects on our results is. In total we analyse $\sim 50 \%(76 / 150)$ of the parent sample. Additionally, 25 radio-detected SMGs without spectroscopic redshifts have similar long-wavelength properties compared to the redshift sample (see Fig. 1 of

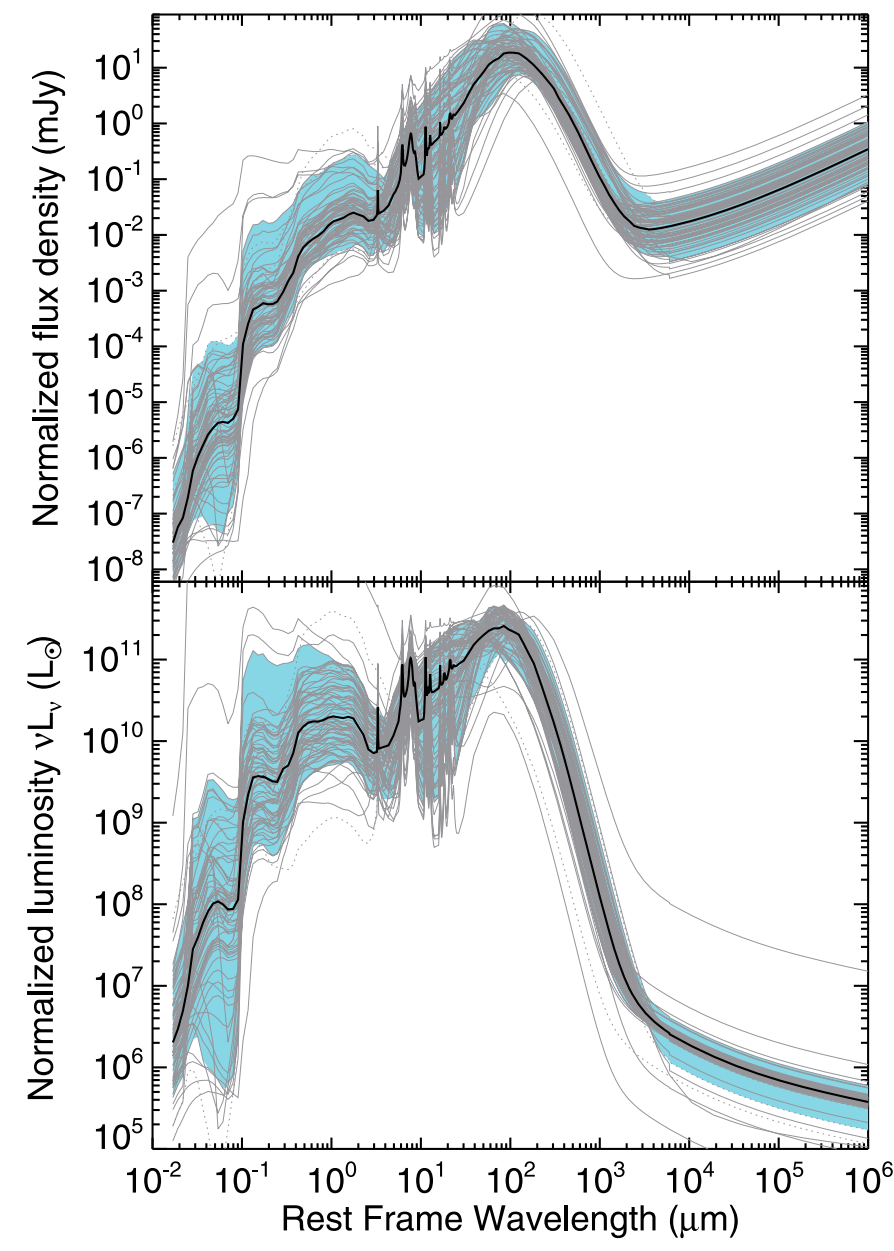

Fig. 1. Median spectral energy distribution (SED) of SMGs (thick lines) and SEDs of individual SMGs (thin lines). Dotted lines indicate $z<0.5$ objects. Shaded areas enclose $90 \%$ of the SEDs. Top: all SEDs were divided by the corresponding $850 \mu \mathrm{m}$ datapoint and scaled, so that the median SED has a flux of $5 \mathrm{mJy}$ at the rest-frame $283 \mu \mathrm{m}$ (observed $850 \mu \mathrm{m}$ at $z=2$ ). Bottom: SEDs were normalized to an infrared star formation rate of $100 M_{\odot} \mathrm{yr}^{-1}$.

Chapman et al. 2005), so their absence from the sample probably does not significantly bias our results. The same is true for the SMGs in the "redshift desert", since they are missed not due to their inherent properties. The remaining 46 radio-nondetected SMGs $(\sim 30 \%)$ could in principle have very different properties than our sample resulting in a potential limitation in our analysis.

Even if most of the SMGs without spectroscopic redshifts are similar to those in our sample, the incompleteness at $z<1.8$ implies that the estimates of SMG densities (Sects. 5.3.1, 5.3.2 and 5.2.3) in the three low-redshift bins (see Sect. 3.2) are strict lower limits.

Due to the negative $K$-correction at submillimeter wavelengths, SMGs at $z \gtrsim 0.5$ form a sample with homogenous IR luminosity (Blain \& Longair 1996; Blain 1997). However, SCUBA sources at $z \lesssim 0.5$ belong to a different population of objects and are intrinsically fainter. The limited volume coverage at these low redshifts makes the sample of these objects small and incomplete. This prevents a separate study of their properties. We did not take into account these sources when we computed median values of the properties of SMGs. 


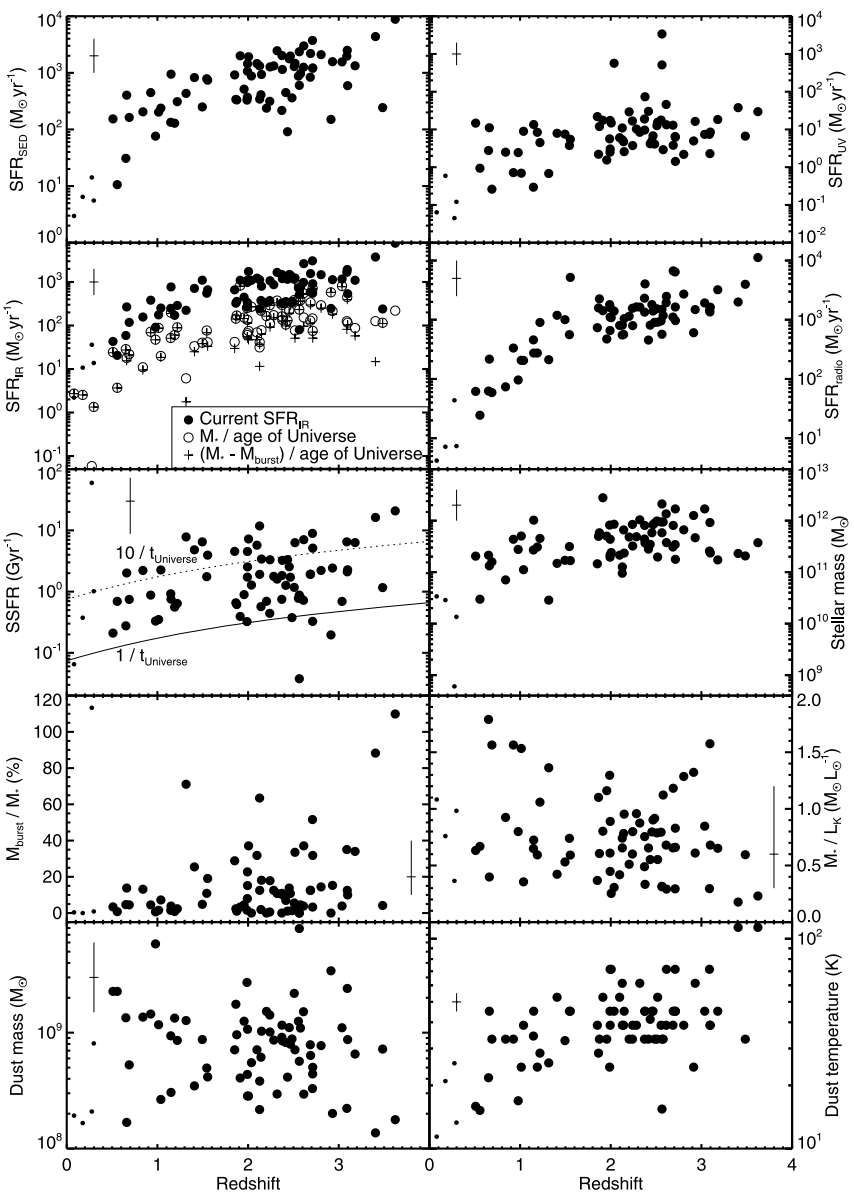

Fig. 2. Redshift evolution of the properties (full circles, see Table A.3 in Appendix) of the sample of 76 SMGs with spectroscopic redshifts (Chapman et al. 2005). Small symbols indicate $z<0.5$ objects. Typical errors (Sect. 4) are shown as crosses. From top-left to bottom-right: star formation rate (SFR) derived from spectral energy distribution modeling, ultraviolet, infrared and radio emission, SFR per unit stellar mass $\left(\equiv \mathrm{SFR}_{\mathrm{IR}} / M_{*}\right)$, stellar mass, fraction of stellar population formed during the ongoing starburst, stellar mass-to-light ratio, dust mass and temperature. In the SFR IR panel, we also show the minimum average SFRs (see Sect. 5.2.1) required to build up the total stellar mass within the age of the Universe at a given redshift (empty circles) and to build up the fraction of stellar population that was not formed during the ongoing starburst (plus signs). The location of plus signs indicates that SMGs must have been highly star-forming even before the onset of the ongoing starburst. When empty circles and plus signs overlap, the contribution of the ongoing starburst to the total stellar mass of a galaxy is negligible (i.e. $M_{\text {burst }} / M_{*} \sim 0$ ).

The photometric datapoints (Tables A.1 and A.2 in Appendix $\left.{ }^{1}\right)$ were collected from the literature: Ivison et al. (2002, $I K$, radio), Ivison et al. (2005, $R, 1.2 \mathrm{~mm})$, Chapman et al. (2003b, VI), Chapman et al. (2005, BR, $850 \mu \mathrm{m}$, radio), Capak et al. (2004, UBVRIzHK), Clements et al. (2004, $U B V I K)$, Egami et al. (2004, $24 \mu \mathrm{m})$, Greve et al. (2004, $1.2 \mathrm{~mm}$ ), Smail et al. (2004, IJK), Fomalont et al. (2006, $R z$ ), Kovács et al. (2006, $350 \mu \mathrm{m}, 1.2 \mathrm{~mm}$ ), Laurent et al. (2006, $350 \mu \mathrm{m}, 1.1 \mathrm{~mm})$, Tacconi et al. (2006, $1.3 \mathrm{~mm})$, Pope et al. (2006, R, $24 \mu \mathrm{m})$, Huynh et al. (2007, $160 \mu \mathrm{m})$, Hainline $(2008,3.6,4.8,5.6,8.0,24,70 \mu \mathrm{m})$. We have not used the existing mid-IR spectra (Valiante et al. 2007; Pope et al. 2008;

${ }^{1}$ For convenience we make the compilation available in electronic form. We suggest that the original data source be consulted and referred to appropriately.

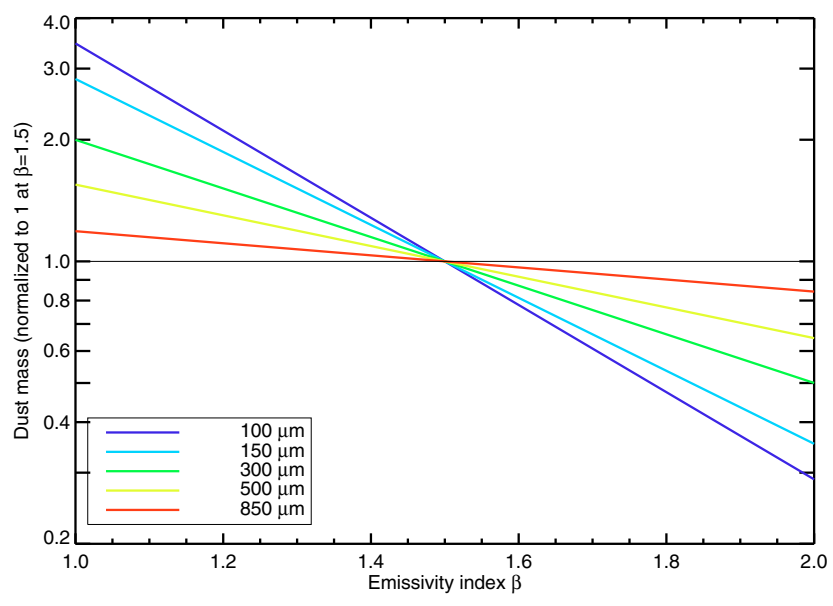

Fig. 3. Derived dust mass of a mock galaxy with dust temperature $T_{\mathrm{d}}=$ $40 \mathrm{~K}$ and a flux density of $5 \mathrm{mJy}$ at several infrared rest-wavelengths as a function of the assumed emissivity index $\beta$. For each wavelength the dust masses were normalized to 1 at $\beta=1.5$. The spread of the derived dust masses shows that the uncertainty of the dust mass resulting from unknown $\beta$ is a factor of a few.

Menéndez-Delmestre et al. 2007; Menéndez-Delmestre et al. 2009), but for completeness we have indicated in Table A.1 those SMGs for which Spitzer/IRS spectra exist.

\section{Methodology}

\subsection{SED modeling}

In order to model the spectral energy distributions (SEDs) of SMGs, we use all the photometric datapoints simultaneously. This has the advantage that all the galaxy properties are derived consistently regardless of the wavelength regime in which those properties shape the SEDs (for example, recent star formation governs the UV and far-IR parts of a spectrum of a galaxy, whereas accumulated stellar mass is responsible for nearIR emission). Moreover in the full SED modeling no single datapoint drives the fit alone.

We utilized the set of 35000 models from Iglesias-Páramo et al. (2007) developed in GRASIL (Silva et al. 1998) ${ }^{2}$ based on numerical calculations of radiative transfer within a galaxy. They cover a broad range of galaxy properties from quiescent to starburst. Their star formation histories are assumed to be a smooth Schmidt-type law (SFR proportional to the gas mass to some power, see Silva et al. 1998, for details) with a starburst (if any) on top of that starting $50 \mathrm{Myr}$ before the time of the evolution of a galaxy at which the SED is computed. Additionally we fitted templates based on nearby galaxies (Silva et al. 1998) and gamma-ray burst host galaxies (Michałowski et al. 2008). We simultaneously used all the photometric datapoints from UV to radio (Tables A.1 and A.2). In cases where the data given by different authors were contradictory, we disregarded the obvious outliers. We scaled the SEDs to match the data and chose the one with the lowest $\chi^{2}$.

Based on the best fits we derived the properties of the galaxies as explained in Michałowski et al. (2008, 2009). In particular, SFRs, stellar $\left(M_{*}\right)$ and starburst $\left(M_{\text {burst }}\right)$ masses were given as output from GRASIL, rest-frame UV and $K\left(L_{K}\right)$ monochromatic luminosities were interpolated from the bestfitting SEDs, whereas IR luminosities $\left(L_{\mathrm{IR}}\right)$ were integrated in

${ }^{2}$ http://adlibitum.oat.ts.astro.it/silva/default.html 
Table 1. Mean values for SMGs in redshift bins.

\begin{tabular}{|c|c|c|c|c|c|c|c|c|}
\hline $\begin{array}{c}z \\
(1)\end{array}$ & $\begin{array}{c}\text { Volume } \\
\left(10^{6} \mathrm{Mpc}^{3}\right) \\
(2)\end{array}$ & $\begin{array}{c}\log \rho_{\mathrm{IR}} \\
\left(L_{\odot} \mathrm{Mpc}^{-3}\right) \\
(3)\end{array}$ & $\begin{array}{c}\rho_{\mathrm{SFR}} \\
\left(M_{\odot} \mathrm{yr}^{-1} \mathrm{Mpc}^{-3}\right) \\
(4)\end{array}$ & $\begin{array}{l}\% \\
(5)\end{array}$ & $\begin{array}{c}\log \rho_{*} \\
\left(M_{\odot} \mathrm{Mpc}^{-3}\right) \\
(6)\end{array}$ & $\begin{array}{l}\% \\
(7)\end{array}$ & $\begin{array}{c}q \\
(8)\end{array}$ & $\begin{array}{c}\log \rho_{\text {dust }} \\
\left(M_{\odot} \mathrm{Mpc}^{-3}\right) \\
(9)\end{array}$ \\
\hline $0.080-0.500$ & 0.12 & $7.03_{-0.14}^{+0.11}$ & $0.0018 \pm 0.0005$ & $5_{-2}^{+3}$ & $6.35_{-0.16}^{+0.21}$ & $1_{-0.2}^{+0.4}$ & $2.54 \pm 0.12$ & $4.06_{-0.06}^{+0.05}$ \\
\hline $0.510-1.316$ & 1.03 & $7.81_{-0.07}^{+0.06}$ & $0.0111 \pm 0.0016$ & $9_{-2}^{+2}$ & $7.16_{-0.08}^{+0.10}$ & $\begin{array}{l}-0.2 \\
5_{-1}^{+2}\end{array}$ & $2.52 \pm 0.06$ & $4.32_{-0.05}^{+0.00}$ \\
\hline $1.408-2.142$ & 1.63 & $8.12_{-0.05}^{+0.05}$ & $0.0228 \pm 0.0027$ & $11_{-3}^{+3}$ & $7.18_{-0.11}^{+0.08}$ & $11_{-3}^{+6}$ & $2.29 \pm 0.08$ & $3.84_{-0.04}^{+0.04}$ \\
\hline $2.148-2.565$ & 0.89 & $8.45_{-0.05}^{+0.05}$ & $0.0486 \pm 0.0054$ & $18_{-5}^{+3}$ & $7.61_{-0.08}^{+0.11}$ & $51_{-11}^{+3}$ & $2.27 \pm 0.09$ & $4.35_{-0.04}^{-0.04}$ \\
\hline $2.578-3.623$ & 2.17 & $8.30_{-0.05}^{+0.05}$ & $0.0341 \pm 0.0040$ & $20_{-4}^{+5}$ & $7.28_{-0.07}^{-0.08}$ & $31_{-7}^{+11}$ & $2.25 \pm 0.08$ & $3.86_{-0.03}^{-0.04}$ \\
\hline
\end{tabular}

Notes. Column (1): redshift range of the bins. Column (2): comoving volume of these bins (calculated in Sect. 3.2). Column (3): IR luminosty density of SMGs. Column (4): Resulting IR SFR density of SMGs (Sect. 5.3.1). Column (5): contribution of SMGs to the cosmic SFR density (calculated in Sect. 3.2). Column (6): stellar mass density of SMGs (Sect. 5.3.2). Column (7): contribution of SMGs to the cosmic $M_{*}$ density (calculated in Sect. 3.2). Column (8): mean (and error of the mean) IR-radio correlation parameter for SMGs (Sect. 5.4.1). Column (9): dust mass density of SMGs (Sect. 5.2.3). Columns (3-7) and (9) have been corrected for incompleteness by a factor of 3.5 (Sect. 3.2).

a range $8-1000 \mu \mathrm{m}$, UV and IR SFRs $\left(\right.$ SFR $_{\mathrm{IR}}$ was adopted for all subsequent calculations, because $\mathrm{SFR}_{\mathrm{UV}}$ is on average two orders of magnitude lower) were calculated using Kennicutt (1998), dust masses $\left(M_{\mathrm{d}}\right)$ were calculated from the $850 \mu \mathrm{m}$ detections using Eq. (5) of Michałowski et al. (2009) and radio SFRs were calculated from the $20 \mathrm{~cm}$ detections using the empirical formula of Bell (2003) (see Sect. 4.2 of Michałowski et al. 2009). Dust temperatures $\left(T_{\mathrm{d}}\right)$ were estimated by identifying the peak of the dust emission and assuming an emissivity index $\beta=1.3$. The average extinction in the rest-frame $V$-band was calculated from the unextinguished starlight given in GRASIL: $A_{V}=2.5 \log$ (unextinguished $V$-band starlight / observed $V$-band starlight). IR-radio correlation parameters were calculated according to the formula $q=$ $\log \left(L_{\mathrm{IR}}\left[L_{\odot}\right] / 3.75 \times 10^{12} / L_{v 1.4 \mathrm{GHz}}\left[L_{\odot} \mathrm{Hz}^{-1}\right]\right)$, where $L_{v 1.4 \mathrm{GHz}}$ is a rest-frame $1.4 \mathrm{GHz}$ luminosity density computed from the observed $1.4 \mathrm{GHz}$ flux assuming a spectral slope of -0.75 .

\subsection{Volume densities}

In order to calculate the SFR density, the stellar density and the dust mass densities per unit comoving volume, $\rho_{\mathrm{SFR}}, \rho_{*}$ and $\rho_{\text {dust }}$, we used the following angular areas for the submillimeter surveys (Table 1 of Chapman et al. 2005): CFRS-03: 60 arcmin $^{2}$ and CFRS-14: $48 \operatorname{arcmin}^{2}$ (Webb et al. 2003b), Lockman Hole: $122 \operatorname{arcmin}^{2}$ and ELAIS-N2: $102 \operatorname{arcmin}^{2}$ (Scott et al. 2002), HDF-N: $100 \operatorname{arcmin}^{2}$ (Chapman et al. 2001), SSA-13 and SSA22: $100 \operatorname{arcmin}^{2}$ each (Chapman et al. 2003a), totaling 632 $\operatorname{arcmin}^{2}$.

We divided our sample into four high-redshift bins (Table 1) with approximately the same number of SMGs plus an additional bin for $z<0.5$ sources (see Sect. 2). The densities in each bin were calculated as a sum of $\mathrm{SFR}_{\mathrm{IR}}$ (or $M_{*}$, or $M_{\mathrm{d}}$ ) of all SMGs in this bin divided by its comoving volume (a similar approach to calculate the SFR and number volume densities of SMGs was taken by Coppin et al. 2009; Daddi et al. 2009b; Younger et al. 2009a; Wang et al. 2009). The volumes (Col. 2) were found using the total area from the previous paragraph.

We removed the contribution of ten $\mathrm{SMGs}^{3}$, which were observed by SCUBA in the photometry mode (as opposed to the

\footnotetext{
3 SMMJ123553.26+621337.7, SMMJ123555.14+620901.7, SMMJ123600.10+620253.5, SMMJ123600.15+621047.2, SMMJ123606.85+621021.4, SMMJ123716.01+620323.3, SMMJ163706.51+405313.8, SMMJ221804.42+002154.4, SMMJ221806.77+001245.7
}

blank-field mapping mode) targeting optically-faint radio galaxies (Chapman et al. 2005). These objects fall outside the fields discussed here.

The method is therefore to analyse the fraction of the sky observed by SCUBA and estimate the number of SMGs and their volume densities. However, the true number of SMGs in our fields could be higher. On the other hand, regardless of the selection effects, the true number of SMGs in our fields cannot be lower than the number of SMGs in our sample. In turn, the true values of SFR and $M_{*}$ densities cannot be lower than the values we derive. Therefore our results on volume densities should be regarded as robust lower limits.

Having this in mind we note that the parent sample of Chapman et al. (2005) includes only $29 \%$ of all the SMGs detected in the used survey fields (compare with Scott et al. 2002; Webb et al. 2003b,a). Therefore even if we analysed the full parent sample the estimated densities would be conservative lower limits. We attempt to correct for this incompleteness by assuming that the parent sample of Chapman et al. (2005) is a fair representation of the total population. In this case our numbers should be multiplied by $3.5(\sim 1 / 29 \%)$. This correction should in principle be derived separately for each redshift bin, but the missing redshift information for the majority of the SMGs in the used survey fields makes such calculation impossible. We note that this correction does not remove the bias against SMGs that are faint at radio and optical wavelengths, as discussed in Sect. 2.

We have not applied a volume density correction for the AGN contribution, because it is at most minor. Even though a fraction of SMGs host AGNs and a few individual SMGs have been shown to exhibit a significant AGN contribution to their emission, it is established that on average AGN activity is responsible for at most $\sim 10-20 \%$ of the bolometric infrared emission of SMGs (Alexander et al. 2005, 2008; Menéndez-Delmestre et al. 2007; Menéndez-Delmestre et al. 2009; Valiante et al. 2007; Pope et al. 2008; Hainline et al. 2009; Murphy et al. 2009; Watabe et al. 2009). Therefore a potential error associated with the AGN contribution in our analysis of a statistically significant sample is smaller than the systematic uncertainty (e.g. $30 \%$ error of luminosity-SFR conversion; Kennicutt 1998).

The percentage contribution of SMGs to the SFR and $M_{*}$ densities (Cols. 5 and 7 of Table 1) was calculated as $\rho_{\mathrm{SMG}} /\left(\rho_{\mathrm{SMG}}+\rho_{\mathrm{other}}\right)$, where $\rho_{\mathrm{SMG}}$ is the density of SMGs at each redshift bin (Cols. 4 and 6 ) and $\rho_{\text {other }}$ is the density of other galaxies assumed to be an average of determinations (excluding lower limits) reported by other authors (Fig. 4; Tables A.4 and A.5 in appendix), for which the redshift ranges overlap with 
M. Michałowski et al.: Evolution of submillimeter galaxies

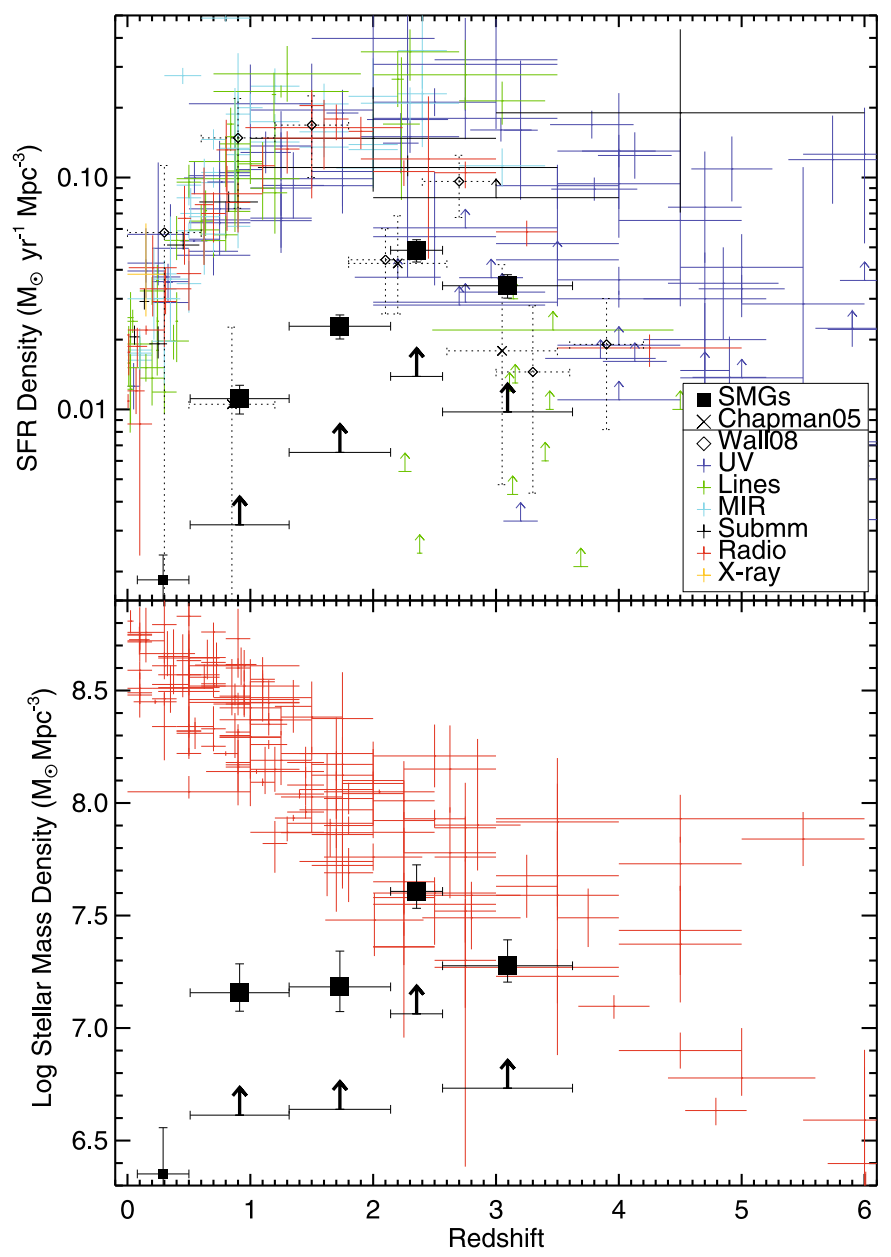

Fig. 4. Top: cosmic star formation density. The SMGs' contribution rises with redshift from $\sim 9 \%$ to $\sim 20 \%$ (Sect. 5.3.1 and Table 1). Filled Squares: data for SMGs at $z>0.5$ in four bins (Table 1 and Sect. 3.2). Small Squares: data for SMGs at $z<0.5$. Thick black arrows: the SMG data without incompleteness correction (factor of 3.5, Sect. 3.2). Black crosses and diamonds: star formation density of SMGs determined by Chapman et al. (2005) and Wall et al. (2008), respectively. Colored points with error bars: determination of the cosmic value based on different estimates - ultraviolet (violet), emission lines: [O II], [O III], $\mathrm{H} \alpha, \mathrm{H} \beta$ (green), mid-IR (light blue), submillimeter (black), radio (red), $\mathrm{X}$-ray (yellow). Extinction correction and, in many cases, incompleteness correction have been applied by the authors. Arrows: lower limits. Bottom: cosmic stellar mass density. The SMGs' contribution rises with redshift from $\sim 5 \%$ to $\sim 50 \%$ (Sect. 5.3.2 and Table 1). Red points with error bars: determination of the cosmic value from literature. The data and the references are listed in Tables A.4 and A.5 in Appendix.

our bins. This way of calculating the contribution is justified if SMGs do not enter the "other" samples of galaxies. This is usually the case because SMGs are faint in the optical. However, if this was not fulfilled, the real percentage contribution of SMGs would be even higher.

\section{Results}

The best fits ${ }^{4}$ are shown in Fig. A.1 and the median SEDs (in flux and luminosity domains) are shown in Fig. 1.

The resulting properties of the galaxies are listed in Table A.3 and shown in Fig. 2 as a function of redshift. We

${ }^{4}$ The SED fits can be downloaded from http://archive. dark-cosmology.dk notice similar trends to Hainline (2008) that lower- $z$ SMGs are less luminous and colder (see her Figs. 4.7 and 4.9).

In two cases we obtained much better fits using the templates of Silva et al. (1998) instead of those of Iglesias-Páramo et al. (2007), namely, an HR 10 template for SMMJ105151.69+572636.0 and a spiral Sc template for SMMJ221733.12+001120.2. In 9 cases $^{5}$ where our fits strongly underpredict the $850 \mu \mathrm{m}$ datapoint we adopted the $L_{\mathrm{IR}}$ and $T_{\mathrm{d}}$ estimates of Chapman et al. (2005).

The determination of the IR luminosity suffers from systematic uncertainties depending on the choice of the SED template. Our approach of using all the optical, submillimeter and radio data to constrain the shape of the SED results in a moderate systematic error in the IR luminosity (less than a factor of $\sim 2$; Bell et al. 2007). The choice of a Salpeter (1955) IMF with cutoffs of 0.15 and $120 M_{\odot}$ introduces a maximum systematic error of a factor of $\sim 2$ in the determination of the stellar masses and SFRs (Erb et al. 2006). Bell et al. (2007) have also found that random errors in stellar mass are less than a factor of $\sim 2$. Estimates of dust temperatures have uncertainties of $\sim 5-10 \mathrm{~K}$ dominated by the unknown value of the emissivity index, $\beta$. The SFR determination based on radio observations is accurate up to $30 \%$ since it agrees with the detailed spectrophotometric SED fitting (Michałowski \& Hjorth 2007). The uncertainties in $q$ (defined in Sect. 3.1) are $\sim 0.3$ (see also Kovács et al. 2006), dominated by the error in $L_{\mathrm{IR}}$.

In order to assess the influence of the choice of emissivity in$\operatorname{dex} \beta=1.3$ on the dust mass estimates, we recalculated the dust temperatures and masses in a range of $\beta$ of $1-2$. The resulting error was less than a factor of 3.5.

This is illustrated in Fig. 3 where we present a more systematic analysis of this problem. We calculated the dust mass of a mock galaxy with $T_{\mathrm{d}}=40 \mathrm{~K}$ (this choice does not influence the results) using $\beta$ in the range $1-2$ assuming a flux density of $5 \mathrm{mJy}$ at a variety of infrared rest-wavelengths probed by observations. Then we normalized dust masses to 1 at $\beta=1.5$. We conclude that as long as the observations probe wavelengths longer than $\sim 150 \mu \mathrm{m}(z \lesssim 4.7$ for observed wavelength of $850 \mu \mathrm{m})$, then the error on the dust mass resulting from unknown $\beta$ is less than a factor of $\sim 5$.

None of these errors significantly affects our conclusions, because the inferred nature of SMGs would not be different even in the worst case scenario when all systematic errors work in one direction (increasing or decreasing the obtained values). Moreover, we analyse a statistically significant sample of 76 galaxies, so random errors of a factor of 2 are reduced to $<20 \%$ when an error of a mean is considered.

Table 1 contains the volume densities and mean IR-radio correlation parameter divided into five redshift bins (see Sect. 3.2). The uncertainties quoted on $\rho_{\mathrm{SFR}}$ and $\rho_{*}$ include the systematic $30 \%$ uncertainty of the $L_{\mathrm{IR}}$ to SFR conversion (Kennicutt 1998) and a factor of $\sim 2$ systematic uncertainty in the stellar mass (Michałowski et al. 2008). The systematic error resulting from our incompleteness correction (Sect. 3.2) is likely a factor of a few.

\footnotetext{
5 SMMJ030226.17+000624.5, SMMJ030231.81+001031.3, SMMJ030236.15+000817.1, SMMJ030238.62+001106.3, SMMJ123636.75+621156.1, SMMJ123651.76+621221.3, SMMJ123721.87+621035.3, SMMJ163639.01+405635.9, SMMJ221724.69+001242.1
} 


\section{Discussion}

\subsection{Spectral energy distributions of SMGs}

We have presented the first successful attempt to fit the entire UV-to-radio SEDs of SMGs in a self-consistent way taking into account all the available data simultaneously. Our study provides evidence that GRASIL models can reproduce the SMG data. Namely, we found good fits for all SMGs in our sample with the best IR/submillimeter wavelength coverage ${ }^{6}$ except of SMMJ105238.30+572435.8.

As is evident from Fig. 1, regardless of whether SEDs were normalized to the same observed $850 \mu \mathrm{m}$ datapoint or $\mathrm{SFR}_{\mathrm{IR}}$, the scatter at optical and near-IR wavelengths is significant, showing that SMGs exhibit a wide range of stellar population properties (as also noted by Ivison et al. 2002). This implies the need for an SED template library in SMG studies, as opposed to singletemplate fitting.

Having constrained the SEDs of SMGs we now turn to a discussion of what we can learn about these galaxies using the best-fitting models.

\subsection{Properties of SMGs}

\subsubsection{Star formation rates}

The very high (current) SFRs of SMGs (median $713 M_{\odot} \mathrm{yr}^{-1}$, Col. 5 of Table A.3 and Fig. 2) place them among the most powerful starburst galaxies in the Universe. Such extreme SFRs likely result from major mergers (e.g. Chapman et al. 2004; Swinbank et al. 2004; Greve et al. 2005; Tacconi et al. 2006, 2008; Younger et al. 2007, 2008; Berciano Alba et al. 2010; Narayanan et al. 2009, 2010) and cannot be sustained for a long period (after a few hundred Myr at most the gas reservoir should be depleted; see Greve et al. 2005; Hainline et al. 2006).

On the other hand, their extinction-uncorrected UV SFRs are two orders of magnitude lower (median $\sim 7 M_{\odot} \mathrm{yr}^{-1}$, Col. 4). This implies that the majority of star formation in SMGs is hidden by dust. Therefore, optical observations alone are not sufficient to investigate their nature and contribution to cosmic star formation.

Using stellar masses of SMGs we placed lower limits on the time-averaged SFRs required to build their stellar masses within the age of the Universe ( $\equiv M_{*}$ /age of the Universe at given redshift), shown as empty circles in Fig. 2. Their median value of $\sim 130 M_{\odot} \mathrm{yr}^{-1}$ indicates that SMGs had to be relatively highly star-forming throughout the age of the Universe to build up their stellar populations at a constant rate. Even if our estimates of stellar masses were underestimated by a factor of a few due to systematic uncertainties (Sect. 4), the SMGs would have had to be luminous infrared galaxies (LIRGs with $\mathrm{SFR} \gtrsim 20 M_{\odot} \mathrm{yr}^{-1}$ ) during their evolution.

Having constrained the mass of stars formed during the ongoing starburst episode, $M_{\text {burst }}$, we can further constrain the minimum average SFR of SMGs before the onset of this starburst, $\equiv\left(M_{*}-M_{\text {burst }}\right) /$ age of the Universe (plus signs in Fig. 2). The median is still high, $\sim 100 M_{\odot} \mathrm{yr}^{-1}$, so SMGs must have been highly star-forming in the past too. At redshifts 2-3 the age of the Universe is $\sim 3-2$ Gyr and it is unlikely that a galaxy can sustain this high SFR over such a long period. Therefore we conclude that either the stellar masses of SMGs have been formed in

\footnotetext{
${ }^{6}$ SMMJ105201.25+572445.7, SMMJ105230.73+572209.5, SMMJ163650.43+405734.5, SMMJ163658.19+410523.8, SMMJ163706.51+405313.8
}

at least two strong $\left(>100 M_{\odot} \mathrm{yr}^{-1}\right)$ starburst episodes or continuously over the period of 2-3 Gyr but in several smaller galaxies that eventually merged. In order to build up the stellar mass of one SMG, five such galaxies would need to form stars continuously at a rate of $20 M_{\odot} \mathrm{yr}^{-1}$, a value more likely to be sustainable over several Gyr. The latter scenario is consistent with the results of Dye et al. (2008) based on observed optical to mid-IR data of 51 SMGs with photometric redshifts. They found that approximately half the stellar mass in SMGs has been formed over a long ( 1-2 Gyr) period of approximately constant star formation activity. The possibility that a significant part of stellar mass in SMGs was formed before the ongoing starburst has also been suggested by Hainline (2008), who compared the build-up timescale of stellar mass and the duration of the SMG phase.

The median value of the SFR per unit stellar mass (SSFR $\equiv \mathrm{SFR}_{\mathrm{IR}} / M_{*}$, Col. 7 of Table A.3) of $\sim 1.8 \mathrm{Gyr}^{-1}$ is within the range for other high- $z$ star-forming samples (compare with Figs. 2 and 4 of Castro Cerón et al. 2006, 2009, respectively). This indicates that SMGs are forming stars intensely.

SSFRs are compared with (the inverse of) the age of the Universe in Fig. 2. The SMGs close to the solid line could have formed their stellar populations at the present rate within the age of the Universe. However, the SMGs close to, or above the dashed line could have formed their stars at the present rate within less than $10 \%$ of the age of the Universe, i.e., within $\lesssim 300 \mathrm{Myr}$ at $z=2$. These galaxies are experiencing a powerful starburst episode.

At the extreme there are three high- $z \mathrm{SMGs}^{7}$ with very high SSFRs $>10 \mathrm{Gyr}^{-1}$ (Col. 7 of Table A.3). They are all hot ( $T_{\mathrm{d}}>60 \mathrm{~K}$, Col. 13) and formed the majority of their stellar populations during the ongoing starburst $\left(M_{\text {burst }} / M_{*}>60 \%\right.$, Col. 9). Therefore they are likely the most powerful cases of SMGs formed in major mergers of galaxies with huge gas reservoirs that were subsequently converted into stars.

Our median SSFR at $z>1.7\left(1.83 \mathrm{Gyr}^{-1}\right)$ is a factor of $\sim 2$ lower than that of Dunne et al. (2009, 3-4.5 $\mathrm{Gyr}^{-1}$; see their Fig. 12b) for $10^{11}<M_{*}<10^{12} M_{\odot}$ galaxies at these redshifts. This difference can be explained if the radio luminosities (used by Dunne et al. 2009, to estimate SFRs) are boosted by AGN activity more than the IR luminosities used here. Indeed, if we

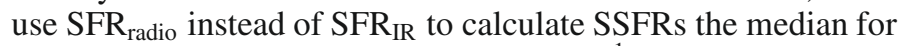
the SMGs at $z>1.7$ increases to 3.20 $\mathrm{Gyr}^{-1}$ (see Sect. 5.4.2 for discussion of AGN contamination in our sample).

In order to assess the accuracy of SFR estimates based on radio emission (independent of SED modeling) we compared the ratio of $\mathrm{SFR}_{\text {radio }} / \mathrm{SFR}_{\mathrm{IR}}$. Its median value is equal to $\sim 1.3$. Hence, assuming that IR emission is a good proxy for SFR, then radio estimates suffer from a $\sim 30 \%$ systematic error. This is illustrated in Fig. 5 where the dashed line denotes the relation between IR and radio luminosities required to make $\mathrm{SFR}_{\mathrm{IR}}=$ $\mathrm{SFR}_{\text {radio }}$. Indeed the radio luminosity gives systematically higher SFRs for SMGs (most of the points are above the line). This can be caused by a significant AGN contamination boosting radio flux (see Sect. 5.4.2), or a strong bias favouring radio-bright galaxies, because those non-detected at radio do not enter our sample (Sect. 2). Alternatively, it could be that for luminous galaxies either the IR conversion of Kennicutt (1998) should be scaled up by a factor of 1.3 , or the radio conversion of Bell (2003) scaled down.

\footnotetext{
7 SMMJ131201.17+424208.1, SMMJ141802.87+523011.1, SMMJ221806.77+001245.7 plus a low-mass, low- $z$ case, SMMJ030238.62+001106.3
} 


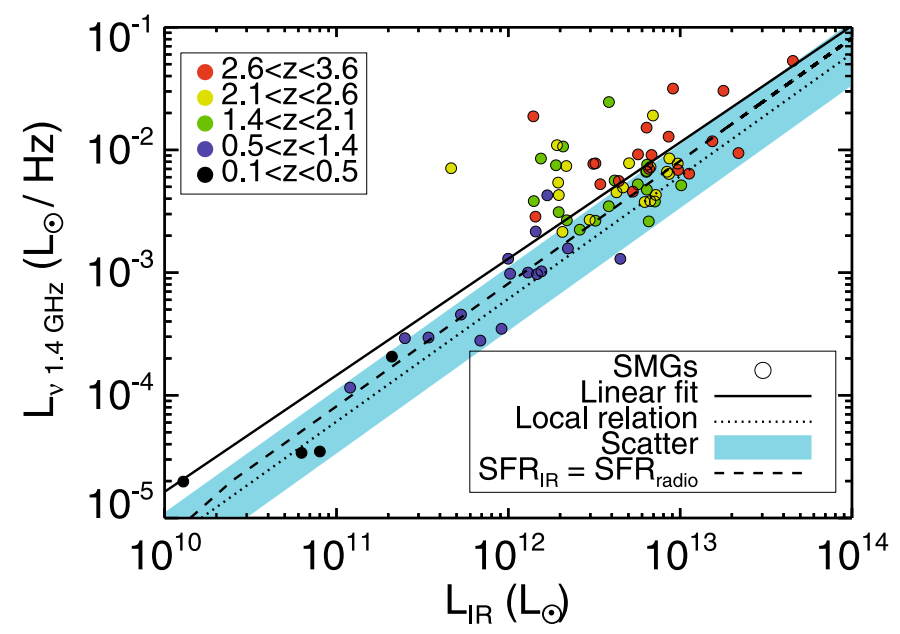

Fig. 5. Radio luminosity density as a function of infrared $(8-1000 \mu \mathrm{m})$ luminosity of SMGs showing a linear relation, though with a normalization offset from the local relation by a factor of $\sim 2.1$ towards higher radio luminosities (Sect. 5.4.1). Circles: values for individual SMGs color-coded by redshift. Solid line: linear fit to the data (Eq. (1)). Dotted line: the mean local relation (Bell 2003). Shaded area: its scatter. Dashed line: the track where SFR IR (Kennicutt 1998) is equal to $\mathrm{SFR}_{\text {radio }}$ (Bell 2003). The strong outliers (above the line) at highluminosity end are probably caused by AGN activity increasing radio luminosities.

\subsubsection{Stellar masses}

SMGs having stellar masses of $\sim 10^{11}-10^{12} M_{\odot}$ (Col. 8 of Table A.3 and Fig. 2) are among the most massive galaxies in the Universe, regardless of redshift (compare with Figs. 2 and 4 of Castro Cerón et al. 2006, 2009, respectively). This property makes them natural candidates for the progenitors of the presentday ellipticals.

The relatively tight range of stellar masses is likely not a result of sensitivity limits at optical and near-IR. This is because i) galaxies with stellar mass as low as $\sim 10^{9} M_{\odot}$ would have been detected in deep Spitzer imaging at redshifts $z \sim 2$ (e.g. Reddy et al. 2006); ii) our sample accounts for $50 \%$ of the parent Chapman et al. (2005) sample (and only $30 \%$ of the parent sample may have different properties than our sample, see Sect. 2), so it is unlikely that we miss only the low-mass objects. Therefore, high $M_{*}$ seems to be an intrinsic property of submillimeter-selected galaxies. Mergers of less massive galaxies could not result in a powerful starburst giving rise to detectable submillimeter emission (see also Davé et al. 2010).

Only a minor part (median $\sim 8 \%$, Col. 9 of Table A.3 and Fig. 2) of the stellar populations present in SMGs has been formed during the ongoing starburst episodes. Hence, even though SMGs probably evolve into ellipticals, the majority of the stellar mass in such ellipticals had been created before the submillimeter-bright phase.

This could mean that the current SFRs and stellar masses of SMGs are only loosely connected and indeed this manifests itself in a very high spread (around two orders of magnitude) in SSFRs in our sample even though the stellar mass range is relatively tight: $\sim 10^{11}-10^{12} M_{\odot}$ (Fig. 2). This behaviour is unusual compared to other galaxies (see Castro Cerón et al. 2006, 2009).

However we note that the low stellar masses created in the ongoing starburst may partially be an effect of the assumed starburst ages of 50 Myr. If a starburst duration of 100-200 Myr were adopted (Smail et al. 2004; Borys et al. 2005; Hainline 2008; Tacconi et al. 2008) the resulting $M_{\text {burst }}$ could be higher by a factor of $\sim 2-4$.

The mass-to-light ratios, $M_{*} / L_{K}$, of SMGs (Col. 10 of Table A.3 and Fig. 2) are typical for massive galaxies. Specifically, the median $\left(0.68 M_{\odot} L_{\odot}^{-1}\right)$ is similar to the values for $M_{*}>10^{11} M_{\odot}$ galaxies (Drory et al. 2004, their Table 1) and to simulated massive galaxies at $z \sim 1$ (Courty et al. 2007, their Fig. 4).

\subsubsection{Dust properties}

Our fits suggest that SMGs are moderately dust-obscured with a median $A_{V} \sim 2$ mag (Col. 14 of Table A.3). Our estimates are consistent within 1-2 $\sigma$ with the mean/median values obtained by Smail et al. (2004, 1.70-2.44), Swinbank et al. (2004, $3.0 \pm$ 1.0), Borys et al. $(2005,1.7 \pm 0.2)$ and Hainline $(2008,1.7 \pm$ 0.1 ) based on near-IR data. For individual SMGs we obtained systematically larger extinction (median difference of $\sim 0.3 \mathrm{mag}$ ) than Hainline (2008). The difference may be accounted for if there is significant extinction even in Spitzer IRAC data.

The dust density of SMGs at $z<0.5$ (Col. 9 of Table 1 ) is approximately $3 \%$ of the total local $(0.013<z<0.18)$ dust budget of $\log \rho_{\text {dust }}=5.57_{-0.17}^{+0.12} M_{\odot} \mathrm{Mpc}^{-3}$ given by Driver et al. (2007) based on an assumed dust-to-light ratio. Therefore SMGs contribute very little to the dust budget at low redshifts.

In our sample of SMGs $\rho_{\text {dust }}$ does not change significantly from $z \sim 3.6$ to $z \sim 0.5$. We do not detect any evolution of dust mass in SMGs across the entire redshift range (Fig. 2). A constant dust mass density across redshifts $0-3.5$ was also found by Pascale et al. (2009) based on a stacking analysis at submillimeter wavelengths of galaxies selected at $24 \mu \mathrm{m}$.

The question is what happened to the dust produced in SMGs. If they evolve into dust-poor ellipticals, then the dust is not simply stored in their end-products (as is probably the case for stellar masses). It is therefore plausible that dust is either blown away (by stellar and/or AGN winds) or absorbed in star formation, or destroyed during subsequent evolution after the SMG event.

\subsubsection{Comparison with GRB hosts}

In Michałowski et al. (2008) we presented a hypothesis that gamma-ray burst (GRB) host galaxies may constitute a subsample of hotter/less luminous counterparts of SMGs. Indeed, the UV-to-IR SEDs of three $z \sim 2-3$ SMGs $^{8}$ are consistent with $z \sim 1$ submillimeter/radio bright GRB hosts (dashed lines in Fig. A.1 from Michałowski et al. 2008), but 1.2-3.9 times more luminous. These three SMGs are similar to GRB hosts with respect to their hot dust temperatures ( 40-60 K), high SSFRs $\left(\gtrsim 2 \mathrm{Gyr}^{-1}\right.$, high fraction of stellar mass formed in the ongoing starburst $(>10 \%)$ and blue optical colors.

If larger samples of GRB hosts shows a similar tendency that their brightest members overlap with the hotter subsample of SMGs, then GRB events will provide an effective way of selecting hot SMGs, otherwise difficult to localize.

\footnotetext{
8 SMMJ141750.50+523101.0, SMMJ141802.87+523011.1, SMMJ163627.94+405811.2
} 


\subsection{Contribution to stellar mass assembly}

\subsubsection{Star formation rate volume density}

SFR densities of SMGs were calculated as described in Sect. 3.2. In order to assess the accuracy of our simplified method of dividing the sum of the SFRs of the detected SMGs by the total survey volume, we compare our estimates with those resulting from detailed calculation of the volume contribution of individual SMGs done by Chapman et al. (2005, based on the same sample as we analyse) and Wall et al. (2008, based on 35 SMGs in GOODS-N field of which 17 have spectroscopic redshifts). The comparison is shown in Fig. 4. Our results in two high-redshift bins $(z>2)$ corrected for incompleteness (Sect. 3.2) are consistent with that of Chapman et al. (2005) and Wall et al. (2008). At lower redshifts we find values similar to Chapman et al. (2005), but an order of magnitude lower than Wall et al. (2008). Therefore we conclude that i) our method to calculate volumes is accurate, since it gives consistent results with other estimates; and ii) our sample is incomplete in the three low-redshift bins as anticipated in Sect. 2.

From Fig. 4 (and Cols. 4 and 5 of Table 1) it is apparent that a $\rho_{\text {SFR }}$ of SMGs starts to decline (with cosmic time) earlier ( about $z \sim 2)$ than that of other galaxies $(z \sim 1)$. More quantitatively, SMGs harbour $\sim 20 \%$ of the cosmic $\rho_{\text {SFR }}$ at $z \sim 2-3.6$ (Col. 5), but their contribution drops to $\sim 9 \%$ at $0.5<z<1.4$. It is likely that at lower redshifts, due to the decreased rate of mergers (e.g. Rawat et al. 2008; de Ravel et al. 2009), there are fewer galaxies left that can still sustain high SFRs to be detected at submillimeter wavelengths. However, part of the decrease of SMG $\rho_{\text {SFR }}$ can be explained by the "redshift desert", which makes it difficult to measure redshifts of $z \sim 1.2-1.8$ SMGs (see Sect. 2).

A high value of $\rho_{\mathrm{SFR}}$ of SMGs at $z \sim 2-3$ and the subsequent decline are consistent with the hypothesis that the SMG population is a manifestation of powerful starburst episodes evolving into the present-day ellipticals (as discussed in Sect. 5.2.2). In this scenario galaxies detected in the submillimeter at high- $z$ do not enter the sample of SMGs at low- $z$ because they have already evolved into passive galaxies. It has indeed been found that ellipticals contain old stars formed at $z \sim 1.5-4$ (Daddi et al. 2000; van Dokkum \& Franx 2001; van de Ven et al. 2003). The evolution of SMGs into ellipticals has also been claimed by several authors based on their luminosity function (Smail et al. 2004), huge luminosities (Eales et al. 1999) and gas reservoirs (Smail et al. 2002; Greve et al. 2005), strong clustering (Ivison et al. 2000; Almaini et al. 2003), space density and morphology (Barger et al. 1999; Lilly et al. 1999; Trentham et al. 1999; Swinbank et al. 2006) and evolutionary SED models (Takagi et al. 2004).

Knudsen et al. (2008b) analysed number counts of SMGs fainter than the SCUBA confusion limit, using those behind clusters of galaxies magnified by lensing. They concluded that the integrated light produced by the SMGs brighter than $0.1 \mathrm{mJy}$ (i.e. LIRGs and ULIRGs with roughly $L_{\mathrm{IR}}>8 \times 10^{10} L_{\odot}$ and SFR $>15 M_{\odot} \mathrm{yr}^{-1}$ ) is comparable to the extragalactic background light (EBL) at $850 \mu \mathrm{m}$ (see also Blain et al. 1999; Cowie et al. 2002). This means that these galaxies host the majority of the cosmic obscured star formation. Knudsen et al. (2008b) also found that sources brighter than $2.5 \mathrm{mJy}$ (roughly the limit of the survey considered here) contribute $\sim 25 \%$ to the to EBL at $850 \mu \mathrm{m}$ (see also Hughes et al. 1998; Barger et al. 1999; Wang et al. 2004; Coppin et al. 2006). Together with our results this implies that as much as $\sim 80 \%(4 \times 20 \%)$ of the cosmic star formation at $z \sim 2-3.6$ reside in SMGs brighter than $0.1 \mathrm{mJy}$. This is only true if the faint ( $<2 \mathrm{mJy}$ ) SMGs have similar dust temperatures to the brighter ones. If they are colder (hotter) their submillimeter fluxes corresponds to lower (higher) SFRs (because it is calibrated to total IR emission) and therefore the total SMG population contribute less (more) than $80 \%$ to the cosmic $\rho_{\text {SFR }}$. This picture is however complicated, because based on stacking analysis it has been claimed that the distribution of the faint SMGs peaks at lower redshifts $(z<1.5$; Wang et al. 2006; Serjeant et al. 2008).

Our overall conclusion is that the SMG population plays a significant role at redshifts $z \sim 2-4$, namely sources brighter than $\sim 3(0.1) \mathrm{mJy}$ at $850 \mu \mathrm{m}$ host $20 \%$ (80\%) of cosmic star formation. Their contribution can however be lower in reality if very small (but numerous) galaxies are missed in all high- $z$ flux-limited galaxy surveys. In such a case the total SFR density (color points in Fig. 4) would be underestimated. To solve this issue much deeper surveys at high- $z$ are necessary, either blankfield or for well-selected dwarf galaxy samples (e.g., GRB hosts or Ly $\alpha$ emitters).

Zheng et al. (2007) estimated $\rho_{\mathrm{SFR}}$ at $z \sim 0.9$ for massive galaxies $\left(M_{*}>10^{11} M_{\odot}\right)$ down to $R<24$ mag (only $\sim 40 \%$ of SMGs satisfy the latter criterion) equal to $0.0052_{-0.0021}^{+0.0020} M_{\odot} \mathrm{yr}^{-1} \mathrm{Mpc}^{-3}$. This value is only a factor of 2 lower than our estimate for the SMGs at $0.5<z<1.4$ (Table 1). Therefore, although SMGs do not host a major fraction of the cosmic SFR at these redshifts, they contribute significantly $(0.0102 /(0.0052 \times 0.6+0.0102) \sim 66 \%)$ to the SFR budget of massive galaxies.

\subsubsection{Stellar mass volume density}

Stellar mass densities of SMGs were calculated as described in Sect. 3.2. Figure 4 and Table 1 (Cols. 6 and 7) show that at $z \sim 2-3.6$ a significant part ( $\sim 30-50 \%)$ of the cosmic stellar mass had been formed in the progenitors of SMGs. At lower redshifts $\rho_{*}$ of SMGs (and hence their contribution to the cosmic $\rho_{*}$ ) drops, likely because the majority of SMGs at higher redshifts had already evolved into passive galaxies at $z \sim 1.5$, and so dropped out of our submillimeter-selected sample. Moreover the sample is incomplete at $z \sim 1.2-1.8$ due to the "redshift desert" (see Sect. 2). This brings down the densities of SMGs in the low$z$ bins.

Since most of the stellar mass of SMGs has not been formed in the ongoing starburst (Sect. 5.2.2), their $\rho_{*}$ reflects the integrated contribution of SMGs to the cosmic $\rho_{\mathrm{SFR}}$. Therefore the relatively high contribution of SMGs to the cosmic $\rho_{*}$ in the last redshift bin $(\sim 31 \%$, Col. 7 of Table 1$)$ means that SMGs play a non-negligible role in the cosmic stellar assembly even at $z>3.6$. This can be tested by analysis of a sample of $z \gtrsim 4$ SMGs in a defined survey sky area (e.g. Michałowski et al. 2010; Younger et al. 2009a, note that these results are likely affected by cosmic variance). It has been confirmed that such distant SMGs exist (Capak et al. 2008; Knudsen et al. 2008a, 2010; Schinnerer et al. 2008; Coppin et al. 2009; Daddi et al. 2009b,a).

\subsection{Source of emission}

\subsubsection{IR-radio correlation}

With our full SED modelling of 76 SMGs we confirm the results of Hainline (2008) on the correlation between IR and radio luminosities. Figure 5 shows that SMGs follow a linear IR-radio correlation. The two outliers (with $q \sim 1.3$, see Sect. 5.4.2) are 
probably caused by AGN activity contributing significantly to radio luminosities. A linear fit gives:

$$
\begin{aligned}
\log \left(L_{v 1.4 \mathrm{GHz}} / L_{\odot} \mathrm{Hz}^{-1}\right)= & (0.95 \pm 0.07) \log \left(L_{\mathrm{IR}} / L_{\odot}\right) \\
& -(14.3 \pm 0.8) .
\end{aligned}
$$

The slope is consistent (within errors) with unity, suggestive of the linear relation between $L_{v 1.4 \mathrm{GHz}}$ and $L_{\mathrm{IR}}$ at the high-end $\left(L_{\mathrm{IR}} \gtrsim 10^{11} L_{\odot}\right)$ of the galaxy luminosity function (a similar value of $1.064 \pm 0.025$ was found by Hainline 2008).

The IR-radio correlation is usually quantified by the ratio of IR and radio luminosities, $q$ (see Sect. 3.1). The mean $q$ for SMGs $(2.32 \pm 0.04$, scatter: 0.34$)$ is significantly lower than that of local star-forming galaxies (2.64 with a scatter of 0.26 ; Bell 2003). Similar offsets were reported by Kovács et al. (2006), Murphy et al. (2009) and Murphy (2009) based on smaller samples of SMGs. We conclude that at $z>1.4$ SMGs have radio luminosities on average a factor of $\sim 2.1$ larger $(\Delta q \sim-0.32)$ than what would result from the local relation. The difference is significant at the level of $4-5 \sigma$ and can be explained in three ways.

Radio-loud AGNs have on average low $q$ values (see e.g. Miller \& Owen 2001; Yun et al. 2001; Yang et al. 2007). If $\gtrsim 50 \%$ of the radio emission of SMGs is powered by AGNs, then the radio luminosities of SMGs higher by a factor of $\sim 2.1$ can be accounted for. However, there are indications that SMGs are starburst-dominated (see Sect. 5.4.2), so we deem this explanation less likely.

Another explanation is that the radio excess is a result of the bias against radio-faint sources in our sample (see Sect. 2). This can be tested when a sample of SMGs with localizations (and hence redshifts) independent of radio detections is available (e.g. Daddi et al. 2009b,a; Knudsen et al. 2010; Weiß et al. 2009a).

The third possibility is that some properties influencing the IR or radio emission are intrinsically different for SMGs and local galaxies. The sample of Bell (2003) includes local normal, star-forming spiral and irregular galaxies, blue compact dwarfs, starburst galaxies and ULIRGs. Therefore the difference in the properties between this sample and such extreme galaxies as SMGs is expected. Such explanation was offered by Lacki et al. (2009) and Lacki \& Thompson (2009). Their numerical modelling showed that cosmic-ray electrons in "puffy starbursts" (vertically and radially extended galaxies with vertical scale heights $\sim 1 \mathrm{kpc}$ ) experience weaker bremsstrahlung and ionization losses resulting in stronger radio emission. Indeed, there are indications that SMGs are extended on vertical scales of $\sim 1$ kpc (Lacki \& Thompson 2009; Tacconi et al. 2006, 2008; Genzel et al. 2008; Younger et al. 2008; Law et al. 2009), so we find this explanation probable.

The systematic uncertainties in the determination of $L_{\mathrm{IR}}$ (factor of $\lesssim 2$, Sect. 4) may in principle also explain the offset. However, we find this unlikely because similar offsets were found by other authors using different fitting methods (Kovács et al. 2006; Murphy et al. 2009; Murphy 2009).

The $q$ values for SMGs are shown in Fig. 6 as a function of redshift. We do not detect any significant evolution across the redshift range 1.4-3.6. The only sign of evolution is that the mean $q$ in the low-redshift bin $(0.5<z<1.4)$ is above the value found at higher redshifts $(\sim 4 \sigma)$. This can be explained either by the contribution of reprocessed emission from low-mass stars (cirrus emission, e.g. Yun et al. 2001, and references therein) to the IR, or by the fact that at low redshifts SMGs are more similar to other local galaxies and do not exhibit large vertical scale heights characteristic for "puffy starbursts" (see above).

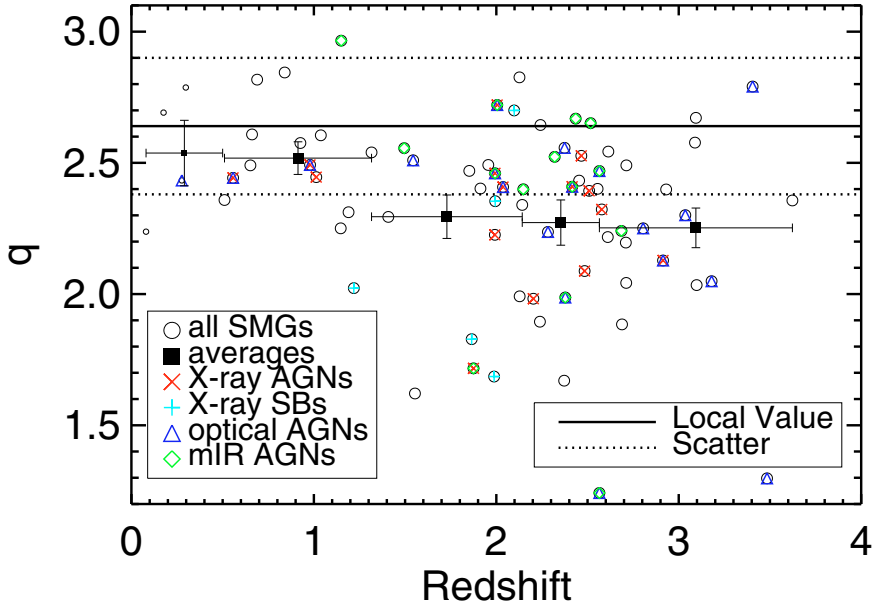

Fig. 6. The ratio of the infrared $(8-1000 \mu \mathrm{m})$ and radio luminosities $q$ (defined in Sect. 3.1) as a function of redshift of SMGs. It provides evidence that a linear IR-radio correlation holds for SMGs up to $z \sim 3.6$, though with a normalization offset from the local relation by a factor of $\sim 2.1(\Delta q \sim-0.32)$ towards higher radio luminosities (Sect. 5.4.1). Circles: values for individual SMGs. Squares: the mean values (and errors on the mean) in five redshift bins containing equal number of galaxies (Table 1 and Sect. 3.2). Small symbols indicate $z<0.5$ objects. Red crosses: SMGs classified as AGNs based on X-ray emission (Alexander et al. 2005). Light blue plus signs: SMGs classified as starbursts based on X-ray emission (Alexander et al. 2005). Violet triangles: SMGs classified as AGNs based on optical spectra (Chapman et al. 2005). Green diamonds: SMGs classified as AGNs based on a mid-IR power-law (Sect. 5.4.2). The mean local $q=2.64$ (Bell 2003) is shown as a solid line with 0.26 scatter (dotted lines). The $q$ values for majority of AGN-classified SMGs do not differ from the rest of the SMG population (see Sect. 5.4.2).

It is important to note that the derived linear IR-radio correlation for SMGs is not a consequence of the use of the SED templates (which were tuned to fulfill this correlation locally), because the radio luminosities used here were derived based on the observational data only, independent of the SED modeling.

\subsubsection{AGN activity}

As discussed in Sect. 5.4.1, AGN activity could explain low $q$ values of SMGs. This is at least true for the two SMGs with lowest $q^{9}$, spectroscopically classified as AGN (Chapman et al. 2005).

In the SEDs of SMGs there are clear signs that some of them host AGNs (though, not necessarily a bolometrically dominant ones). Radio datapoints are higher than model predictions by more than $3 \sigma$ in $36 \%$ (27/76) of SMGs, whereas they are lower than models only for $8 \%(6 / 76)$. This may hint at an AGN contribution in these galaxies. However, 4 out of $5 \mathrm{X}$-ray identified starbursts (Col. 16 of Table A.3) also exhibit radio excess, so we find other explanations of radio excess presented in Sect. 5.4.1 more reliable.

Another indication of an AGN contribution is that $18 \%$ (14/76) of SMGs show a mid-IR power-law AGN feature incompatible with our starburst models (see Fig. A.1 and Col. 16 of Table A.3). However, rest-frame $2-5 \mu \mathrm{m}$ excess was also interpreted as a tracer of recent star formation (Mentuch et al. 2009).

\footnotetext{
${ }^{9}$ SMMJ131215.27+423900.9, SMMJ141813.54+522923.4
} 
Finally, three SMGs ${ }^{10}$ have exceptionally high SFR $_{U V}$ (>500 $M_{\odot} \mathrm{yr}^{-1}$, Col. 4 of Table A.3). Strikingly, all of them were fitted with non-starburst models ( $M_{\text {burst }}=0$, Col. 9), so modeling is consistent with these high SFRs being continuous (the same is true for three other non-starburst SMGs with high $\mathrm{SFR}_{\mathrm{IR}}$ ). Such a scenario is unlikely, so this hints at an AGN contribution to the UV/IR emission.

However, the fact that we obtained reasonable SED fits for most of the SMGs using purely star-forming models (Fig. A.1) hints at the conclusion that AGN activity is not dominant in our sample.

We investigated the issue of AGN activity further by analysing the average $q$ values of the following subsamples (see also Fig. 6): X-ray identified (Alexander et al. 2005) AGNs: $2.32 \pm 0.06$ and starbursts: $2.12 \pm 0.18$; optically identified AGNs (Chapman et al. 2005): $2.27 \pm 0.09$; and mid-IR identified AGNs (see above): $2.36 \pm 0.12$. All subsamples are consistent with the value derived for the entire sample (2.32) Hence, we confirm the finding of Hainline (2008) that even the AGN-classified SMGs follow a linear IR-radio correlation. This means that even if an AGN is present it does not contribute to the emission of an SMG significantly (with the exception of the two $q \sim 1.3$ sources).

This is in line with i) the X-ray studies of SMGs indicating that the contribution of AGN activity to their IR emission is only $\sim 8 \%$ on average (Alexander et al. 2005); ii) midIR colors of SMGs indicating that AGNs dominate the emission at these wavelengths only in 13-19\% cases (Hainline et al. 2009); iii) mid-IR spectroscopy of SMGs revealing only weak AGN-like continua (Valiante et al. 2007; Pope et al. 2008; Menéndez-Delmestre et al. 2007; Menéndez-Delmestre et al. 2009; Murphy et al. 2009; Watabe et al. 2009); iv) near-IR spectroscopy revealing that starbursts dominate the emission of SMGs (Swinbank et al. 2004). Moreover, de Vries et al. (2007) found that star formation processes (if present) account for at least $75 \%$ of the radio luminosities of optically-selected AGNs.

Therefore we conclude that AGNs are present in a significant fraction of SMGs, but their contribution to the IR emission is at most minor.

\subsection{Comparison of our results with the literature}

For the sample of SMGs discussed in this paper there are previous estimates of some of their properties. In this section we compare them with our results.

Chapman et al. (2005) derived $L_{\mathrm{IR}}$ and $T_{\mathrm{d}}$ based only on the $850 \mu \mathrm{m}$ and $1.4 \mathrm{GHz}$ data. There is no systematic difference between the determinations of $T_{\mathrm{d}}$ (our median of $38.7 \mathrm{~K}$, theirs: $38.3 \mathrm{~K}$ ). The mean difference between individual datapoints is $4 \mathrm{~K}(\sim 10 \%)$. However, our values for $L_{\mathrm{IR}}$ are systematically lower than theirs (the median ratio of individual datapoints is 1.7). We find our values more reliable since they are based on data spanning a wider wavelength range. Overestimation of $L_{\mathrm{IR}}$ when using only $850 \mu \mathrm{m}$ and $1.4 \mathrm{GHz}$ was also noticed by Kovács et al. (2006) and Pope et al. (2006).

Kovács et al. (2006) investigated a subsample observed at $350 \mu \mathrm{m}$. Their median dust mass $\left(9.04 \log M_{\odot}\right)$ and $q$ value (2.20) are consistent with our estimates (9.01 and 2.35, respectively). The median difference between individual datapoints is $\sim 30 \%$ for dust masses and $\sim 13 \%$ for $q$.

The median stellar mass for a subsample of 13 SMGs investigated by Borys et al. $\left(2005,11.51 \log M_{\odot}\right)$ is close to our

\footnotetext{
10 SMMJ123716.01+620323.3, SMMJ131215.27+423900.9, SMMJ131222.35+423814.1
}

value (11.70). However, estimates of Hainline (2008, median $10.82 \log M_{\odot}$ ) for 64 SMGs are a factor of $\sim 5.6$ smaller than our values (11.57). Hainline (2008) postulated that the discrepancy between her results and those of Borys et al. (2005) arose from a combination of systematic differences between the applied SED models and a higher AGN contribution in the $K$-band (used by Borys et al. 2005) with respect to the $H$-band. Our estimates are based on all the available photometric data, and so we find the former explanation more likely. In particular, the differences in the applied stellar population models and their ages may explain the discrepancy.

\section{Conclusions}

We have investigated the UV-to-radio SEDs of 76 SMGs $\left(S_{850} \gtrsim\right.$ $3 \mathrm{mJy}$ ) with spectroscopic redshifts (0.080-3.623). For the first time the properties of such a significant sample has been derived consistently using all available data. The resulting SFRs (median $\left.713 M_{\odot} \mathrm{yr}^{-1}\right)$ and stellar masses $\left(11.57 \log M_{\odot}\right)$ are among the highest in the Universe.

Such high stellar masses, already present at redshifts $\sim 2-3$, require that SMGs experienced either at least two starburst episodes, or a merger of several smaller galaxies. Our modeling suggests that only a minor fraction $(8 \%)$ of their stellar populations was formed during the ongoing starburst episodes. This is supported by the fact that the SFRs and $M_{*}$ of SMGs are basically disconnected, i.e. we observe two orders of magnitude spread in SSFRs whereas the range of $M_{*}$ is relatively narrow: $10^{11}-10^{12} M_{\odot}$. We concluded that dust is blown away or destroyed during the evolution of SMGs, since it is not stored in the likely end-products of SMGs, elliptical galaxies.

Indeed, the high stellar masses and the evolution of the SFR and stellar mass densities of SMGs are consistent with a scenario in which SMGs are progenitors of present-day ellipticals.

We found that SMGs contribute significantly to the cosmic SFR, $\rho_{\text {SFR }}(\sim 20 \%)$ and stellar mass, $\rho_{*}(30-50 \%)$ densities at $z \sim 2-4$. If we consider submillimeter sources down to $0.1 \mathrm{mJy}$ the contribution to $\rho_{\mathrm{SFR}}$ rises to $\sim 80 \%$.

Our analysis suggests that a linear IR-radio correlation holds for SMGs at least up to a redshift of 3.6, but they are $\sim 2.1$ times brighter at radio wavelengths than what would result from the local correlation.

Acknowledgements. We thank Joanna Baradziej, José María Castro Cerón, Thomas Greve, Brian Lacki, Kim Nilsson, Jesper Sommer-Larsen, Sune Toft, Bärbel Tress and Gunther Tress for discussion and comments; our referee for help with improving this paper; Scott Chapman for information on the survey areas; Jorge Iglesias-Páramo for kindly providing his SED templates; and Fabio Fontanot for kindly providing his model and data on star formation density. We acknowledge use of the extensive Spitzer database in the $\mathrm{PhD}$ thesis of Laura Hainline.

M. J. M. would like to acknowledge support from The Faculty of Science, University of Copenhagen. The Dark Cosmology Centre is funded by the Danish National Research Foundation. This research has made use of NASA's Astrophysics Data System Bibliographic Services.

\section{References}

Ajiki, M., Taniguchi, Y., Fujita, S., et al. 2003, AJ, 126, 2091 Alexander, D. M., Bauer, F. E., Chapman, S. C., et al. 2005, ApJ, 632, 736 Alexander, D. M., Brandt, W. N., Smail, I., et al. 2008, AJ, 135, 1968 Almaini, O., Scott, S. E., Dunlop, J. S., et al. 2003, MNRAS, 338, 303 Appleton, P. N., Fadda, D. T., Marleau, F. R., et al. 2004, ApJS, 154, 147 Aravena, M., Bertoldi, F., Carilli, C., et al. 2010, ApJ, 708, L36 Arnouts, S., Walcher, C. J., Le Fèvre, O., et al. 2007, A\&A, 476, 137 Ashby, M. L. N., Dye, S., Huang, J.-S., et al. 2006, ApJ, 644, 778 Austermann, J. E., Aretxaga, I., Hughes, D. H., et al. 2009, MNRAS, 393, 1573 
Barger, A. J., Cowie, L. L., \& Sanders, D. B. 1999, ApJ, 518, L5 Barger, A. J., Cowie, L. L., \& Richards, E. A. 2000, AJ, 119, 2092 Bell, E. F. 2003, ApJ, 586, 794

Bell, E. F., McIntosh, D. H., Katz, N., et al. 2003, ApJS, 149, 289 Bell, E. F., Zheng, X. Z., Papovich, C., et al. 2007, ApJ, 663, 834

Berciano Alba, A., Koopmans, L. V. E., Garrett, M. A., Wucknitz, O., \& Limousin, M. 2010, A\&A, 509, A54

Beswick, R. J., Muxlow, T. W. B., Thrall, H., Richards, A. M. S., \& Garrington, S. T. 2008, MNRAS, 385, 1143

Blain, A. W. 1997, MNRAS, 290, 553

Blain, A. W., \& Longair, M. S. 1996, MNRAS, 279, 847

Blain, A. W., Kneib, J., Ivison, R. J., et al. 1999, ApJ, 512, L87

Blain, A. W., Smail, I., Ivison, R. J., Kneib, J. P., \& Frayer, D. T. 2002, Phys. Rep., 369, 111

Blain, A. W., Chapman, S. C., Smail, I., et al. 2004, ApJ, 611, 52

Borch, A., Meisenheimer, K., Bell, E. F., et al. 2006, A\&A, 453, 869

Borys, C., Smail, I., Chapman, S. C., et al. 2005, ApJ, 635, 853

Bouwens, R., Broadhurst, T., \& Illingworth, G. 2003a, ApJ, 593, 640

Bouwens, R. J., Illingworth, G. D., Rosati, P., et al. 2003b, ApJ, 595, 589

Bouwens, R. J., Illingworth, G. D., Thompson, R. I., et al. 2004, ApJ, 606, L25

Bouwens, R. J., Illingworth, G. D., Blakeslee, J. P., et al. 2006, ApJ, 653, 53

Bouwens, R. J., Illingworth, G. D., Franx, M., et al. 2007, ApJ, 670, 928

Boyle, B. J., Cornwell, T. J., Middelberg, E., et al. 2007, MNRAS, 376, 1182

Brinchmann, J., \& Ellis, R. S. 2000, ApJ, 536, L77

Brinchmann, J., Charlot, S., White, S. D. M., et al. 2004, MNRAS, 351, 1151

Bundy, K., Ellis, R. S., Conselice, C. J., et al. 2006, ApJ, 651, 120

Bunker, A. J., Stanway, E. R., Ellis, R. S., et al. 2004, MNRAS, 355, 374

Capak, P., Cowie, L. L., Hu, E. M., et al. 2004, AJ, 127, 180

Capak, P., Carilli, C. L., Lee, N., et al. 2008, ApJ, 681, L53

Caputi, K. I., McLure, R. J., Dunlop, J. S., Cirasuolo, M., \& Schael, A. M. 2006, MNRAS, 366, 609

Caputi, K. I., Lagache, G., Yan, Lin, et al. 2007, ApJ, 660, 97

Castro Cerón, J. M., Michałowski, M., Hjorth, J., et al. 2006, ApJ, 653, L85

Castro Cerón, J. M., Michałowski, M. J., Hjorth, J., et al. 2009, ApJ, submitted, [arXiv:0803.2235v1]

Chapman, S. C., Richards, E. A., Lewis, G. F., Wilson, G., \& Barger, A. J. 2001, ApJ, 548, L147

Chapman, S. C., Barger, A. J., Cowie, L. L., et al. 2003a, ApJ, 585, 57

Chapman, S. C., Windhorst, R., Odewahn, S., Yan, H., \& Conselice, C. 2003b, ApJ, 599, 92

Chapman, S. C., Smail, I., Windhorst, R., Muxlow, T., \& Ivison, R. J. 2004, ApJ, 611,732

Chapman, S. C., Blain, A. W., Smail, I., et al. 2005, ApJ, 622, 772

Clements, D., Eales, S., Wojciechowski, K., et al. 2004, MNRAS, 351, 447

Clements, D. L., Vaccari, M., Babbedge, T., et al. 2008, MNRAS, 387, 247

Cohen, J. G. 2002, ApJ, 567, 672

Cole, S., Norberg, P., Baugh, C. M., et al. 2001, MNRAS, 326, 255

Condon, J. J. 1989, ApJ, 338, 13

Condon, J. J. 1992, ARA\&A, 30, 575

Condon, J. J., Cotton, W. D., \& Broderick, J. J. 2002, AJ, 124, 675

Connolly, A. J., Szalay, A. S., Dickinson, M., Subbarao, M. U., \& Brunner, R. J. 1997, ApJ, 486, L11

Conselice, C. J., Blackburne, J. A., \& Papovich, C. 2005, ApJ, 620, 564

Coppin, K., Chapin, E. L., Mortier, A. M. J., et al. 2006, MNRAS, 372, 1621

Coppin K., Halpern, M., \& Scott, D. 2008, MNRAS, 384, 1597

Coppin, K. E. K., Smail, I., Alexander, D. M., et al. 2009, MNRAS, 395, 1905

Courty, S., Björnsson, G., \& Gudmundsson, E. H. 2007, MNRAS, 376, 1375

Cowie, L. L., \& Hu, E. M. 1998, AJ, 115, 1319

Cowie, L. L., Songaila, A., Hu, E. M., et al. 1996, AJ, 112, 839

Cowie, L. L., Songaila, A., \& Barger, A. J. 1999, AJ, 118, 603

Cowie, L. L., Barger, A. J., \& Kneib, J. 2002, AJ, 123, 2197

Daddi, E., Cimatti, A., \& Renzini, A. 2000, A\&A, 362, L45

Daddi, E., Dannerbauer, H., Krips, M., et al. 2009a, ApJ, 695, L176

Daddi, E., Dannerbauer, H., Stern, D., et al. 2009b, ApJ, 694, 1517

Dahlen, T., Mobasher, B., Dickinson, M., et al. 2007, ApJ, 654, 172

Davé, R., Finlator, K., Oppenheimer, B. D., et al. 2010, MNRAS, 404, 1355

de Ravel, L., Le Fèvre, O., Tresse, L., et al. 2009, A\&A, 498, 379

de Vries, W. H., Hodge, J. A., Becker, R. H., White, R. L., \& Helfand, D. J. 2007, AJ, 134, 457

Devlin, M. J., Ade, P. A. R., Aretxaga, I., et al. 2009, Nature, 458, 737

Dickinson, M., Papovich, C., Ferguson, H. C., et al. 2003, ApJ, 587, 25

Driver, S. P., Allen, P. D., Graham, A. W., et al. 2006, MNRAS, 368, 414

Driver, S. P., Popescu, C. C., Tuffs, R. J., et al. 2007, MNRAS, 379, 1022

Drory, N., Bender, R., Feulner, G., et al. 2004, ApJ, 608, 742

Drory, N., Salvato, M., Gabasch, A., et al. 2005, ApJ, 619, L131

Dunne, L., Ivison, R. J., Maddox, S., et al. 2009, MNRAS, 394, 3

Dye, S., Eales, S. A., Aretxaga, I., et al. 2008, MNRAS, 386, 1107

Dye, S., Ade, P. A. R., Bock, J. J., et al. 2009, ApJ, 703, 285
Eales, S., Lilly, S., Gear, W, et al. 1999, ApJ, 515, 518

Eales, S., Chapin, E. L., Devlin, M. J., et al. 2009, ApJ, 707, 1779

Egami, E., Dole, H., \& Huang, J.-S. 2004, ApJS, 154, 130

Elsner, F., Feulner, G., \& Hopp, U. 2008, A\&A, 477, 503

Erb, D. K., Steidel, C. C., Shapley, A. E., et al. 2006, ApJ, 646, 107

Eyles, L. P., Bunker, A. J., Ellis, R. S., et al. 2007, MNRAS, 374, 910

Flores, H., Hammer, F., Thuan, T. X., et al. 1999, ApJ, 517, 148

Fomalont, E. B., Kellermann, K. I., Cowie, L. L., et al. 2006, ApJS, 167, 103

Fontana, A., Donnarumma, I., Vanzella, E., et al. 2003, ApJ, 594, L9

Fontana, A., Pozzetti, L., Donnarumma, I., et al. 2004, A\&A, 424, 23

Fontana, A., Salimbeni, S., Grazian, A., et al. 2006, A\&A, 459, 745

Franceschini, A., Rodighiero, G., Cassata, P., et al. 2006, A\&A, 453, 397

Frayer, D. T., Chapman, S. C., Yan, L., et al. 2004, ApJS, 154, 137

Fujita, S. S., Ajiki, M., Shioya, Y., et al. 2003a, AJ, 125, 13

Fujita, S. S., Ajiki, M., Shioya, Y., et al. 2003b, ApJ, 586, L115

Gallego, J., Zamorano, J., Aragon-Salamanca, A., et al. 1995, ApJ, 455, L1

Gallego, J., García-Dabó, C. E., Zamorano, J., Aragón-Salamanca, A., \& Rego,

M. 2002, ApJ, 570, L1

Garn, T., Green, D. A., Riley, J. M., et al. 2009, MNRAS, 397, 1101

Garrett, M. A. 2002, A\&A, 384, L19

Geach, J. E., Smail, I., Best, P. N., et al. 2008, MNRAS, 388, 1473

Genzel, R., Burkert, A., Bouché, N., et al. 2008, ApJ, 687, 59

Georgakakis, A., Hopkins, A. M., Sullivan, M., et al. 2003, MNRAS, 345, 939

Giavalisco, M., Dickinson, M., Ferguson, H. C., et al. 2004, ApJ, 600, L103

Glazebrook, K., Blake, C., Economou, F., Lilly, S., \& Colless, M. 1999,

MNRAS, 306, 843

Glazebrook, K., Abraham, R. G., McCarthy, P. J., et al. 2004, Nature, 430, 181

Greve, T. R., Ivison, R. J., Bertoldi, F., et al. 2004, MNRAS, 354, 779

Greve, T. R., Bertoldi, F., Smail, I., et al. 2005, MNRAS, 359, 1165

Gronwall, C. 1999, AIPC, 470, 335

Gronwall, C., Ciardullo, R., Hickey, Th., et al. 2007, ApJ, 667, 79

Gruppioni, C., Pozzi, F., Zamorani, G., et al. 2003, MNRAS, 341, L1

Gwyn, S. D. J., \& Hartwick, F. D. A. 2005, AJ, 130, 1337

Haarsma, D. B., Partridge, R. B., Windhorst, R. A., et al. 2000, ApJ, 544, 641

Hainline, L. J. 2008, Multi-Wavelength Properties of Submillimeter-Selected

Galaxies, Ph.D. Thesis, California Institute of Technology

Hainline, L. J., Blain, A. W., Greve, T. R., et al. 2006, ApJ, 650, 614

Hainline, L. J., Blain, A. W., Smail, I., et al. 2009, ApJ, 699, 1610

Hammer, F., Flores, H., Lilly, S. J., et al. 1997, ApJ, 481, 49

Hanish, D. J., Meurer, G. R., Ferguson, H. C., et al. 2006, ApJ, 649, 150

Helou, G., Soifer, B. T., \& Rowan-Robinson, M. 1985, ApJ, 298, L7

Hippelein, H., Maier, C., Meisenheimer, K., et al. 2003, A\&A, 402, 65

Hogg, D. W., Cohen, J. G., Blandford, R., et al. 1998, ApJ, 504, 622

Holland, W. S., Robson, E. I., Gear, W. K., et al. 1999, MNRAS, 303, 659

Hopkins, A. M. 2004, ApJ, 615, 209

Hopkins, A. M., \& Beacom, J. F. 2006, ApJ, 651, 142

Hopkins, A. M., Connolly, A. J., \& Szalay, A. S. 2000, AJ, 120, 2843

Hu, E. M., Cowie, L. L., \& McMahon, R. G. 1998, ApJ, 502, L99

Hughes, D. H., Serjeant, S., Dunlop, J., et al. 1998, Nature, 394, 241

Huynh, M. T., Pope, A., Frayer, D. T., et al. 2007, ApJ, 659, 305

Ibar, E., Cirasuolo, M., Ivison, R., et al. 2008, MNRAS, 386, 953

Iglesias-Páramo, J., Buat, V., Hernández-Fern Ãąndez, J., et al. 2007, ApJ, 670, 279

Ilbert, O., Salvato, M., Le Floc'h, E., et al. 2010, ApJ, 709, 644

Ivison, R. J., Dunlop, J. S., Smail, I., et al. 2000, ApJ, 542, 27

Ivison, R. J., Greve, T. R., Smail, I., et al. 2002, MNRAS, 337, 1

Ivison, R. J., Greve, T. R., Serjeant, S., et al. 2004, ApJS, 154, 124

Ivison, R. J., Smail, I., Dunlop, J. S., et al. 2005, MNRAS, 364, 1025

Ivison, R. J., Alexander, D. M., Biggs, A. D., et al. 2010, MNRAS, 402, 245

Iwata, I., Ohta, K., Tamura, N., et al. 2003, PASJ, 55, 415

Iwata, I., Ohta, K., Tamura, N., et al. 2007, MNRAS, 376, 1557

Kennicutt, R. C. 1998, ARA\&A, 36, 189

Knudsen, K. K., Kneib, J. P., \& Egami, E. 2008a, in Infrared Diagnostics of Galaxy Evolution, ed. R. R Chary, H. I. Teplitz, \& K. Sheth, ASP Conf. Ser., 381,372

Knudsen, K. K., van der Werf, P. P., \& Kneib, J. P. 2008b, MNRAS, 384, 1611

Knudsen, K. K., Kneib, J., Richard, J., Petitpas, G., \& Egami, E. 2010, ApJ, 709, 210

Kochanek, C. S., Pahre, M. A., Falco, E. E., et al. 2001, ApJ, 560, 566

Kodaira, K., Taniguchi, Y., Kashikawa, N., et al. 2003, PASJ, 55, L17

Kovács, A., Chapman, S. C., Dowell, C. D., et al. 2006, ApJ, 650, 592

Kudritzki, R.-P., Méndez, R. H., Feldmeier, J. J., et al. 2000, ApJ, 536, 19

Lacki, B. C., \& Thompson, T. A. 2009, ApJ, submitted [arXiv:0910.0478]

Lacki, B. C., Thompson, T. A., \& Quataert, E. 2009, ApJ, submitted, [arXiv:0907.4161]

Laurent, G. T., Glenn, J., Egami, E., et al. 2006, ApJ, 643, 38

Law, D. R., Steidel, C. C., Erb, D. K., et al. 2009, ApJ, 697, 2057

Lilly, S. J., Le Fevre, O., Hammer, F., et al. 1996, ApJ, 460, L1 
Lilly, S. J., Eales, S. A., Gear, W. K. P., et al. 1999, ApJ, 518, 641 Ly, C., Malkan, M. A., Treu, T., et al. 2009, ApJ, 697, 1410 Machalski, J., \& Godlowski, W. 2000, A\&A, 360, 463

Madau, P., Ferguson, H. C., Dickinson, M. E., et al. 1996, MNRAS, 283, 1388

Madau, P., Pozzetti, L., \& Dickinson, M. 1998, ApJ, 498, 106

Malhotra, S., \& Rhoads, J. E. 2004, ApJ, 617, L5

Mann, R. G., Oliver, S., Carballo, R., et al. 2002, MNRAS, 332, 549

Marchesini, D., van Dokkum, P. G., Förster Schreiber, N. M., et al. 2009, ApJ, 701,1765

Marleau, F. R., Fadda, D., Appleton, P. N., et al. 2007, ApJ, 663, 218

Massarotti, M., Iovino, A., \& Buzzoni, A. 2001, ApJ, 559, L105

Mauch, T., \& Sadler, E. M. 2007, MNRAS, 375, 931

Menéndez-Delmestre, K., Blain, A. W., Alexander, D. M., et al. 2007, ApJ, 655, L65

Menéndez-Delmestre, K., Blain, A. W., Smail, I., et al. 2009, ApJ, 699, 667

Mentuch, E., Abraham, R. G., Glazebrook, K., et al. 2009, ApJ, 706, 1020

Michałowski, M. J., \& Hjorth, J. 2007, in The Multicolored Landscape of Compact Objects and Their Explosive Origins, ed. L. A. Antonelli, et al. (Melville, NY: AIP), AIP Conf. Ser., 924, 143

Michałowski, M. J., Hjorth, J., Castro Cerón, J. M., et al. 2008, ApJ, 672, 817

Michałowski, M. J., Hjorth, J., Malesani, D., et al. 2009, ApJ, 693, 347

Michałowski, M. J., Watson, D., \& Hjorth, J. 2010, ApJ, 712, 942

Miller, N. A., \& Owen, F. N. 2001, AJ, 121, 1903

Mobasher, B., Dahlen, T., Hopkins, A., et al. 2009, ApJ, 690, 1074

Moorwood, A. F. M., van der Werf, P. P., Cuby, J. G., et al. 2000, A\&A, 362, 9

Murayama, T., Taniguchi, Y., Scoville, N. Z., et al. 2007, ApJS, 172, 523

Murphy, E. J. 2009, ApJ, 706, 482

Murphy, E. J., Chary, R. R., Alexander, D. M., et al. 2009, ApJ, 698, 1380

Narayanan, D., Cox, T. J., Hayward, C. C., et al. 2009, MNRAS, 400, 1919

Narayanan, D., Hayward, C. C., Cox, T. J., et al. 2010, MNRAS, 401, 1613

Nilsson, K. K., Møller, P., Möller, O., et al. 2007, A\&A, 471, 71

Nilsson, K. K., Tapken, C., Møller, P., et al. 2009, A\&A, 498, 13

Ouchi, M., Shimasaku, K., Furusawa, H., et al. 2003, ApJ, 582, 60

Ouchi, M., Shimasaku, K., Okamura, S., et al. 2004, ApJ, 611, 660

Ouchi, M., Shimasaku, K., Akiyama, M., et al. 2008, ApJS, 176, 301

Paltani, S., Le Fèvre, O., Ilbert, O., et al. 2007, A\&A, 463, 873

Palunas, P., Teplitz, H. I., Francis, P. J., Williger, G. M., \& Woodgate, B. E. 2004, ApJ, 602, 545

Pascale, E., Ade, P. A. R., Bock, J. J., et al. 2009, ApJ, 707, 1740

Pascarelle, S. M., Lanzetta, K. M., \& Fernández-Soto, A. 1998, ApJ, 508, L1

Perera, T. A., Chapin, E. L., Austermann, J. E., et al. 2008, MNRAS, 391, 1227

Pérez-González, P. G., Zamorano, J., Gallego, J., Aragón-Salamanca, A., \& Gil de Paz, A. 2003, ApJ, 591, 827

Pérez-González, P. G., Rieke, G. H., Egami, E., et al. 2005, ApJ, 630, 82 Pérez-González, P. G., Rieke, G. H., Villar, V., et al. 2008, ApJ, 675, 234 Pettini, M., Kellogg, M., Steidel, C. C., et al. 1998, ApJ, 508, 539

Pope, A., Scott, D., Dickinson, M., et al. 2006, MNRAS, 370, 1185

Pope, A., Chary, R.-R., Alexander, D. M., et al. 2008, ApJ, 675, 1171

Pozzetti, L., Bolzonella, M., Lamareille, F., et al. 2007, A\&A, 474, 443

Pozzi, F., Gruppioni, C., Oliver, S., et al. 2004, ApJ, 609, 122

Rawat, A., Hammer, F., Kembhavi, A. K., et al. 2008, ApJ, 681, 1089

Reddy, N. A., Steidel, C. C., Fadda, D., et al. 2006, ApJ, 644, 792

Reddy, N. A., Steidel, C. C., Pettini, M., et al. 2008, ApJS, 175, 48

Rhoads, J. E., Dey, A., Malhotra, S., et al. 2003, AJ, 125, 1006

Rieke, G. H., Alonso-Herrero, A., Weiner, B. J., et al. 2009, ApJ, 692, 556

Rudnick, G., Rix, H.-W., Franx, M., et al. 2003, ApJ, 599, 847

Rudnick, G., Labbé, I., Förster, S., et al. 2006, ApJ, 650, 624

Sadler, E. M., Jackson, C. A., Cannon, R. D., et al. 2002, MNRAS, 329, 227

Sajina, A., Yan, L., Lutz, D., et al. 2008, ApJ, 683, 659

Salpeter, E. E. 1955, ApJ, 121, 161

Salucci, P., \& Persic, M. 1999, MNRAS, 309, 923

Sargent, M. T., Schinnerer, E., Murphy, E., et al. 2010, ApJS, 186, 341

Santini, P., Fontana, A., Grazian, A., et al. 2009, A\&A, 504, 751

Sawicki, M., \& Thompson, D. 2006a, ApJ, 642, 653

Sawicki, M., \& Thompson, D. 2006b, ApJ, 648, 299

Sawicki, M. J., Lin, H., \& Yee, H. K. C. 1997, AJ, 113, 1
Schinnerer, E., Carilli, C. L., Capak, P., et al. 2008, ApJ, 689, L5

Scott, K. S., Austermann, J. E., Perera, T. A., et al. 2008, MNRAS, 385, 2225

Scott, S. E., Fox, M. J., Dunlop, J. S., et al. 2002, MNRAS, 331, 817

Serjeant, S., Gruppioni, C., \& Oliver, S. 2002, MNRAS, 330, 621

Serjeant, S., Dye, S., Mortier, A., et al. 2008, MNRAS, 386, 1907

Seymour, N., Dwelly, T., Moss, D., et al. 2008, MNRAS, 386, 1695

Seymour, N., Huynh, M., Dwelly, T., et al. 2009, MNRAS, 398, 1573

Shim, H., Im, M., Choi, P., Yan L., \& Storrie-Lombardi, L. 2007, ApJ, 669, 749

Shimasaku, K., Ouchi, M., Furusawa, H., et al. 2005, PASJ, 57, 447

Shimasaku, K., Kashikawa, N., Doi, M., et al. 2006, PASJ, 58, 313

Shioya, Y., Taniguchi, Y., Sasaki, S. S., et al. 2008, ApJS, 175, 128

Silva, L., Granato, G. L., Bressan, A., et al. 1998, ApJ, 509, 103

Smail, I., Ivison, R. J., Blain, A. W., et al. 2002, MNRAS, 331, 495

Smail, I., Chapman, S. C., Blain, A. W., et al. 2004, ApJ, 616, 71

Sobral, D., Best, P. N., Geach, J. E., et al. 2009, MNRAS, 398, 75

Somerville, R. S., Primack, J. R., \& Faber, S. M. 2001, MNRAS, 320, 504

Stanway, E. R., Bunker, A. J., \& McMahon, R. G. 2003, MNRAS, 342, 439

Stark, D. P., Bunker, A. J., Ellis, R. S., Eyles, L. P., \& Lacy, M. 2007, ApJ, 659, 84

Stark, D. P., Ellis, R. S., Bunker, A., et al. 2009, ApJ, 697, 1493

Steidel, C. C., Adelberger, K. L., Giavalisco, M., Dickinson M., \& Pettini, M. 1999, ApJ, 519, 1

Sullivan, M., Treyer, M. A., Ellis, R. S., et al. 2000, MNRAS, 312, 442

Swinbank, A. M., Smail, I., Chapman, S. C., et al. 2004, ApJ, 617, 64

Swinbank, A. M., Chapman, S. C., Smail, I., et al. 2006, MNRAS, 371, 465

Swinbank, A. M., Lacey, C. G., Smail, I., et al. 2008, MNRAS, 391, 420

Tacconi, L. J., Neri, R., Chapman, S. C., et al. 2006, ApJ, 640, 228

Tacconi, L. J., Genzel, R., Smail, I., et al. 2008, ApJ, 680, 246

Takagi, T., Hanami, H., \& Arimoto, N. 2004, MNRAS, 355, 424

Takata, T., Sekiguchi, K., Smail, I., et al. 2006, ApJ, 651, 713

Tamura, Y., Kohno, K., Nakanishi, K., et al. 2009, Nature, 459, 61

Taniguchi, Y., Ajiki, M., Nagao, T., et al. 2005, PASJ, 57, 165

Teplitz, H. I., Collins, N. R., Gardner, J. P., Hill, R. S., \& Rhodes, J. 2003, ApJ, 589, 704

Thompson, R. I., Eisenstein, D., Fan, X., et al. 2006, ApJ, 647, 787

Trentham, N., Blain, A. W., \& Goldader, J. 1999, MNRAS, 305, 61

Tresse, L., \& Maddox, S. J. 1998, ApJ, 495, 691

Tresse, L., Maddox, S. J., Le Fèvre, O., et al. 2002, MNRAS, 337, 369

Treyer, M. A., Ellis, R. S., Milliard, B., Donas, J., \& Bridges, T. J. 1998,

MNRAS, 300, 303

Valiante, E., Lutz, D., Sturm, E., et al. 2007, ApJ, 660, 1060

van Breukelen, C., Jarvis, M. J., \& Venemans, B. P. 2005, MNRAS, 359, 895

van de Ven, G., van Dokkum, P. G., \& Franx, M. 2003, MNRAS, 344, 924

van Dokkum, P. G., \& Franx, M. 2001, ApJ, 553, 90

Villar, V., Gallego, J., Pérez-González, P. G., et al. 2008, ApJ, 677, 169

Vlahakis, C., Eales, S., \& Dunne, L. 2007, MNRAS, 379, 1042

Wadadekar, Y., Casertano, S., \& de Mello, D. 2006, AJ, 132, 1023

Wall, J. V., Pope, A., \& Scott, D. 2008, MNRAS, 383, 435

Wang, W., Cowie, L. L., \& Barger, A. J. 2006, ApJ, 647, 74

Wang, W. H., Cowie, L. L., \& Barger, A. J. 2004, ApJ, 613, 655

Wang, W. H., Barger, A. J., \& Cowie, L. L. 2009, ApJ, 690, 319

Watabe, Y., Risaliti, G., Salvati, M., et al. 2009, MNRAS, 396, L1

Webb, T. M., Eales, S. A., Lilly, S. J., et al. 2003a, ApJ, 587, 41

Webb, T. M. A., Lilly, S. J., Clements, D. L., et al. 2003b, ApJ, 597, 680

Weiß, A., Ivison, R. J., Downes, D., et al. 2009a, ApJ, 705, L45

Weiß A., Kovács, A., Coppin, K., et al. 2009b, ApJ, 707, 1201

Wilson, G., Cowie, L. L., Barger, A. J., et al. 2002, AJ, 124, 1258

Wyder, T. K., Treyer, M. A., Milliard, B., et al. 2005, ApJ, 619, L15

Yan, L., McCarthy, P. J., Freudling, W., et al. 1999, ApJ, 519, L47

Yan, H., Dickinson, M., Giavalisco, M., et al. 2006, ApJ, 651, 24

Yang, M., Greve, T. R., Dowell, C. D., et al. 2007, ApJ, 660, 1198

Yoshida, M., Shimasaku, K., Kashikawa, N., et al. 2006, ApJ, 653, 988

Younger, J. D., Fazio, G. G., Huang, J.-S., et al. 2007, ApJ, 671, 1531

Younger, J. D., Fazio, G. G., Wilner, D. J., et al. 2008, ApJ, 688, 59

Younger, J. D., Fazio, G. G., \& Huang, J.-S. 2009a, ApJ, 704, 803

Younger, J. D., Omont, A., Fiolet, N., et al. 2009b, MNRAS, 394, 1685

Yun, M. S., Reddy, N. A., \& Condon, J. J. 2001, ApJ, 554, 803

Zheng, X. Z., Bell, E. F., Papovich, C., et al. 2007, ApJ, 661, L41

Pages 13 to 51 are available in the electronic edition of the journal at http: //wWw . aanda. org 
Appendix A: Long tables and figures.

Table A.1. Photometry detections of SMGs.

\begin{tabular}{|c|c|c|c|c|c|}
\hline SMG & $z$ & $\begin{array}{r}\lambda_{\mathrm{obs}} \\
(\mu \mathrm{m})\end{array}$ & $\begin{array}{c}\text { Flux } \\
(\mu \mathrm{Jy})\end{array}$ & $\begin{array}{l}\text { Error } \\
(\mu \mathrm{Jy})\end{array}$ & Reference \\
\hline SMMJ030226.17+000624.5 & 0.080 & 0.365 & 33.419 & 0.306 & Clements et al. (2004) \\
\hline SMMJ030226.17+000624.5 & 0.080 & 0.428 & 83.176 & 7.319 & Chapman et al. (2005) \\
\hline SMMJ030226.17+000624.5 & 0.080 & 0.440 & 83.946 & 0.232 & Clements et al. (2004) \\
\hline SMMJ030226.17+000624.5 & 0.080 & 0.550 & 181.970 & 0.335 & Clements et al. (2004) \\
\hline SMMJ030226.17+000624.5 & 0.080 & 0.656 & 275.423 & 24.234 & Chapman et al. (2005) \\
\hline SMMJ030226.17+000624.5 & 0.080 & 0.767 & 424.619 & 0.391 & Clements et al. (2004) \\
\hline SMMJ030226.17+000624.5 & 0.080 & 2.170 & 937.562 & 2.587 & Clements et al. (2004) \\
\hline SMMJ030226.17+000624.5 & 0.080 & 3.600 & 746.900 & 75.000 & Hainline (2008) \\
\hline SMMJ030226.17+000624.5 & 0.080 & 4.500 & 502.100 & 50.300 & Hainline (2008) \\
\hline SMMJ030226.17+000624.5 & 0.080 & 5.800 & 452.300 & 45.900 & Hainline (2008) \\
\hline SMMJ030226.17+000624.5 & 0.080 & 8.000 & 2220.600 & 224.100 & Hainline (2008) \\
\hline SMMJ030226.17+000624.5 & 0.080 & 24.000 & 2224.500 & 114.200 & Hainline (2008) \\
\hline SMMJ030226.17+000624.5 & 0.080 & 70.000 & 30100.000 & 7500.000 & Hainline (2008) \\
\hline SMMJ030226.17+000624.5 & 0.080 & 850.000 & 7900.000 & 1600.000 & Chapman et al. (2005) \\
\hline SMMJ030226.17+000624.5 & 0.080 & 214000.000 & 481.500 & 9.000 & Chapman et al. (2005) \\
\hline SMMJ030227.73+000653.5 & 1.408 & 0.365 & 0.637 & 0.040 & Clements et al. (2004); Menéndez-Delmestre et al. (2009) \\
\hline SMMJ030227.73+000653.5 & 1.408 & 0.428 & 1.096 & 0.096 & Chapman et al. (2005) \\
\hline SMMJ030227.73+000653.5 & 1.408 & 0.440 & 1.148 & 0.021 & Clements et al. (2004) \\
\hline SMMJ030227.73+000653.5 & 1.408 & 0.550 & 1.722 & 0.047 & Clements et al. (2004) \\
\hline SMMJ030227.73+000653.5 & 1.408 & 0.656 & 2.512 & 0.221 & Chapman et al. (2005) \\
\hline SMMJ030227.73+000653.5 & 1.408 & 0.767 & 7.416 & 0.334 & Smail et al. (2004) \\
\hline SMMJ030227.73+000653.5 & 1.408 & 0.767 & 6.310 & 0.058 & Clements et al. (2004) \\
\hline SMMJ030227.73+000653.5 & 1.408 & 1.250 & 21.782 & 0.788 & Smail et al. (2004) \\
\hline SMMJ030227.73+000653.5 & 1.408 & 2.170 & 14.494 & 0.395 & Smail et al. (2004) \\
\hline SMMJ030227.73+000653.5 & 1.408 & 2.170 & 29.107 & 0.531 & Clements et al. (2004) \\
\hline SMMJ030227.73+000653.5 & 1.408 & 3.600 & 73.800 & 7.400 & Hainline (2008) \\
\hline SMMJ030227.73+000653.5 & 1.408 & 4.500 & 79.300 & 7.900 & Hainline (2008) \\
\hline SMMJ030227.73+000653.5 & 1.408 & 5.800 & 59.800 & 6.300 & Hainline (2008) \\
\hline SMMJ030227.73+000653.5 & 1.408 & 8.000 & 61.600 & 6.600 & Hainline (2008) \\
\hline SMMJ030227.73+000653.5 & 1.408 & 24.000 & 498.900 & 33.100 & Hainline (2008) \\
\hline SMMJ030227.73+000653.5 & 1.408 & 350.000 & 42200.000 & 9800.001 & Kovács et al. (2006) \\
\hline SMMJ030227.73+000653.5 & 1.408 & 850.000 & 4400.000 & 1300.000 & Chapman et al. (2005) \\
\hline SMMJ030227.73+000653.5 & 1.408 & 214000.000 & 217.000 & 9.000 & Chapman et al. (2005) \\
\hline SMMJ030231.81+001031.3 & 1.316 & 0.550 & 0.093 & 0.029 & Clements et al. (2004); Menéndez-Delmestre et al. (2009) \\
\hline SMMJ030231.81+001031.3 & 1.316 & 4.500 & 1.600 & 0.300 & Hainline (2008) \\
\hline SMMJ030231.81+001031.3 & 1.316 & 850.000 & 5000.000 & 1500.000 & Chapman et al. (2005) \\
\hline SMMJ030231.81+001031.3 & 1.316 & 214000.000 & 45.100 & 9.000 & Chapman et al. (2005) \\
\hline SMMJ030236.15+000817.1 & 2.435 & 0.550 & 0.093 & 0.029 & Clements et al. (2004) \\
\hline SMMJ030236.15+000817.1 & 2.435 & 0.656 & 0.132 & 0.012 & Chapman et al. (2005) \\
\hline SMMJ030236.15+000817.1 & 2.435 & 2.170 & 29.107 & 0.531 & Clements et al. (2004) \\
\hline SMMJ030236.15+000817.1 & 2.435 & 3.600 & 7.700 & 0.800 & Hainline (2008) \\
\hline SMMJ030236.15+000817.1 & 2.435 & 4.500 & 9.700 & 1.000 & Hainline (2008) \\
\hline SMMJ030236.15+000817.1 & 2.435 & 5.800 & 14.900 & 2.000 & Hainline (2008) \\
\hline SMMJ030236.15+000817.1 & 2.435 & 850.000 & 3400.000 & 600.000 & Chapman et al. (2005) \\
\hline SMMJ030236.15+000817.1 & 2.435 & 214000.000 & 42.100 & 9.100 & Chapman et al. (2005) \\
\hline SMMJ030238.62+001106.3 & 0.276 & 0.365 & 0.278 & 0.034 & Clements et al. (2004) \\
\hline SMMJ030238.62+001106.3 & 0.276 & 0.428 & 0.209 & 0.018 & Chapman et al. (2005) \\
\hline SMMJ030238.62+001106.3 & 0.276 & 0.440 & 0.205 & 0.036 & Clements et al. (2004) \\
\hline SMMJ030238.62+001106.3 & 0.276 & 0.550 & 0.470 & 0.033 & Clements et al. (2004) \\
\hline SMMJ030238.62+001106.3 & 0.276 & 0.656 & 0.759 & 0.067 & Chapman et al. (2005) \\
\hline SMMJ030238.62+001106.3 & 0.276 & 0.767 & 1.350 & 0.196 & Smail et al. (2004) \\
\hline SMMJ030238.62+001106.3 & 0.276 & 0.767 & 1.076 & 0.029 & Clements et al. (2004) \\
\hline SMMJ030238.62+001106.3 & 0.276 & 2.170 & 4.066 & 0.589 & Smail et al. (2004) \\
\hline SMMJ030238.62+001106.3 & 0.276 & 2.170 & 3.733 & 0.541 & Clements et al. (2004) \\
\hline SMMJ030238.62+001106.3 & 0.276 & 3.600 & 16.600 & 1.700 & Hainline (2008) \\
\hline SMMJ030238.62+001106.3 & 0.276 & 4.500 & 19.800 & 2.000 & Hainline (2008) \\
\hline SMMJ030238.62+001106.3 & 0.276 & 850.000 & 4100.000 & 1400.000 & Chapman et al. (2005) \\
\hline SMMJ030238.62+001106.3 & 0.276 & 214000.000 & 347.300 & 9.000 & Chapman et al. (2005) \\
\hline SMMJ030244.82+000632.3 & 0.176 & 0.428 & 22.909 & 2.016 & Chapman et al. (2005) \\
\hline SMMJ030244.82+000632.3 & 0.176 & 0.656 & 83.176 & 7.319 & Chapman et al. (2005) \\
\hline SMMJ030244.82+000632.3 & 0.176 & 1.250 & 147.941 & 2.700 & Smail et al. (2004) \\
\hline SMMJ030244.82+000632.3 & 0.176 & 2.170 & 227.612 & 2.087 & Smail et al. (2004) \\
\hline SMMJ030244.82+000632.3 & 0.176 & 850.000 & 4900.000 & 1100.000 & Chapman et al. (2005) \\
\hline
\end{tabular}


Table A.1. continued

\begin{tabular}{|c|c|c|c|c|c|}
\hline SMG & $z$ & $\begin{array}{r}\lambda_{\text {obs }} \\
(\mu \mathrm{m})\end{array}$ & $\begin{array}{c}\text { Flux } \\
(\mu \mathrm{Jy})\end{array}$ & $\begin{array}{l}\text { Error } \\
(\mu \mathrm{Jy})\end{array}$ & Reference \\
\hline SMMJ105151.69+572636.0 & 1.147 & 0.656 & 0.302 & 0.027 & Chapman et al. (2005); Menéndez-Delmestre et al. (2009) \\
\hline SMMJ105151.69+572636.0 & 1.147 & 0.656 & 0.340 & 0.021 & Ivison et al. (2005) \\
\hline SMMJ105151.69+572636.0 & 1.147 & 0.767 & 2.302 & 0.203 & Ivison et al. (2002) \\
\hline SMMJ105151.69+572636.0 & 1.147 & 2.170 & 44.177 & 2.375 & Ivison et al. (2002) \\
\hline SMMJ105151.69+572636.0 & 1.147 & 3.600 & 78.100 & 7.800 & Hainline (2008) \\
\hline SMMJ105151.69+572636.0 & 1.147 & 4.500 & 89.200 & 8.900 & Hainline (2008) \\
\hline SMMJ105151.69+572636.0 & 1.147 & 5.800 & 64.700 & 6.900 & Hainline (2008) \\
\hline SMMJ105151.69+572636.0 & 1.147 & 8.000 & 63.300 & 6.600 & Hainline (2008) \\
\hline SMMJ105151.69+572636.0 & 1.147 & 24.000 & 342.400 & 24.000 & Hainline (2008) \\
\hline SMMJ105151.69+572636.0 & 1.147 & 850.000 & 6700.000 & 1700.000 & Chapman et al. (2005) \\
\hline SMMJ105151.69+572636.0 & 1.147 & 1200.000 & 1600.000 & 600.000 & Ivison et al. (2005) \\
\hline SMMJ105151.69+572636.0 & 1.147 & 214000.000 & 134.400 & 13.000 & Chapman et al. (2005) \\
\hline SMMJ105155.47+572312.7 & 2.686 & 0.428 & 1.096 & 0.096 & Chapman et al. (2005); Valiante et al. (2007) \\
\hline SMMJ105155.47+572312.7 & 2.686 & 0.656 & 1.905 & 0.168 & Chapman et al. (2005) \\
\hline SMMJ105155.47+572312.7 & 2.686 & 0.656 & 0.603 & 0.016 & Ivison et al. (2005) \\
\hline SMMJ105155.47+572312.7 & 2.686 & 3.600 & 5.100 & 0.500 & Hainline (2008) \\
\hline SMMJ105155.47+572312.7 & 2.686 & 4.500 & 7.600 & 0.800 & Hainline (2008) \\
\hline SMMJ105155.47+572312.7 & 2.686 & 5.800 & 13.100 & 2.100 & Hainline (2008) \\
\hline SMMJ105155.47+572312.7 & 2.686 & 8.000 & 18.500 & 2.000 & Hainline (2008) \\
\hline SMMJ105155.47+572312.7 & 2.686 & 24.000 & 99.000 & 15.900 & Hainline (2008) \\
\hline SMMJ105155.47+572312.7 & 2.686 & 850.000 & 5700.000 & 1400.000 & Chapman et al. (2005) \\
\hline SMMJ105155.47+572312.7 & 2.686 & 1200.000 & 3300.000 & 800.000 & Greve et al. (2004) \\
\hline SMMJ105155.47+572312.7 & 2.686 & 61182.000 & 38.000 & 19.000 & Ivison et al. (2002) \\
\hline SMMJ105155.47+572312.7 & 2.686 & 214000.000 & 46.300 & 10.200 & Chapman et al. (2005) \\
\hline SMMJ105158.02+571800.2 & 2.239 & 0.656 & 0.832 & 0.073 & Chapman et al. (2005); Menéndez-Delmestre et al. (2009) \\
\hline SMMJ105158.02+571800.2 & 2.239 & 0.656 & 1.047 & 0.029 & Ivison et al. (2005) \\
\hline SMMJ105158.02+571800.2 & 2.239 & 0.767 & 1.231 & 0.159 & Ivison et al. (2002) \\
\hline SMMJ105158.02+571800.2 & 2.239 & 2.170 & 18.758 & 1.492 & Ivison et al. (2002) \\
\hline SMMJ105158.02+571800.2 & 2.239 & 3.600 & 53.300 & 5.300 & Hainline (2008) \\
\hline SMMJ105158.02+571800.2 & 2.239 & 4.500 & 55.500 & 5.600 & Hainline (2008) \\
\hline SMMJ105158.02+571800.2 & 2.239 & 5.800 & 43.900 & 5.100 & Hainline (2008) \\
\hline SMMJ105158.02+571800.2 & 2.239 & 8.000 & 53.000 & 5.800 & Hainline (2008) \\
\hline SMMJ105158.02+571800.2 & 2.239 & 24.000 & 241.100 & 21.100 & Hainline (2008) \\
\hline SMMJ105158.02+571800.2 & 2.239 & 850.000 & 7700.000 & 1700.000 & Chapman et al. (2005) \\
\hline SMMJ105158.02+571800.2 & 2.239 & 1200.000 & 2900.000 & 700.000 & Greve et al. (2004) \\
\hline SMMJ105158.02+571800.2 & 2.239 & 61182.000 & 109.000 & 26.000 & Ivison et al. (2002) \\
\hline SMMJ105158.02+571800.2 & 2.239 & 214000.000 & 98.100 & 11.600 & Chapman et al. (2005) \\
\hline SMMJ105200.22+572420.2 & 0.689 & 0.656 & 5.248 & 0.462 & Chapman et al. (2005); Menéndez-Delmestre et al. (2009) \\
\hline SMMJ105200.22+572420.2 & 0.689 & 0.656 & 1.871 & 0.034 & Ivison et al. (2005) \\
\hline SMMJ105200.22+572420.2 & 0.689 & 0.767 & 5.626 & 0.153 & Ivison et al. (2002) \\
\hline SMMJ105200.22+572420.2 & 0.689 & 2.170 & 19.462 & 1.548 & Ivison et al. (2002) \\
\hline SMMJ105200.22+572420.2 & 0.689 & 3.600 & 22.500 & 2.300 & Hainline (2008) \\
\hline SMMJ105200.22+572420.2 & 0.689 & 4.500 & 25.900 & 2.600 & Hainline (2008) \\
\hline SMMJ105200.22+572420.2 & 0.689 & 5.800 & 40.800 & 4.400 & Hainline (2008) \\
\hline SMMJ105200.22+572420.2 & 0.689 & 8.000 & 96.500 & 9.700 & Hainline (2008) \\
\hline SMMJ105200.22+572420.2 & 0.689 & 24.000 & 282.000 & 59.000 & Egami et al. (2004) \\
\hline SMMJ105200.22+572420.2 & 0.689 & 70.000 & 6200.000 & 1300.000 & Hainline (2008) \\
\hline SMMJ105200.22+572420.2 & 0.689 & 350.000 & 15500.000 & 5500.000 & Laurent et al. (2006) \\
\hline SMMJ105200.22+572420.2 & 0.689 & 850.000 & 5100.000 & 1300.000 & Chapman et al. (2005) \\
\hline SMMJ105200.22+572420.2 & 0.689 & 1100.000 & 4000.000 & 1300.000 & Laurent et al. (2006) \\
\hline SMMJ105200.22+572420.2 & 0.689 & 1200.000 & 2400.000 & 600.000 & Greve et al. (2004) \\
\hline SMMJ105200.22+572420.2 & 0.689 & 61182.000 & 57.000 & 32.000 & Ivison et al. (2002) \\
\hline SMMJ105200.22+572420.2 & 0.689 & 214000.000 & 57.400 & 13.200 & Chapman et al. (2005) \\
\hline SMMJ105201.25+572445.7 & 2.148 & 0.656 & 0.191 & 0.017 & Chapman et al. (2005) \\
\hline SMMJ105201.25+572445.7 & 2.148 & 0.656 & 0.150 & 0.018 & Ivison et al. (2005) \\
\hline SMMJ105201.25+572445.7 & 2.148 & 2.170 & 7.892 & 1.328 & Ivison et al. (2002) \\
\hline SMMJ105201.25+572445.7 & 2.148 & 3.600 & 5.500 & 0.600 & Hainline (2008) \\
\hline SMMJ105201.25+572445.7 & 2.148 & 4.500 & 8.700 & 0.900 & Hainline (2008) \\
\hline SMMJ105201.25+572445.7 & 2.148 & 5.800 & 11.900 & 2.200 & Hainline (2008) \\
\hline SMMJ105201.25+572445.7 & 2.148 & 8.000 & 14.800 & 1.900 & Hainline (2008) \\
\hline SMMJ105201.25+572445.7 & 2.148 & 24.000 & 172.500 & 16.300 & Hainline (2008) \\
\hline SMMJ105201.25+572445.7 & 2.148 & 350.000 & 24100.000 & 5500.000 & Laurent et al. (2006) \\
\hline SMMJ105201.25+572445.7 & 2.148 & 850.000 & 9900.000 & 2200.000 & Chapman et al. (2005) \\
\hline
\end{tabular}


Table A.1. continued

\begin{tabular}{|c|c|c|c|c|c|}
\hline SMG & $z$ & $\begin{array}{r}\lambda_{\mathrm{obs}} \\
(\mu \mathrm{m})\end{array}$ & $\begin{array}{r}\text { Flux } \\
(\mu \mathrm{Jy})\end{array}$ & $\begin{array}{l}\text { Error } \\
(\mu \mathrm{Jy})\end{array}$ & Reference \\
\hline SMMJ105201.25+572445.7 & 2.148 & 1100.000 & 4400.000 & 1300.000 & Laurent et al. (2006) \\
\hline SMMJ105201.25+572445.7 & 2.148 & 1200.000 & 3400.000 & 600.000 & Greve et al. (2004) \\
\hline SMMJ105201.25+572445.7 & 2.148 & 61182.000 & 56.000 & 37.000 & Ivison et al. (2002) \\
\hline SMMJ105201.25+572445.7 & 2.148 & 214000.000 & 72.100 & 10.200 & Chapman et al. (2005) \\
\hline SMMJ105207.49+571904.0 & 2.689 & 0.656 & 0.145 & 0.013 & Chapman et al. (2005); Valiante et al. (2007) \\
\hline SMMJ105207.49+571904.0 & 2.689 & 0.767 & 2.010 & 0.149 & Ivison et al. (2002) \\
\hline SMMJ105207.49+571904.0 & 2.689 & 0.767 & 0.352 & 0.108 & Smail et al. (2004) \\
\hline SMMJ105207.49+571904.0 & 2.689 & 3.600 & 10.100 & 1.000 & Hainline (2008) \\
\hline SMMJ105207.49+571904.0 & 2.689 & 4.500 & 12.900 & 1.300 & Hainline (2008) \\
\hline SMMJ105207.49+571904.0 & 2.689 & 24.000 & 190.900 & 14.800 & Hainline (2008) \\
\hline SMMJ105207.49+571904.0 & 2.689 & 350.000 & 38000.000 & 7200.000 & Kovács et al. (2006) \\
\hline SMMJ105207.49+571904.0 & 2.689 & 850.000 & 6200.000 & 1600.000 & Chapman et al. (2005) \\
\hline SMMJ105207.49+571904.0 & 2.689 & 61182.000 & 380.000 & 28.000 & Ivison et al. (2002) \\
\hline SMMJ105207.49+571904.0 & 2.689 & 214000.000 & 277.900 & 11.900 & Chapman et al. (2005) \\
\hline SMMJ105225.79+571906.4 & 2.372 & 0.656 & 0.479 & 0.042 & Chapman et al. (2005) \\
\hline SMMJ105225.79+571906.4 & 2.372 & 0.767 & 4.766 & 0.130 & Smail et al. (2004) \\
\hline SMMJ105225.79+571906.4 & 2.372 & 2.170 & 19.462 & 1.875 & Smail et al. (2004) \\
\hline SMMJ105225.79+571906.4 & 2.372 & 3.600 & 28.400 & 2.900 & Hainline (2008) \\
\hline SMMJ105225.79+571906.4 & 2.372 & 4.500 & 22.300 & 2.300 & Hainline (2008) \\
\hline SMMJ105225.79+571906.4 & 2.372 & 5.800 & 25.900 & 3.700 & Hainline (2008) \\
\hline SMMJ105225.79+571906.4 & 2.372 & 8.000 & 37.300 & 5.000 & Hainline (2008) \\
\hline SMMJ105225.79+571906.4 & 2.372 & 24.000 & 178.600 & 14.400 & Hainline (2008) \\
\hline SMMJ105225.79+571906.4 & 2.372 & 850.000 & 4900.000 & 1500.000 & Chapman et al. (2005) \\
\hline SMMJ105225.79+571906.4 & 2.372 & 214000.000 & 127.400 & 5.100 & Chapman et al. (2005) \\
\hline SMMJ105227.58+572512.4 & 2.142 & 0.656 & 0.363 & 0.032 & Chapman et al. (2005); Menéndez-Delmestre et al. (2009) \\
\hline SMMJ105227.58+572512.4 & 2.142 & 0.656 & 0.310 & 0.017 & Ivison et al. (2005) \\
\hline SMMJ105227.58+572512.4 & 2.142 & 0.767 & 0.646 & 0.142 & Ivison et al. (2002) \\
\hline SMMJ105227.58+572512.4 & 2.142 & 2.170 & 11.945 & 0.538 & Ivison et al. (2002) \\
\hline SMMJ105227.58+572512.4 & 2.142 & 3.600 & 19.600 & 2.000 & Hainline (2008) \\
\hline SMMJ105227.58+572512.4 & 2.142 & 4.500 & 25.600 & 2.600 & Hainline (2008) \\
\hline SMMJ105227.58+572512.4 & 2.142 & 5.800 & 41.400 & 4.900 & Hainline (2008) \\
\hline SMMJ105227.58+572512.4 & 2.142 & 8.000 & 28.500 & 3.000 & Hainline (2008) \\
\hline SMMJ105227.58+572512.4 & 2.142 & 24.000 & 226.300 & 17.400 & Hainline (2008) \\
\hline SMMJ105227.58+572512.4 & 2.142 & 350.000 & 44000.000 & 16000.000 & Laurent et al. (2006) \\
\hline SMMJ105227.58+572512.4 & 2.142 & 850.000 & 4500.000 & 1300.000 & Chapman et al. (2005) \\
\hline SMMJ105227.58+572512.4 & 2.142 & 1100.000 & 4100.000 & 1300.000 & Laurent et al. (2006) \\
\hline SMMJ105227.58+572512.4 & 2.142 & 1200.000 & 2800.000 & 500.000 & Greve et al. (2004) \\
\hline SMMJ105227.58+572512.4 & 2.142 & 61182.000 & 32.000 & 22.000 & Ivison et al. (2002) \\
\hline SMMJ105227.58+572512.4 & 2.142 & 214000.000 & 39.200 & 11.400 & Chapman et al. (2005) \\
\hline SMMJ105227.77+572218.2 & 1.956 & 0.656 & 0.145 & 0.013 & Chapman et al. (2005) \\
\hline SMMJ105227.77+572218.2 & 1.956 & 850.000 & 7000.000 & 2100.000 & Chapman et al. (2005) \\
\hline SMMJ105227.77+572218.2 & 1.956 & 1100.000 & 5100.000 & 1300.000 & Laurent et al. (2006) \\
\hline SMMJ105227.77+572218.2 & 1.956 & 1200.000 & 3100.000 & 700.000 & Greve et al. (2004) \\
\hline SMMJ105227.77+572218.2 & 1.956 & 214000.000 & 40.400 & 9.400 & Chapman et al. (2005) \\
\hline SMMJ105230.73+572209.5 & 2.611 & 0.656 & 1.738 & 0.153 & Chapman et al. (2005) \\
\hline SMMJ105230.73+572209.5 & 2.611 & 0.656 & 2.399 & 0.044 & Ivison et al. (2005) \\
\hline SMMJ105230.73+572209.5 & 2.611 & 0.767 & 2.005 & 0.125 & Smail et al. (2004) \\
\hline SMMJ105230.73+572209.5 & 2.611 & 2.170 & 13.465 & 1.845 & Smail et al. (2004) \\
\hline SMMJ105230.73+572209.5 & 2.611 & 3.600 & 33.400 & 3.400 & Hainline (2008) \\
\hline SMMJ105230.73+572209.5 & 2.611 & 4.500 & 39.500 & 4.000 & Hainline (2008) \\
\hline SMMJ105230.73+572209.5 & 2.611 & 5.800 & 56.500 & 6.500 & Hainline (2008) \\
\hline SMMJ105230.73+572209.5 & 2.611 & 8.000 & 46.600 & 4.700 & Hainline (2008) \\
\hline SMMJ105230.73+572209.5 & 2.611 & 24.000 & 184.200 & 17.900 & Hainline (2008) \\
\hline SMMJ105230.73+572209.5 & 2.611 & 350.000 & 41000.000 & 6800.000 & Kovács et al. (2006) \\
\hline SMMJ105230.73+572209.5 & 2.611 & 850.000 & 11000.000 & 2600.000 & Chapman et al. (2005) \\
\hline SMMJ105230.73+572209.5 & 2.611 & 1100.000 & 5100.000 & 1300.000 & Laurent et al. (2006) \\
\hline SMMJ105230.73+572209.5 & 2.611 & 1200.000 & 2900.000 & 700.000 & Greve et al. (2004) \\
\hline SMMJ105230.73+572209.5 & 2.611 & 61182.000 & 60.000 & 35.000 & Ivison et al. (2002) \\
\hline SMMJ105230.73+572209.5 & 2.611 & 214000.000 & 54.000 & 14.000 & Ivison et al. (2002) \\
\hline SMMJ105230.73+572209.5 & 2.611 & 214000.000 & 86.300 & 15.400 & Chapman et al. (2005) \\
\hline SMMJ105238.19+571651.1 & 1.852 & 0.656 & 3.020 & 0.266 & Chapman et al. (2005); Menéndez-Delmestre et al. (2009) \\
\hline SMMJ105238.19+571651.1 & 1.852 & 0.767 & 2.010 & 0.113 & Smail et al. (2004) \\
\hline SMMJ105238.19+571651.1 & 1.852 & 3.600 & 17.400 & 1.800 & Hainline (2008) \\
\hline
\end{tabular}


Table A.1. continued

\begin{tabular}{|c|c|c|c|c|c|}
\hline SMG & $z$ & $\begin{array}{r}\lambda_{\mathrm{obs}} \\
(\mu \mathrm{m})\end{array}$ & $\begin{array}{c}\text { Flux } \\
(\mu \mathrm{Jy})\end{array}$ & $\begin{array}{l}\text { Error } \\
(\mu \mathrm{Jy})\end{array}$ & Reference \\
\hline SMMJ105238.19+571651.1 & 1.852 & 4.500 & 18.800 & 2.000 & Hainline (2008) \\
\hline SMMJ105238.19+571651.1 & 1.852 & 5.800 & 31.100 & 4.300 & Hainline (2008) \\
\hline SMMJ105238.19+571651.1 & 1.852 & 24.000 & 498.300 & 29.600 & Hainline (2008) \\
\hline SMMJ105238.19+571651.1 & 1.852 & 850.000 & 5300.000 & 1600.000 & Chapman et al. (2005) \\
\hline SMMJ105238.19+571651.1 & 1.852 & 214000.000 & 71.100 & 12.600 & Chapman et al. (2005) \\
\hline SMMJ105238.30+572435.8 & 3.036 & 0.656 & 0.525 & 0.046 & Chapman et al. (2005); Menéndez-Delmestre et al. (2009) \\
\hline SMMJ105238.30+572435.8 & 3.036 & 0.656 & 0.167 & 0.012 & Ivison et al. (2005) \\
\hline SMMJ105238.30+572435.8 & 3.036 & 0.767 & 1.208 & 0.166 & Ivison et al. (2002) \\
\hline SMMJ105238.30+572435.8 & 3.036 & 2.170 & 4.889 & 0.970 & Ivison et al. (2002) \\
\hline SMMJ105238.30+572435.8 & 3.036 & 3.600 & 28.900 & 2.900 & Hainline (2008) \\
\hline SMMJ105238.30+572435.8 & 3.036 & 4.500 & 32.700 & 3.300 & Hainline (2008) \\
\hline SMMJ105238.30+572435.8 & 3.036 & 5.800 & 29.800 & 4.600 & Hainline (2008) \\
\hline SMMJ105238.30+572435.8 & 3.036 & 24.000 & 335.600 & 19.400 & Hainline (2008) \\
\hline SMMJ105238.30+572435.8 & 3.036 & 350.000 & 40500.000 & 6500.000 & Kovács et al. (2006) \\
\hline SMMJ105238.30+572435.8 & 3.036 & 850.000 & 10900.000 & 2400.000 & Chapman et al. (2005) \\
\hline SMMJ105238.30+572435.8 & 3.036 & 1100.000 & 4800.000 & 1300.000 & Laurent et al. (2006) \\
\hline SMMJ105238.30+572435.8 & 3.036 & 1200.000 & 4800.000 & 600.000 & Greve et al. (2004) \\
\hline SMMJ105238.30+572435.8 & 3.036 & 214000.000 & 29.000 & 11.000 & Ivison et al. (2002) \\
\hline SMMJ105238.30+572435.8 & 3.036 & 214000.000 & 61.000 & 22.000 & Chapman et al. (2005) \\
\hline SMMJ123549.44+621536.8 & 2.203 & 0.365 & 0.518 & 0.046 & Capak et al. (2004); Menéndez-Delmestre et al. (2009) \\
\hline SMMJ123549.44+621536.8 & 2.203 & 0.428 & 0.759 & 0.067 & Chapman et al. (2005) \\
\hline SMMJ123549.44+621536.8 & 2.203 & 0.443 & 0.975 & 0.062 & Capak et al. (2004) \\
\hline SMMJ123549.44+621536.8 & 2.203 & 0.547 & 1.369 & 0.045 & Capak et al. (2004) \\
\hline SMMJ123549.44+621536.8 & 2.203 & 0.653 & 1.790 & 0.043 & Capak et al. (2004) \\
\hline SMMJ123549.44+621536.8 & 2.203 & 0.656 & 1.202 & 0.106 & Chapman et al. (2005) \\
\hline SMMJ123549.44+621536.8 & 2.203 & 0.767 & 2.324 & 0.042 & Smail et al. (2004) \\
\hline SMMJ123549.44+621536.8 & 2.203 & 0.798 & 2.273 & 0.090 & Capak et al. (2004) \\
\hline SMMJ123549.44+621536.8 & 2.203 & 0.907 & 2.656 & 0.125 & Capak et al. (2004) \\
\hline SMMJ123549.44+621536.8 & 2.203 & 1.250 & 7.483 & 0.402 & Smail et al. (2004) \\
\hline SMMJ123549.44+621536.8 & 2.203 & 1.895 & 9.213 & 1.507 & Capak et al. (2004) \\
\hline SMMJ123549.44+621536.8 & 2.203 & 24.000 & 178.700 & 31.400 & Hainline (2008) \\
\hline SMMJ123549.44+621536.8 & 2.203 & 850.000 & 8300.001 & 2500.000 & Chapman et al. (2005) \\
\hline SMMJ123549.44+621536.8 & 2.203 & 1300.000 & 2000.000 & 600.000 & Tacconi et al. (2006) \\
\hline SMMJ123549.44+621536.8 & 2.203 & 214000.000 & 74.600 & 9.500 & Chapman et al. (2005) \\
\hline SMMJ123553.26+621337.7 & 2.098 & 0.365 & 0.654 & 0.049 & Capak et al. (2004); Menéndez-Delmestre et al. (2009) \\
\hline SMMJ123553.26+621337.7 & 2.098 & 0.428 & 0.437 & 0.038 & Chapman et al. (2005) \\
\hline SMMJ123553.26+621337.7 & 2.098 & 0.443 & 0.633 & 0.046 & Capak et al. (2004) \\
\hline SMMJ123553.26+621337.7 & 2.098 & 0.547 & 0.560 & 0.033 & Capak et al. (2004) \\
\hline SMMJ123553.26+621337.7 & 2.098 & 0.573 & 0.233 & 0.021 & Chapman et al. (2003b) \\
\hline SMMJ123553.26+621337.7 & 2.098 & 0.653 & 0.659 & 0.032 & Capak et al. (2004) \\
\hline SMMJ123553.26+621337.7 & 2.098 & 0.656 & 0.479 & 0.042 & Chapman et al. (2005) \\
\hline SMMJ123553.26+621337.7 & 2.098 & 0.767 & 0.282 & 0.025 & Chapman et al. (2003b) \\
\hline SMMJ123553.26+621337.7 & 2.098 & 0.767 & 0.676 & 0.042 & Smail et al. (2004) \\
\hline SMMJ123553.26+621337.7 & 2.098 & 0.798 & 0.658 & 0.063 & Capak et al. (2004) \\
\hline SMMJ123553.26+621337.7 & 2.098 & 0.907 & 0.900 & 0.092 & Capak et al. (2004) \\
\hline SMMJ123553.26+621337.7 & 2.098 & 1.250 & 1.915 & 0.422 & Smail et al. (2004) \\
\hline SMMJ123553.26+621337.7 & 2.098 & 2.170 & 2.973 & 0.676 & Smail et al. (2004) \\
\hline SMMJ123553.26+621337.7 & 2.098 & 3.600 & 9.200 & 1.300 & Hainline (2008) \\
\hline SMMJ123553.26+621337.7 & 2.098 & 4.500 & 14.600 & 1.700 & Hainline (2008) \\
\hline SMMJ123553.26+621337.7 & 2.098 & 5.800 & 18.100 & 1.900 & Hainline (2008) \\
\hline SMMJ123553.26+621337.7 & 2.098 & 8.000 & 24.700 & 2.700 & Hainline (2008) \\
\hline SMMJ123553.26+621337.7 & 2.098 & 850.000 & 8800.001 & 2100.000 & Chapman et al. (2005) \\
\hline SMMJ123553.26+621337.7 & 2.098 & 214000.000 & 58.400 & 9.000 & Chapman et al. (2005) \\
\hline SMMJ123555.14+620901.7 & 1.875 & 0.365 & 0.640 & 0.054 & Capak et al. (2004); Pope et al. (2008) \\
\hline SMMJ123555.14+620901.7 & 1.875 & 0.428 & 0.575 & 0.051 & Chapman et al. (2005) \\
\hline SMMJ123555.14+620901.7 & 1.875 & 0.443 & 0.728 & 0.051 & Capak et al. (2004) \\
\hline SMMJ123555.14+620901.7 & 1.875 & 0.547 & 0.818 & 0.038 & Capak et al. (2004) \\
\hline SMMJ123555.14+620901.7 & 1.875 & 0.653 & 1.093 & 0.037 & Capak et al. (2004) \\
\hline SMMJ123555.14+620901.7 & 1.875 & 0.656 & 0.759 & 0.067 & Chapman et al. (2005) \\
\hline SMMJ123555.14+620901.7 & 1.875 & 0.767 & 1.915 & 0.035 & Smail et al. (2004) \\
\hline SMMJ123555.14+620901.7 & 1.875 & 0.798 & 1.520 & 0.084 & Capak et al. (2004) \\
\hline SMMJ123555.14+620901.7 & 1.875 & 0.907 & 1.983 & 0.113 & Capak et al. (2004) \\
\hline SMMJ123555.14+620901.7 & 1.875 & 1.250 & 7.622 & 0.606 & Smail et al. (2004) \\
\hline SMMJ123555.14+620901.7 & 1.875 & 2.170 & 17.426 & 1.088 & Smail et al. (2004) \\
\hline SMMJ123555.14+620901.7 & 1.875 & 3.600 & 42.500 & 4.900 & Hainline (2008) \\
\hline
\end{tabular}


Table A.1. continued

\begin{tabular}{|c|c|c|c|c|c|}
\hline SMG & $z$ & $\begin{array}{r}\lambda_{\mathrm{obs}} \\
(\mu \mathrm{m})\end{array}$ & $\begin{array}{r}\text { Flux } \\
(\mu \mathrm{Jy})\end{array}$ & $\begin{array}{l}\text { Error } \\
(\mu \mathrm{Jy})\end{array}$ & Reference \\
\hline SMMJ123555.14+620901.7 & 1.875 & 4.500 & 61.500 & 7.000 & Hainline (2008) \\
\hline SMMJ123555.14+620901.7 & 1.875 & 5.800 & 79.500 & 8.300 & Hainline (2008) \\
\hline SMMJ123555.14+620901.7 & 1.875 & 8.000 & 93.800 & 10.200 & Hainline (2008) \\
\hline SMMJ123555.14+620901.7 & 1.875 & 24.000 & 333.500 & 22.200 & Hainline (2008) \\
\hline SMMJ123555.14+620901.7 & 1.875 & 850.000 & 5400.000 & 1900.000 & Chapman et al. (2005) \\
\hline SMMJ123555.14+620901.7 & 1.875 & 214000.000 & 212.000 & 13.700 & Chapman et al. (2005) \\
\hline SMMJ123600.10+620253.5 & 2.710 & 0.365 & 0.105 & 0.025 & Capak et al. (2004) \\
\hline SMMJ123600.10+620253.5 & 2.710 & 0.428 & 0.191 & 0.017 & Chapman et al. (2005) \\
\hline SMMJ123600.10+620253.5 & 2.710 & 0.443 & 0.272 & 0.031 & Capak et al. (2004) \\
\hline SMMJ123600.10+620253.5 & 2.710 & 0.547 & 0.314 & 0.029 & Capak et al. (2004) \\
\hline SMMJ123600.10+620253.5 & 2.710 & 0.573 & 0.139 & 0.012 & Chapman et al. (2003b) \\
\hline SMMJ123600.10+620253.5 & 2.710 & 0.653 & 0.435 & 0.028 & Capak et al. (2004) \\
\hline SMMJ123600.10+620253.5 & 2.710 & 0.656 & 0.251 & 0.022 & Chapman et al. (2005) \\
\hline SMMJ123600.10+620253.5 & 2.710 & 0.767 & 0.330 & 0.029 & Chapman et al. (2003b) \\
\hline SMMJ123600.10+620253.5 & 2.710 & 0.767 & 0.415 & 0.033 & Smail et al. (2004) \\
\hline SMMJ123600.10+620253.5 & 2.710 & 0.798 & 0.354 & 0.058 & Capak et al. (2004) \\
\hline SMMJ123600.10+620253.5 & 2.710 & 0.907 & 0.435 & 0.076 & Capak et al. (2004) \\
\hline SMMJ123600.10+620253.5 & 2.710 & 850.000 & 6900.000 & 2000.000 & Chapman et al. (2005) \\
\hline SMMJ123600.10+620253.5 & 2.710 & 214000.000 & 262.000 & 17.100 & Chapman et al. (2005) \\
\hline SMMJ123600.15+621047.2 & 1.994 & 0.365 & 0.075 & 0.024 & Capak et al. (2004); Pope et al. (2008) \\
\hline SMMJ123600.15+621047.2 & 1.994 & 0.428 & 0.251 & 0.022 & Chapman et al. (2005) \\
\hline SMMJ123600.15+621047.2 & 1.994 & 0.653 & 0.061 & 0.016 & Capak et al. (2004) \\
\hline SMMJ123600.15+621047.2 & 1.994 & 0.656 & 0.331 & 0.029 & Chapman et al. (2005) \\
\hline SMMJ123600.15+621047.2 & 1.994 & 0.767 & 1.426 & 0.039 & Smail et al. (2004) \\
\hline SMMJ123600.15+621047.2 & 1.994 & 0.798 & 0.106 & 0.034 & Capak et al. (2004) \\
\hline SMMJ123600.15+621047.2 & 1.994 & 1.250 & 5.421 & 0.477 & Smail et al. (2004) \\
\hline SMMJ123600.15+621047.2 & 1.994 & 2.170 & 6.445 & 0.832 & Smail et al. (2004) \\
\hline SMMJ123600.15+621047.2 & 1.994 & 3.600 & 12.600 & 1.500 & Hainline (2008) \\
\hline SMMJ123600.15+621047.2 & 1.994 & 4.500 & 14.800 & 1.700 & Hainline (2008) \\
\hline SMMJ123600.15+621047.2 & 1.994 & 5.800 & 27.000 & 2.800 & Hainline (2008) \\
\hline SMMJ123600.15+621047.2 & 1.994 & 8.000 & 61.900 & 6.400 & Hainline (2008) \\
\hline SMMJ123600.15+621047.2 & 1.994 & 24.000 & 1270.800 & 64.000 & Hainline (2008) \\
\hline SMMJ123600.15+621047.2 & 1.994 & 350.000 & 22300.000 & 6300.000 & Kovács et al. (2006) \\
\hline SMMJ123600.15+621047.2 & 1.994 & 850.000 & 7900.000 & 2400.000 & Chapman et al. (2005) \\
\hline SMMJ123600.15+621047.2 & 1.994 & 214000.000 & 131.000 & 10.600 & Chapman et al. (2005) \\
\hline SMMJ123606.72+621550.7 & 2.416 & 0.365 & 1.144 & 0.065 & Capak et al. (2004) \\
\hline SMMJ123606.72+621550.7 & 2.416 & 0.428 & 1.445 & 0.127 & Chapman et al. (2005) \\
\hline SMMJ123606.72+621550.7 & 2.416 & 0.443 & 1.701 & 0.090 & Capak et al. (2004) \\
\hline SMMJ123606.72+621550.7 & 2.416 & 0.547 & 1.827 & 0.054 & Capak et al. (2004) \\
\hline SMMJ123606.72+621550.7 & 2.416 & 0.653 & 1.800 & 0.044 & Capak et al. (2004) \\
\hline SMMJ123606.72+621550.7 & 2.416 & 0.656 & 1.318 & 0.116 & Chapman et al. (2005) \\
\hline SMMJ123606.72+621550.7 & 2.416 & 0.775 & 1.738 & 0.153 & Pope et al. (2006) \\
\hline SMMJ123606.72+621550.7 & 2.416 & 0.798 & 2.099 & 0.095 & Capak et al. (2004) \\
\hline SMMJ123606.72+621550.7 & 2.416 & 0.907 & 2.169 & 0.124 & Capak et al. (2004) \\
\hline SMMJ123606.72+621550.7 & 2.416 & 3.600 & 9.600 & 1.400 & Hainline (2008) \\
\hline SMMJ123606.72+621550.7 & 2.416 & 4.500 & 13.200 & 1.600 & Hainline (2008) \\
\hline SMMJ123606.72+621550.7 & 2.416 & 5.800 & 19.400 & 2.100 & Hainline (2008) \\
\hline SMMJ123606.72+621550.7 & 2.416 & 8.000 & 26.300 & 2.900 & Hainline (2008) \\
\hline SMMJ123606.72+621550.7 & 2.416 & 24.000 & 124.000 & 8.600 & Hainline (2008) \\
\hline SMMJ123606.72+621550.7 & 2.416 & 850.000 & 4400.000 & 1400.000 & Chapman et al. (2005) \\
\hline SMMJ123606.72+621550.7 & 2.416 & 214000.000 & 24.000 & 5.900 & Chapman et al. (2005) \\
\hline SMMJ123606.85+621021.4 & 2.509 & 0.365 & 0.123 & 0.030 & Capak et al. (2004) \\
\hline SMMJ123606.85+621021.4 & 2.509 & 0.428 & 0.209 & 0.018 & Chapman et al. (2005) \\
\hline SMMJ123606.85+621021.4 & 2.509 & 0.443 & 0.288 & 0.036 & Capak et al. (2004) \\
\hline SMMJ123606.85+621021.4 & 2.509 & 0.547 & 0.328 & 0.031 & Capak et al. (2004) \\
\hline SMMJ123606.85+621021.4 & 2.509 & 0.653 & 0.623 & 0.032 & Capak et al. (2004) \\
\hline SMMJ123606.85+621021.4 & 2.509 & 0.656 & 0.302 & 0.027 & Chapman et al. (2005) \\
\hline SMMJ123606.85+621021.4 & 2.509 & 0.767 & 1.426 & 0.039 & Smail et al. (2004) \\
\hline SMMJ123606.85+621021.4 & 2.509 & 0.775 & 0.525 & 0.046 & Pope et al. (2006) \\
\hline SMMJ123606.85+621021.4 & 2.509 & 0.798 & 0.749 & 0.069 & Capak et al. (2004) \\
\hline SMMJ123606.85+621021.4 & 2.509 & 0.907 & 0.972 & 0.093 & Capak et al. (2004) \\
\hline SMMJ123606.85+621021.4 & 2.509 & 1.250 & 4.000 & 0.484 & Smail et al. (2004) \\
\hline SMMJ123606.85+621021.4 & 2.509 & 1.895 & 8.136 & 2.231 & Capak et al. (2004) \\
\hline SMMJ123606.85+621021.4 & 2.509 & 2.170 & 16.338 & 0.878 & Smail et al. (2004) \\
\hline
\end{tabular}


Table A.1. continued

\begin{tabular}{|c|c|c|c|c|c|}
\hline SMG & $z$ & $\begin{array}{r}\lambda_{\text {obs }} \\
(\mu \mathrm{m})\end{array}$ & $\begin{array}{r}\text { Flux } \\
(\mu \mathrm{Jy})\end{array}$ & $\begin{array}{l}\text { Error } \\
(\mu \mathrm{Jy})\end{array}$ & Reference \\
\hline SMMJ123606.85+621021.4 & 2.509 & 3.600 & 27.800 & 3.000 & Hainline (2008) \\
\hline SMMJ123606.85+621021.4 & 2.509 & 4.500 & 31.000 & 3.300 & Hainline (2008) \\
\hline SMMJ123606.85+621021.4 & 2.509 & 5.800 & 35.500 & 3.700 & Hainline (2008) \\
\hline SMMJ123606.85+621021.4 & 2.509 & 8.000 & 28.100 & 3.100 & Hainline (2008) \\
\hline SMMJ123606.85+621021.4 & 2.509 & 24.000 & 70.100 & 5.200 & Pope et al. (2006) \\
\hline SMMJ123606.85+621021.4 & 2.509 & 350.000 & 35100.000 & 6900.000 & Kovács et al. (2006) \\
\hline SMMJ123606.85+621021.4 & 2.509 & 850.000 & 11600.000 & 3500.000 & Chapman et al. (2005) \\
\hline SMMJ123606.85+621021.4 & 2.509 & 214000.000 & 74.400 & 4.100 & Chapman et al. (2005) \\
\hline SMMJ123616.15+621513.7 & 2.578 & 0.428 & 0.069 & 0.006 & Chapman et al. (2005); Pope et al. (2008) \\
\hline SMMJ123616.15+621513.7 & 2.578 & 0.443 & 0.084 & 0.019 & Capak et al. (2004) \\
\hline SMMJ123616.15+621513.7 & 2.578 & 0.547 & 0.134 & 0.027 & Capak et al. (2004) \\
\hline SMMJ123616.15+621513.7 & 2.578 & 0.653 & 0.260 & 0.025 & Capak et al. (2004) \\
\hline SMMJ123616.15+621513.7 & 2.578 & 0.573 & 0.076 & 0.007 & Chapman et al. (2003b) \\
\hline SMMJ123616.15+621513.7 & 2.578 & 0.656 & 0.191 & 0.017 & Chapman et al. (2005) \\
\hline SMMJ123616.15+621513.7 & 2.578 & 0.767 & 0.400 & 0.035 & Chapman et al. (2003b) \\
\hline SMMJ123616.15+621513.7 & 2.578 & 0.767 & 0.333 & 0.035 & Smail et al. (2004) \\
\hline SMMJ123616.15+621513.7 & 2.578 & 0.775 & 0.120 & 0.011 & Pope et al. (2006) \\
\hline SMMJ123616.15+621513.7 & 2.578 & 0.798 & 0.352 & 0.059 & Capak et al. (2004) \\
\hline SMMJ123616.15+621513.7 & 2.578 & 0.907 & 0.364 & 0.076 & Capak et al. (2004) \\
\hline SMMJ123616.15+621513.7 & 2.578 & 1.250 & 1.683 & 0.383 & Smail et al. (2004) \\
\hline SMMJ123616.15+621513.7 & 2.578 & 2.170 & 2.973 & 0.779 & Smail et al. (2004) \\
\hline SMMJ123616.15+621513.7 & 2.578 & 3.600 & 20.000 & 2.300 & Hainline (2008) \\
\hline SMMJ123616.15+621513.7 & 2.578 & 4.500 & 15.000 & 1.700 & Hainline (2008) \\
\hline SMMJ123616.15+621513.7 & 2.578 & 5.800 & 39.800 & 4.100 & Hainline (2008) \\
\hline SMMJ123616.15+621513.7 & 2.578 & 8.000 & 46.800 & 4.900 & Hainline (2008) \\
\hline SMMJ123616.15+621513.7 & 2.578 & 24.000 & 319.100 & 17.800 & Hainline (2008) \\
\hline SMMJ123616.15+621513.7 & 2.578 & 850.000 & 5800.000 & 1100.000 & Chapman et al. (2005) \\
\hline SMMJ123616.15+621513.7 & 2.578 & 214000.000 & 53.900 & 8.400 & Chapman et al. (2005) \\
\hline SMMJ123618.33+621550.5 & 1.865 & 0.365 & 0.223 & 0.032 & Capak et al. (2004); Pope et al. (2008) \\
\hline SMMJ123618.33+621550.5 & 1.865 & 0.428 & 0.145 & 0.013 & Chapman et al. (2005) \\
\hline SMMJ123618.33+621550.5 & 1.865 & 0.443 & 0.263 & 0.029 & Capak et al. (2004) \\
\hline SMMJ123618.33+621550.5 & 1.865 & 0.547 & 0.258 & 0.031 & Capak et al. (2004) \\
\hline SMMJ123618.33+621550.5 & 1.865 & 0.653 & 0.314 & 0.027 & Capak et al. (2004) \\
\hline SMMJ123618.33+621550.5 & 1.865 & 0.656 & 0.158 & 0.014 & Chapman et al. (2005) \\
\hline SMMJ123618.33+621550.5 & 1.865 & 0.767 & 0.248 & 0.022 & Chapman et al. (2003b) \\
\hline SMMJ123618.33+621550.5 & 1.865 & 0.767 & 0.345 & 0.042 & Smail et al. (2004) \\
\hline SMMJ123618.33+621550.5 & 1.865 & 0.798 & 0.444 & 0.065 & Capak et al. (2004) \\
\hline SMMJ123618.33+621550.5 & 1.865 & 0.907 & 0.559 & 0.086 & Capak et al. (2004) \\
\hline SMMJ123618.33+621550.5 & 1.865 & 1.250 & 2.061 & 0.424 & Smail et al. (2004) \\
\hline SMMJ123618.33+621550.5 & 1.865 & 2.170 & 3.992 & 0.879 & Smail et al. (2004) \\
\hline SMMJ123618.33+621550.5 & 1.865 & 3.600 & 15.000 & 1.900 & Hainline (2008) \\
\hline SMMJ123618.33+621550.5 & 1.865 & 4.500 & 19.900 & 2.200 & Hainline (2008) \\
\hline SMMJ123618.33+621550.5 & 1.865 & 5.800 & 27.300 & 2.800 & Hainline (2008) \\
\hline SMMJ123618.33+621550.5 & 1.865 & 8.000 & 20.000 & 2.200 & Hainline (2008) \\
\hline SMMJ123618.33+621550.5 & 1.865 & 24.000 & 343.800 & 18.800 & Hainline (2008) \\
\hline SMMJ123618.33+621550.5 & 1.865 & 850.000 & 7300.000 & 1100.000 & Chapman et al. (2005) \\
\hline SMMJ123618.33+621550.5 & 1.865 & 214000.000 & 151.000 & 11.000 & Chapman et al. (2005) \\
\hline SMMJ123621.27+621708.4 & 1.988 & 0.365 & 0.333 & 0.038 & Capak et al. (2004); Pope et al. (2008) \\
\hline SMMJ123621.27+621708.4 & 1.988 & 0.428 & 0.331 & 0.029 & Chapman et al. (2005) \\
\hline SMMJ123621.27+621708.4 & 1.988 & 0.443 & 0.472 & 0.044 & Capak et al. (2004) \\
\hline SMMJ123621.27+621708.4 & 1.988 & 0.547 & 0.522 & 0.036 & Capak et al. (2004) \\
\hline SMMJ123621.27+621708.4 & 1.988 & 0.653 & 0.622 & 0.033 & Capak et al. (2004) \\
\hline SMMJ123621.27+621708.4 & 1.988 & 0.656 & 0.398 & 0.035 & Chapman et al. (2005) \\
\hline SMMJ123621.27+621708.4 & 1.988 & 0.767 & 1.043 & 0.092 & Chapman et al. (2003b) \\
\hline SMMJ123621.27+621708.4 & 1.998 & 0.767 & 0.262 & 0.034 & Smail et al. (2004) \\
\hline SMMJ123621.27+621708.4 & 1.988 & 0.798 & 0.870 & 0.074 & Capak et al. (2004) \\
\hline SMMJ123621.27+621708.4 & 1.988 & 0.907 & 0.950 & 0.098 & Capak et al. (2004) \\
\hline SMMJ123621.27+621708.4 & 1.988 & 1.895 & 6.011 & 0.919 & Capak et al. (2004) \\
\hline SMMJ123621.27+621708.4 & 1.998 & 2.170 & 3.351 & 0.878 & Smail et al. (2004) \\
\hline SMMJ123621.27+621708.4 & 1.988 & 3.600 & 20.400 & 2.300 & Hainline (2008) \\
\hline SMMJ123621.27+621708.4 & 1.988 & 4.500 & 27.300 & 2.900 & Hainline (2008) \\
\hline SMMJ123621.27+621708.4 & 1.988 & 5.800 & 34.400 & 3.500 & Hainline (2008) \\
\hline SMMJ123621.27+621708.4 & 1.988 & 8.000 & 24.500 & 2.600 & Hainline (2008) \\
\hline SMMJ123621.27+621708.4 & 1.988 & 24.000 & 346.500 & 20.600 & Hainline (2008) \\
\hline SMMJ123621.27+621708.4 & 1.988 & 850.000 & 7800.000 & 1900.000 & Chapman et al. (2005) \\
\hline
\end{tabular}


Table A.1. continued

\begin{tabular}{|c|c|c|c|c|c|}
\hline SMG & $z$ & $\begin{array}{r}\lambda_{\mathrm{obs}} \\
(\mu \mathrm{m})\end{array}$ & $\begin{array}{r}\text { Flux } \\
(\mu \mathrm{Jy})\end{array}$ & $\begin{array}{l}\text { Error } \\
(\mu \mathrm{Jy})\end{array}$ & Reference \\
\hline SMMJ123621.27+621708.4 & 1.988 & 214000.000 & 148.000 & 11.000 & Chapman et al. (2005) \\
\hline SMMJ123622.65+621629.7 & 2.466 & 0.365 & 0.206 & 0.032 & Capak et al. (2004); Pope et al. (2008) \\
\hline SMMJ123622.65+621629.7 & 2.466 & 0.428 & 0.209 & 0.018 & Chapman et al. (2005) \\
\hline SMMJ123622.65+621629.7 & 2.466 & 0.443 & 0.310 & 0.039 & Capak et al. (2004) \\
\hline SMMJ123622.65+621629.7 & 2.466 & 0.547 & 0.348 & 0.034 & Capak et al. (2004) \\
\hline SMMJ123622.65+621629.7 & 2.466 & 0.573 & 0.283 & 0.025 & Chapman et al. (2003b) \\
\hline SMMJ123622.65+621629.7 & 2.466 & 0.653 & 0.416 & 0.029 & Capak et al. (2004) \\
\hline SMMJ123622.65+621629.7 & 2.466 & 0.656 & 0.251 & 0.022 & Chapman et al. (2005) \\
\hline SMMJ123622.65+621629.7 & 2.466 & 0.767 & 0.455 & 0.040 & Chapman et al. (2003b) \\
\hline SMMJ123622.65+621629.7 & 2.466 & 0.767 & 0.477 & 0.042 & Smail et al. (2004) \\
\hline SMMJ123622.65+621629.7 & 2.466 & 2.170 & 5.072 & 0.930 & Smail et al. (2004) \\
\hline SMMJ123622.65+621629.7 & 2.466 & 3.600 & 19.800 & 2.200 & Hainline (2008) \\
\hline SMMJ123622.65+621629.7 & 2.466 & 4.500 & 27.600 & 2.900 & Hainline (2008) \\
\hline SMMJ123622.65+621629.7 & 2.466 & 5.800 & 35.300 & 3.600 & Hainline (2008) \\
\hline SMMJ123622.65+621629.7 & 2.466 & 8.000 & 26.400 & 2.800 & Hainline (2008) \\
\hline SMMJ123622.65+621629.7 & 2.466 & 24.000 & 368.500 & 20.700 & Hainline (2008) \\
\hline SMMJ123622.65+621629.7 & 2.466 & 850.000 & 7700.000 & 1300.000 & Chapman et al. (2005) \\
\hline SMMJ123622.65+621629.7 & 2.466 & 214000.000 & 70.900 & 8.700 & Chapman et al. (2005) \\
\hline SMMJ123629.13+621045.8 & 1.013 & 0.365 & 0.130 & 0.030 & Capak et al. (2004) \\
\hline SMMJ123629.13+621045.8 & 1.013 & 0.428 & 0.132 & 0.012 & Chapman et al. (2005) \\
\hline SMMJ123629.13+621045.8 & 1.013 & 0.443 & 0.191 & 0.027 & Capak et al. (2004) \\
\hline SMMJ123629.13+621045.8 & 1.013 & 0.547 & 0.386 & 0.033 & Capak et al. (2004) \\
\hline SMMJ123629.13+621045.8 & 1.013 & 0.653 & 0.872 & 0.034 & Capak et al. (2004) \\
\hline SMMJ123629.13+621045.8 & 1.013 & 0.656 & 0.525 & 0.046 & Chapman et al. (2005) \\
\hline SMMJ123629.13+621045.8 & 1.013 & 0.767 & 2.794 & 0.026 & Smail et al. (2004) \\
\hline SMMJ123629.13+621045.8 & 1.013 & 0.775 & 2.754 & 0.242 & Pope et al. (2006) \\
\hline SMMJ123629.13+621045.8 & 1.013 & 0.798 & 2.533 & 0.103 & Capak et al. (2004) \\
\hline SMMJ123629.13+621045.8 & 1.013 & 0.907 & 4.144 & 0.150 & Capak et al. (2004) \\
\hline SMMJ123629.13+621045.8 & 1.013 & 1.250 & 11.222 & 0.505 & Smail et al. (2004) \\
\hline SMMJ123629.13+621045.8 & 1.013 & 1.895 & 23.795 & 1.529 & Capak et al. (2004) \\
\hline SMMJ123629.13+621045.8 & 1.013 & 2.170 & 40.290 & 0.735 & Smail et al. (2004) \\
\hline SMMJ123629.13+621045.8 & 1.013 & 3.600 & 93.100 & 9.600 & Hainline (2008) \\
\hline SMMJ123629.13+621045.8 & 1.013 & 4.500 & 87.000 & 9.000 & Hainline (2008) \\
\hline SMMJ123629.13+621045.8 & 1.013 & 5.800 & 75.800 & 7.700 & Hainline (2008) \\
\hline SMMJ123629.13+621045.8 & 1.013 & 8.000 & 72.000 & 7.400 & Hainline (2008) \\
\hline SMMJ123629.13+621045.8 & 1.013 & 24.000 & 664.800 & 34.200 & Hainline (2008) \\
\hline SMMJ123629.13+621045.8 & 1.013 & 850.000 & 5000.000 & 1300.000 & Chapman et al. (2005) \\
\hline SMMJ123629.13+621045.8 & 1.013 & 214000.000 & 81.400 & 8.700 & Chapman et al. (2005) \\
\hline SMMJ123632.61+620800.1 & 1.993 & 0.365 & 1.791 & 0.084 & Capak et al. (2004) \\
\hline SMMJ123632.61+620800.1 & 1.993 & 0.428 & 1.096 & 0.096 & Chapman et al. (2005) \\
\hline SMMJ123632.61+620800.1 & 1.993 & 0.443 & 1.078 & 0.059 & Capak et al. (2004) \\
\hline SMMJ123632.61+620800.1 & 1.993 & 0.547 & 0.990 & 0.044 & Capak et al. (2004) \\
\hline SMMJ123632.61+620800.1 & 1.993 & 0.653 & 1.158 & 0.039 & Capak et al. (2004) \\
\hline SMMJ123632.61+620800.1 & 1.993 & 0.656 & 1.318 & 0.116 & Chapman et al. (2005) \\
\hline SMMJ123632.61+620800.1 & 1.993 & 0.767 & 2.179 & 0.059 & Smail et al. (2004) \\
\hline SMMJ123632.61+620800.1 & 1.993 & 0.798 & 2.001 & 0.103 & Capak et al. (2004) \\
\hline SMMJ123632.61+620800.1 & 1.993 & 0.907 & 2.398 & 0.140 & Capak et al. (2004) \\
\hline SMMJ123632.61+620800.1 & 1.993 & 1.250 & 5.625 & 0.859 & Smail et al. (2004) \\
\hline SMMJ123632.61+620800.1 & 1.993 & 1.895 & 6.511 & 1.837 & Capak et al. (2004) \\
\hline SMMJ123632.61+620800.1 & 1.993 & 2.170 & 5.665 & 0.865 & Smail et al. (2004) \\
\hline SMMJ123632.61+620800.1 & 1.993 & 3.600 & 21.200 & 2.500 & Hainline (2008) \\
\hline SMMJ123632.61+620800.1 & 1.993 & 4.500 & 27.800 & 3.000 & Hainline (2008) \\
\hline SMMJ123632.61+620800.1 & 1.993 & 5.800 & 50.700 & 5.200 & Hainline (2008) \\
\hline SMMJ123632.61+620800.1 & 1.993 & 8.000 & 114.300 & 11.700 & Hainline (2008) \\
\hline SMMJ123632.61+620800.1 & 1.993 & 24.000 & 849.000 & 43.300 & Hainline (2008) \\
\hline SMMJ123632.61+620800.1 & 1.993 & 850.000 & 5500.000 & 1300.000 & Chapman et al. (2005) \\
\hline SMMJ123632.61+620800.1 & 1.993 & 214000.000 & 90.600 & 9.300 & Chapman et al. (2005) \\
\hline SMMJ123634.51+621241.0 & 1.219 & 0.365 & 0.671 & 0.052 & Capak et al. (2004) \\
\hline SMMJ123634.51+621241.0 & 1.219 & 0.428 & 0.631 & 0.056 & Chapman et al. (2005); Pope et al. (2008) \\
\hline SMMJ123634.51+621241.0 & 1.219 & 0.443 & 0.830 & 0.056 & Capak et al. (2004) \\
\hline SMMJ123634.51+621241.0 & 1.219 & 0.547 & 0.960 & 0.043 & Capak et al. (2004) \\
\hline SMMJ123634.51+621241.0 & 1.219 & 0.653 & 1.538 & 0.042 & Capak et al. (2004) \\
\hline SMMJ123634.51+621241.0 & 1.219 & 0.656 & 1.000 & 0.088 & Chapman et al. (2005) \\
\hline SMMJ123634.51+621241.0 & 1.219 & 0.767 & 3.149 & 0.057 & Smail et al. (2004) \\
\hline
\end{tabular}


Table A.1. continued

\begin{tabular}{|c|c|c|c|c|c|}
\hline SMG & $z$ & $\begin{array}{r}\lambda_{\mathrm{obs}} \\
(\mu \mathrm{m})\end{array}$ & $\begin{array}{c}\text { Flux } \\
(\mu \mathrm{Jy})\end{array}$ & $\begin{array}{l}\text { Error } \\
(\mu \mathrm{J} y)\end{array}$ & Reference \\
\hline SMMJ123634.51+621241.0 & 1.219 & 0.775 & 3.020 & 0.266 & Pope et al. (2006) \\
\hline SMMJ123634.51+621241.0 & 1.219 & 0.798 & 2.914 & 0.109 & Capak et al. (2004) \\
\hline SMMJ123634.51+621241.0 & 1.219 & 0.907 & 4.834 & 0.164 & Capak et al. (2004) \\
\hline SMMJ123634.51+621241.0 & 1.219 & 1.250 & 8.914 & 0.322 & Smail et al. (2004) \\
\hline SMMJ123634.51+621241.0 & 1.219 & 1.895 & 17.259 & 1.030 & Capak et al. (2004) \\
\hline SMMJ123634.51+621241.0 & 1.219 & 2.170 & 25.894 & 0.706 & Smail et al. (2004) \\
\hline SMMJ123634.51+621241.0 & 1.219 & 3.600 & 64.400 & 6.700 & Hainline (2008) \\
\hline SMMJ123634.51+621241.0 & 1.219 & 4.500 & 72.500 & 7.400 & Hainline (2008) \\
\hline SMMJ123634.51+621241.0 & 1.219 & 5.800 & 59.200 & 6.000 & Hainline (2008) \\
\hline SMMJ123634.51+621241.0 & 1.219 & 8.000 & 73.600 & 7.500 & Hainline (2008) \\
\hline SMMJ123634.51+621241.0 & 1.219 & 24.000 & 464.600 & 23.800 & Hainline (2008) \\
\hline SMMJ123634.51+621241.0 & 1.219 & 70.000 & 13900.000 & 2100.000 & Hainline (2008) \\
\hline SMMJ123634.51+621241.0 & 1.219 & 160.000 & 110000.000 & 27000.000 & Huynh et al. (2007) \\
\hline SMMJ123634.51+621241.0 & 1.219 & 850.000 & 4300.000 & 1400.000 & Chapman et al. (2005) \\
\hline SMMJ123634.51+621241.0 & 1.219 & 214000.000 & 230.000 & 13.800 & Chapman et al. (2005) \\
\hline SMMJ123635.59+621424.1 & 2.005 & 0.365 & 0.965 & 0.061 & Capak et al. (2004) \\
\hline SMMJ123635.59+621424.1 & 2.005 & 0.428 & 0.759 & 0.067 & Chapman et al. (2005) \\
\hline SMMJ123635.59+621424.1 & 2.005 & 0.443 & 0.861 & 0.056 & Capak et al. (2004) \\
\hline SMMJ123635.59+621424.1 & 2.005 & 0.547 & 0.950 & 0.046 & Capak et al. (2004) \\
\hline SMMJ123635.59+621424.1 & 2.005 & 0.653 & 1.107 & 0.036 & Capak et al. (2004) \\
\hline SMMJ123635.59+621424.1 & 2.005 & 0.656 & 0.759 & 0.067 & Chapman et al. (2005) \\
\hline SMMJ123635.59+621424.1 & 2.005 & 0.767 & 1.637 & 0.045 & Smail et al. (2004) \\
\hline SMMJ123635.59+621424.1 & 2.005 & 0.798 & 1.643 & 0.092 & Capak et al. (2004) \\
\hline SMMJ123635.59+621424.1 & 2.005 & 0.907 & 2.065 & 0.120 & Capak et al. (2004) \\
\hline SMMJ123635.59+621424.1 & 2.005 & 1.250 & 5.421 & 0.338 & Smail et al. (2004) \\
\hline SMMJ123635.59+621424.1 & 2.005 & 1.895 & 13.777 & 0.725 & Capak et al. (2004) \\
\hline SMMJ123635.59+621424.1 & 2.005 & 2.170 & 19.107 & 0.691 & Smail et al. (2004) \\
\hline SMMJ123635.59+621424.1 & 2.005 & 3.600 & 65.000 & 6.700 & Hainline (2008) \\
\hline SMMJ123635.59+621424.1 & 2.005 & 4.500 & 100.000 & 10.100 & Hainline (2008) \\
\hline SMMJ123635.59+621424.1 & 2.005 & 5.800 & 175.800 & 17.600 & Hainline (2008) \\
\hline SMMJ123635.59+621424.1 & 2.005 & 8.000 & 300.500 & 30.200 & Hainline (2008) \\
\hline SMMJ123635.59+621424.1 & 2.005 & 24.000 & 1445.100 & 72.800 & Hainline (2008) \\
\hline SMMJ123635.59+621424.1 & 2.005 & 850.000 & 5500.000 & 1400.000 & Chapman et al. (2005) \\
\hline SMMJ123635.59+621424.1 & 2.005 & 214000.000 & 87.800 & 8.800 & Chapman et al. (2005) \\
\hline SMMJ123636.75+621156.1 & 0.557 & 0.365 & 0.707 & 0.052 & Capak et al. (2004) \\
\hline SMMJ123636.75+621156.1 & 0.557 & 0.428 & 6.310 & 0.555 & Chapman et al. (2005) \\
\hline SMMJ123636.75+621156.1 & 0.557 & 0.443 & 1.539 & 0.075 & Capak et al. (2004) \\
\hline SMMJ123636.75+621156.1 & 0.557 & 0.547 & 2.183 & 0.061 & Capak et al. (2004) \\
\hline SMMJ123636.75+621156.1 & 0.557 & 0.653 & 4.021 & 0.059 & Capak et al. (2004) \\
\hline SMMJ123636.75+621156.1 & 0.557 & 0.656 & 8.318 & 0.732 & Chapman et al. (2005) \\
\hline SMMJ123636.75+621156.1 & 0.557 & 0.767 & 6.519 & 0.060 & Smail et al. (2004) \\
\hline SMMJ123636.75+621156.1 & 0.557 & 0.798 & 6.661 & 0.152 & Capak et al. (2004) \\
\hline SMMJ123636.75+621156.1 & 0.557 & 0.907 & 8.273 & 0.199 & Capak et al. (2004) \\
\hline SMMJ123636.75+621156.1 & 0.557 & 1.250 & 12.885 & 0.466 & Smail et al. (2004) \\
\hline SMMJ123636.75+621156.1 & 0.557 & 1.895 & 17.872 & 0.773 & Capak et al. (2004) \\
\hline SMMJ123636.75+621156.1 & 0.557 & 2.170 & 23.184 & 0.632 & Smail et al. (2004) \\
\hline SMMJ123636.75+621156.1 & 0.557 & 3.600 & 22.600 & 2.500 & Hainline (2008) \\
\hline SMMJ123636.75+621156.1 & 0.557 & 4.500 & 16.200 & 1.800 & Hainline (2008) \\
\hline SMMJ123636.75+621156.1 & 0.557 & 5.800 & 14.400 & 1.500 & Hainline (2008) \\
\hline SMMJ123636.75+621156.1 & 0.557 & 8.000 & 13.400 & 1.600 & Hainline (2008) \\
\hline SMMJ123636.75+621156.1 & 0.557 & 850.000 & 7000.000 & 2100.000 & Chapman et al. (2005) \\
\hline SMMJ123636.75+621156.1 & 0.557 & 214000.000 & 39.000 & 8.000 & Chapman et al. (2005) \\
\hline SMMJ123651.76+621221.3 & 0.298 & 0.365 & 0.786 & 0.055 & Capak et al. (2004) \\
\hline SMMJ123651.76+621221.3 & 0.298 & 0.428 & 8.318 & 0.732 & Chapman et al. (2005) \\
\hline SMMJ123651.76+621221.3 & 0.298 & 0.443 & 1.427 & 0.077 & Capak et al. (2004) \\
\hline SMMJ123651.76+621221.3 & 0.298 & 0.547 & 3.485 & 0.073 & Capak et al. (2004) \\
\hline SMMJ123651.76+621221.3 & 0.298 & 0.653 & 5.615 & 0.071 & Capak et al. (2004) \\
\hline SMMJ123651.76+621221.3 & 0.298 & 0.656 & 10.000 & 0.880 & Chapman et al. (2005) \\
\hline SMMJ123651.76+621221.3 & 0.298 & 0.798 & 8.325 & 0.166 & Capak et al. (2004) \\
\hline SMMJ123651.76+621221.3 & 0.298 & 0.907 & 9.895 & 0.220 & Capak et al. (2004) \\
\hline SMMJ123651.76+621221.3 & 0.298 & 1.895 & 20.758 & 0.893 & Capak et al. (2004) \\
\hline SMMJ123651.76+621221.3 & 0.298 & 3.600 & 21.100 & 2.300 & Hainline (2008) \\
\hline SMMJ123651.76+621221.3 & 0.298 & 4.500 & 21.100 & 2.200 & Hainline (2008) \\
\hline SMMJ123651.76+621221.3 & 0.298 & 5.800 & 17.700 & 1.800 & Hainline (2008) \\
\hline SMMJ123651.76+621221.3 & 0.298 & 8.000 & 46.900 & 4.800 & Hainline (2008) \\
\hline
\end{tabular}


Table A.1. continued

\begin{tabular}{|c|c|c|c|c|c|}
\hline SMG & $z$ & $\begin{array}{r}\lambda_{\mathrm{obs}} \\
(\mu \mathrm{m})\end{array}$ & $\begin{array}{c}\text { Flux } \\
(\mu \mathrm{Jy})\end{array}$ & $\begin{array}{l}\text { Error } \\
(\mu \mathrm{Jy})\end{array}$ & Reference \\
\hline SMMJ123651.76+621221.3 & 0.298 & 24.000 & 107.000 & 9.900 & Hainline (2008) \\
\hline SMMJ123651.76+621221.3 & 0.298 & 850.000 & 4600.000 & 800.000 & Chapman et al. (2005) \\
\hline SMMJ123651.76+621221.3 & 0.298 & 214000.000 & 49.300 & 7.900 & Chapman et al. (2005) \\
\hline SMMJ123707.21+621408.1 & 2.484 & 0.428 & 0.063 & 0.006 & Chapman et al. (2005); Pope et al. (2008) \\
\hline SMMJ123707.21+621408.1 & 2.484 & 0.443 & 0.071 & 0.018 & Capak et al. (2004) \\
\hline SMMJ123707.21+621408.1 & 2.484 & 0.547 & 0.108 & 0.029 & Capak et al. (2004) \\
\hline SMMJ123707.21+621408.1 & 2.484 & 0.653 & 0.203 & 0.027 & Capak et al. (2004) \\
\hline SMMJ123707.21+621408.1 & 2.484 & 0.656 & 0.145 & 0.013 & Chapman et al. (2005) \\
\hline SMMJ123707.21+621408.1 & 2.484 & 0.767 & 0.368 & 0.035 & Smail et al. (2004) \\
\hline SMMJ123707.21+621408.1 & 2.484 & 0.775 & 0.251 & 0.022 & Pope et al. (2006) \\
\hline SMMJ123707.21+621408.1 & 2.484 & 0.798 & 0.228 & 0.062 & Capak et al. (2004) \\
\hline SMMJ123707.21+621408.1 & 2.484 & 0.907 & 0.484 & 0.086 & Capak et al. (2004) \\
\hline SMMJ123707.21+621408.1 & 2.484 & 1.250 & 1.593 & 0.407 & Smail et al. (2004) \\
\hline SMMJ123707.21+621408.1 & 2.484 & 1.895 & 4.146 & 1.065 & Capak et al. (2004) \\
\hline SMMJ123707.21+621408.1 & 2.484 & 2.170 & 6.269 & 0.604 & Smail et al. (2004) \\
\hline SMMJ123707.21+621408.1 & 2.484 & 3.600 & 19.400 & 2.100 & Hainline (2008) \\
\hline SMMJ123707.21+621408.1 & 2.484 & 4.500 & 26.300 & 2.800 & Hainline (2008) \\
\hline SMMJ123707.21+621408.1 & 2.484 & 5.800 & 36.000 & 3.700 & Hainline (2008) \\
\hline SMMJ123707.21+621408.1 & 2.484 & 8.000 & 28.200 & 2.900 & Hainline (2008) \\
\hline SMMJ123707.21+621408.1 & 2.484 & 24.000 & 232.800 & 15.100 & Hainline (2008) \\
\hline SMMJ123707.21+621408.1 & 2.484 & 850.000 & 4700.000 & 1500.000 & Chapman et al. (2005) \\
\hline SMMJ123707.21+621408.1 & 2.484 & 214000.000 & 45.000 & 7.900 & Chapman et al. (2005) \\
\hline SMMJ123711.98+621325.7 & 1.992 & 0.365 & 0.130 & 0.031 & Capak et al. (2004); Pope et al. (2008) \\
\hline SMMJ123711.98+621325.7 & 1.992 & 0.428 & 0.145 & 0.013 & Chapman et al. (2005) \\
\hline SMMJ123711.98+621325.7 & 1.992 & 0.443 & 0.181 & 0.024 & Capak et al. (2004) \\
\hline SMMJ123711.98+621325.7 & 1.992 & 0.547 & 0.195 & 0.028 & Capak et al. (2004) \\
\hline SMMJ123711.98+621325.7 & 1.992 & 0.653 & 0.199 & 0.025 & Capak et al. (2004) \\
\hline SMMJ123711.98+621325.7 & 1.992 & 0.656 & 0.174 & 0.015 & Chapman et al. (2005) \\
\hline SMMJ123711.98+621325.7 & 1.992 & 0.767 & 0.372 & 0.036 & Smail et al. (2004) \\
\hline SMMJ123711.98+621325.7 & 1.992 & 0.798 & 0.365 & 0.061 & Capak et al. (2004) \\
\hline SMMJ123711.98+621325.7 & 1.992 & 0.907 & 0.344 & 0.076 & Capak et al. (2004) \\
\hline SMMJ123711.98+621325.7 & 1.992 & 2.170 & 2.194 & 0.575 & Smail et al. (2004) \\
\hline SMMJ123711.98+621325.7 & 1.992 & 3.600 & 9.300 & 1.100 & Hainline (2008) \\
\hline SMMJ123711.98+621325.7 & 1.992 & 4.500 & 11.500 & 1.300 & Hainline (2008) \\
\hline SMMJ123711.98+621325.7 & 1.992 & 5.800 & 15.700 & 1.600 & Hainline (2008) \\
\hline SMMJ123711.98+621325.7 & 1.992 & 8.000 & 12.100 & 1.300 & Hainline (2008) \\
\hline SMMJ123711.98+621325.7 & 1.992 & 24.000 & 250.700 & 15.200 & Hainline (2008) \\
\hline SMMJ123711.98+621325.7 & 1.992 & 850.000 & 4200.000 & 1400.000 & Chapman et al. (2005) \\
\hline SMMJ123711.98+621325.7 & 1.992 & 214000.000 & 53.900 & 8.100 & Chapman et al. (2005) \\
\hline SMMJ123712.05+621212.3 & 2.914 & 0.428 & 0.058 & 0.005 & Chapman et al. (2005) \\
\hline SMMJ123712.05+621212.3 & 2.914 & 0.443 & 0.112 & 0.021 & Capak et al. (2004) \\
\hline SMMJ123712.05+621212.3 & 2.914 & 0.547 & 0.098 & 0.027 & Capak et al. (2004) \\
\hline SMMJ123712.05+621212.3 & 2.914 & 0.653 & 0.135 & 0.025 & Capak et al. (2004) \\
\hline SMMJ123712.05+621212.3 & 2.914 & 0.656 & 0.229 & 0.020 & Chapman et al. (2005) \\
\hline SMMJ123712.05+621212.3 & 2.914 & 0.767 & 0.127 & 0.033 & Smail et al. (2004) \\
\hline SMMJ123712.05+621212.3 & 2.914 & 2.170 & 3.260 & 0.832 & Smail et al. (2004) \\
\hline SMMJ123712.05+621212.3 & 2.914 & 5.800 & 20.800 & 2.100 & Hainline (2008) \\
\hline SMMJ123712.05+621212.3 & 2.914 & 8.000 & 23.000 & 2.400 & Hainline (2008) \\
\hline SMMJ123712.05+621212.3 & 2.914 & 24.000 & 31.000 & 8.200 & Hainline (2008) \\
\hline SMMJ123712.05+621212.3 & 2.914 & 850.000 & 8000.000 & 1800.000 & Chapman et al. (2005) \\
\hline SMMJ123712.05+621212.3 & 2.914 & 214000.000 & 21.000 & 4.000 & Chapman et al. (2005) \\
\hline SMMJ123716.01+620323.3 & 2.037 & 0.365 & 41.166 & 0.396 & Capak et al. (2004) \\
\hline SMMJ123716.01+620323.3 & 2.037 & 0.428 & 27.542 & 2.423 & Chapman et al. (2005) \\
\hline SMMJ123716.01+620323.3 & 2.037 & 0.443 & 32.085 & 0.293 & Capak et al. (2004) \\
\hline SMMJ123716.01+620323.3 & 2.037 & 0.547 & 38.408 & 0.166 & Capak et al. (2004) \\
\hline SMMJ123716.01+620323.3 & 2.037 & 0.653 & 40.187 & 0.171 & Capak et al. (2004) \\
\hline SMMJ123716.01+620323.3 & 2.037 & 0.656 & 30.199 & 2.657 & Chapman et al. (2005) \\
\hline SMMJ123716.01+620323.3 & 2.037 & 0.767 & 54.724 & 0.502 & Smail et al. (2004) \\
\hline SMMJ123716.01+620323.3 & 2.037 & 0.798 & 58.598 & 0.555 & Capak et al. (2004) \\
\hline SMMJ123716.01+620323.3 & 2.037 & 0.907 & 66.554 & 0.586 & Capak et al. (2004) \\
\hline SMMJ123716.01+620323.3 & 2.037 & 1.895 & 93.546 & 1.694 & Capak et al. (2004) \\
\hline SMMJ123716.01+620323.3 & 2.037 & 2.170 & 377.742 & 33.237 & Smail et al. (2004) \\
\hline SMMJ123716.01+620323.3 & 2.037 & 850.000 & 5300.000 & 1700.000 & Chapman et al. (2005) \\
\hline
\end{tabular}


Table A.1. continued

\begin{tabular}{|c|c|c|c|c|c|}
\hline SMG & $z$ & $\begin{array}{r}\lambda_{\mathrm{obs}} \\
(\mu \mathrm{m})\end{array}$ & $\begin{array}{r}\text { Flux } \\
(\mu \mathrm{Jy})\end{array}$ & $\begin{array}{l}\text { Error } \\
(\mu \mathrm{Jy})\end{array}$ & Reference \\
\hline SMMJ123716.01+620323.3 & 2.037 & 214000.000 & 109.000 & 11.400 & Chapman et al. (2005) \\
\hline SMMJ123721.87+621035.3 & 0.979 & 0.365 & 0.768 & 0.058 & Capak et al. (2004); Menéndez-Delmestre et al. (2009) \\
\hline SMMJ123721.87+621035.3 & 0.979 & 0.428 & 0.692 & 0.061 & Chapman et al. (2005) \\
\hline SMMJ123721.87+621035.3 & 0.979 & 0.443 & 0.911 & 0.057 & Capak et al. (2004) \\
\hline SMMJ123721.87+621035.3 & 0.979 & 0.547 & 1.476 & 0.060 & Capak et al. (2004) \\
\hline SMMJ123721.87+621035.3 & 0.979 & 0.653 & 2.734 & 0.051 & Capak et al. (2004) \\
\hline SMMJ123721.87+621035.3 & 0.979 & 0.656 & 1.738 & 0.153 & Chapman et al. (2005) \\
\hline SMMJ123721.87+621035.3 & 0.979 & 0.767 & 7.017 & 0.064 & Smail et al. (2004) \\
\hline SMMJ123721.87+621035.3 & 0.979 & 0.798 & 6.688 & 0.171 & Capak et al. (2004) \\
\hline SMMJ123721.87+621035.3 & 0.979 & 0.907 & 10.484 & 0.234 & Capak et al. (2004) \\
\hline SMMJ123721.87+621035.3 & 0.979 & 1.895 & 35.627 & 1.992 & Capak et al. (2004) \\
\hline SMMJ123721.87+621035.3 & 0.979 & 2.170 & 45.415 & 1.238 & Smail et al. (2004) \\
\hline SMMJ123721.87+621035.3 & 0.979 & 3.600 & 70.900 & 7.300 & Hainline (2008) \\
\hline SMMJ123721.87+621035.3 & 0.979 & 4.500 & 53.900 & 5.600 & Hainline (2008) \\
\hline SMMJ123721.87+621035.3 & 0.979 & 5.800 & 43.800 & 4.400 & Hainline (2008) \\
\hline SMMJ123721.87+621035.3 & 0.979 & 8.000 & 44.300 & 4.600 & Hainline (2008) \\
\hline SMMJ123721.87+621035.3 & 0.979 & 24.000 & 245.600 & 15.900 & Hainline (2008) \\
\hline SMMJ123721.87+621035.3 & 0.979 & 850.000 & 12000.000 & 3900.000 & Chapman et al. (2005) \\
\hline SMMJ123721.87+621035.3 & 0.979 & 214000.000 & 41.000 & 9.000 & Chapman et al. (2005) \\
\hline SMMJ131201.17+424208.1 & 3.405 & 0.428 & 0.302 & 0.027 & Chapman et al. (2005) \\
\hline SMMJ131201.17+424208.1 & 3.405 & 0.630 & 0.649 & 0.057 & Fomalont et al. (2006) \\
\hline SMMJ131201.17+424208.1 & 3.405 & 0.656 & 0.912 & 0.080 & Chapman et al. (2005) \\
\hline SMMJ131201.17+424208.1 & 3.405 & 0.767 & 1.400 & 0.075 & Smail et al. (2004) \\
\hline SMMJ131201.17+424208.1 & 3.405 & 0.920 & 1.535 & 0.135 & Fomalont et al. (2006) \\
\hline SMMJ131201.17+424208.1 & 3.405 & 1.250 & 2.138 & 0.455 & Smail et al. (2004) \\
\hline SMMJ131201.17+424208.1 & 3.405 & 2.170 & 4.258 & 0.749 & Smail et al. (2004) \\
\hline SMMJ131201.17+424208.1 & 3.405 & 3.600 & 10.600 & 1.100 & Hainline (2008) \\
\hline SMMJ131201.17+424208.1 & 3.405 & 4.500 & 15.100 & 1.600 & Hainline (2008) \\
\hline SMMJ131201.17+424208.1 & 3.405 & 5.800 & 24.100 & 3.700 & Hainline (2008) \\
\hline SMMJ131201.17+424208.1 & 3.405 & 8.000 & 29.300 & 4.100 & Hainline (2008) \\
\hline SMMJ131201.17+424208.1 & 3.405 & 350.000 & 21100.000 & 7700.000 & Kovács et al. (2006) \\
\hline SMMJ131201.17+424208.1 & 3.405 & 850.000 & 6200.000 & 1200.000 & Chapman et al. (2005) \\
\hline SMMJ131201.17+424208.1 & 3.405 & 214000.000 & 49.100 & 6.000 & Chapman et al. (2005) \\
\hline SMMJ131208.82+424129.1 & 1.544 & 0.428 & 0.191 & 0.017 & Chapman et al. (2005) \\
\hline SMMJ131208.82+424129.1 & 1.544 & 0.630 & 0.409 & 0.036 & Fomalont et al. (2006) \\
\hline SMMJ131208.82+424129.1 & 1.544 & 0.656 & 1.000 & 0.088 & Chapman et al. (2005) \\
\hline SMMJ131208.82+424129.1 & 1.544 & 0.767 & 1.337 & 0.012 & Smail et al. (2004) \\
\hline SMMJ131208.82+424129.1 & 1.544 & 0.920 & 1.165 & 0.102 & Fomalont et al. (2006) \\
\hline SMMJ131208.82+424129.1 & 1.544 & 1.250 & 5.371 & 0.693 & Smail et al. (2004) \\
\hline SMMJ131208.82+424129.1 & 1.544 & 2.170 & 11.620 & 1.022 & Smail et al. (2004) \\
\hline SMMJ131208.82+424129.1 & 1.544 & 3.600 & 27.300 & 2.800 & Hainline (2008) \\
\hline SMMJ131208.82+424129.1 & 1.544 & 4.500 & 37.300 & 3.800 & Hainline (2008) \\
\hline SMMJ131208.82+424129.1 & 1.544 & 5.800 & 33.400 & 4.400 & Hainline (2008) \\
\hline SMMJ131208.82+424129.1 & 1.544 & 8.000 & 26.900 & 4.000 & Hainline (2008) \\
\hline SMMJ131208.82+424129.1 & 1.544 & 850.000 & 4900.000 & 1500.000 & Chapman et al. (2005) \\
\hline SMMJ131208.82+424129.1 & 1.544 & 214000.000 & 82.400 & 4.800 & Chapman et al. (2005) \\
\hline SMMJ131212.69+424422.5 & 2.805 & 0.656 & 0.076 & 0.007 & Chapman et al. (2005) \\
\hline SMMJ131212.69+424422.5 & 2.805 & 2.170 & 5.026 & 0.959 & Smail et al. (2004) \\
\hline SMMJ131212.69+424422.5 & 2.805 & 3.600 & 4.600 & 0.600 & Hainline (2008) \\
\hline SMMJ131212.69+424422.5 & 2.805 & 4.500 & 7.200 & 1.000 & Hainline (2008) \\
\hline SMMJ131212.69+424422.5 & 2.805 & 850.000 & 5600.000 & 1900.000 & Chapman et al. (2005) \\
\hline SMMJ131212.69+424422.5 & 2.805 & 214000.000 & 102.600 & 7.400 & Chapman et al. (2005) \\
\hline SMMJ131215.27+423900.9 & 2.565 & 0.428 & 131.826 & 11.599 & Chapman et al. (2005) \\
\hline SMMJ131215.27+423900.9 & 2.565 & 0.630 & 135.521 & 11.925 & Fomalont et al. (2006) \\
\hline SMMJ131215.27+423900.9 & 2.565 & 0.656 & 173.780 & 15.291 & Chapman et al. (2005) \\
\hline SMMJ131215.27+423900.9 & 2.565 & 0.767 & 157.826 & 1.447 & Smail et al. (2004) \\
\hline SMMJ131215.27+423900.9 & 2.565 & 0.920 & 140.016 & 12.320 & Fomalont et al. (2006) \\
\hline SMMJ131215.27+423900.9 & 2.565 & 1.250 & 215.818 & 1.978 & Smail et al. (2004) \\
\hline SMMJ131215.27+423900.9 & 2.565 & 2.170 & 211.444 & 1.939 & Smail et al. (2004) \\
\hline SMMJ131215.27+423900.9 & 2.565 & 3.600 & 249.900 & 25.100 & Hainline (2008) \\
\hline SMMJ131215.27+423900.9 & 2.565 & 4.500 & 296.500 & 29.700 & Hainline (2008) \\
\hline SMMJ131215.27+423900.9 & 2.565 & 5.800 & 481.100 & 53.500 & Hainline (2008) \\
\hline
\end{tabular}


Table A.1. continued

\begin{tabular}{|c|c|c|c|c|c|}
\hline SMG & $z$ & $\begin{array}{r}\lambda_{\mathrm{obs}} \\
(\mu \mathrm{m})\end{array}$ & $\begin{array}{c}\text { Flux } \\
(\mu \mathrm{Jy})\end{array}$ & $\begin{array}{l}\text { Error } \\
(\mu \mathrm{Jy})\end{array}$ & Reference \\
\hline SMMJ131215.27+423900.9 & 2.565 & 8.000 & 831.400 & 85.100 & Hainline (2008) \\
\hline SMMJ131215.27+423900.9 & 2.565 & 850.000 & 4400.000 & 1000.000 & Chapman et al. (2005) \\
\hline SMMJ131215.27+423900.9 & 2.565 & 214000.000 & 69.300 & 4.000 & Chapman et al. (2005) \\
\hline SMMJ131222.35+423814.1 & 2.565 & 0.428 & 6.918 & 0.609 & Chapman et al. (2005) \\
\hline SMMJ131222.35+423814.1 & 2.565 & 0.630 & 12.360 & 1.088 & Fomalont et al. (2006) \\
\hline SMMJ131222.35+423814.1 & 2.565 & 0.656 & 30.199 & 2.657 & Chapman et al. (2005) \\
\hline SMMJ131222.35+423814.1 & 2.565 & 0.767 & 27.937 & 0.256 & Smail et al. (2004) \\
\hline SMMJ131222.35+423814.1 & 2.565 & 0.920 & 24.332 & 2.141 & Fomalont et al. (2006) \\
\hline SMMJ131222.35+423814.1 & 2.565 & 1.250 & 39.636 & 1.080 & Smail et al. (2004) \\
\hline SMMJ131222.35+423814.1 & 2.565 & 2.170 & 41.419 & 1.864 & Smail et al. (2004) \\
\hline SMMJ131222.35+423814.1 & 2.565 & 3.600 & 63.200 & 6.300 & Hainline (2008) \\
\hline SMMJ131222.35+423814.1 & 2.565 & 4.500 & 80.100 & 8.000 & Hainline (2008) \\
\hline SMMJ131222.35+423814.1 & 2.565 & 5.800 & 148.200 & 16.200 & Hainline (2008) \\
\hline SMMJ131222.35+423814.1 & 2.565 & 8.000 & 267.200 & 27.400 & Hainline (2008) \\
\hline SMMJ131222.35+423814.1 & 2.565 & 850.000 & 3000.000 & 900.000 & Chapman et al. (2005) \\
\hline SMMJ131222.35+423814.1 & 2.565 & 214000.000 & 26.300 & 3.900 & Chapman et al. (2005) \\
\hline SMMJ131225.20+424344.5 & 1.038 & 0.428 & 1.738 & 0.153 & Chapman et al. (2005) \\
\hline SMMJ131225.20+424344.5 & 1.038 & 0.630 & 1.787 & 0.157 & Fomalont et al. \\
\hline SMMJ131225.20+424344.5 & 1.038 & 0.656 & 5.248 & 0.462 & Chapman et al. (2005) \\
\hline SMMJ131225.20+424344.5 & 1.038 & 0.767 & 8.057 & 0.074 & Smail et al. (2004) \\
\hline SMMJ131225.20+424344.5 & 1.038 & 0.920 & 7.348 & 0.647 & Fomalont et al. (2006) \\
\hline SMMJ131225.20+424344.5 & 1.038 & 1.250 & 14.128 & 0.760 & Smail et al. (2004) \\
\hline SMMJ131225.20+424344.5 & 1.038 & 2.170 & 31.419 & 1.136 & Smail et al. (2004) \\
\hline SMMJ131225.20+424344.5 & 1.038 & 3.600 & 60.700 & 6.100 & Hainline (2008) \\
\hline SMMJ131225.20+424344.5 & 1.038 & 4.500 & 48.300 & 4.900 & Hainline (2008) \\
\hline SMMJ131225.20+424344.5 & 1.038 & 5.800 & 35.300 & 4.600 & Hainline (2008) \\
\hline SMMJ131225.20+424344.5 & 1.038 & 8.000 & 53.100 & 6.100 & Hainline (2008) \\
\hline SMMJ131225.20+424344.5 & 1.038 & 850.000 & 2400.000 & 800.000 & Chapman et al. (2005) \\
\hline SMMJ131225.20+424344.5 & 1.038 & 214000.000 & 76.400 & 6.800 & Chapman et al. (2005) \\
\hline SMMJ131225.73+423941.4 & 1.554 & 0.428 & 0.631 & 0.056 & Chapman et al. (2005) \\
\hline SMMJ131225.73+423941.4 & 1.554 & 0.630 & 0.179 & 0.016 & Fomalont et al. (2006) \\
\hline SMMJ131225.73+423941.4 & 1.554 & 0.656 & 0.759 & 0.067 & Chapman et al. (2005) \\
\hline SMMJ131225.73+423941.4 & 1.554 & 0.920 & 0.735 & 0.065 & Fomalont et al. (2006) \\
\hline SMMJ131225.73+423941.4 & 1.554 & 3.600 & 21.100 & 2.100 & Hainline (2008) \\
\hline SMMJ131225.73+423941.4 & 1.554 & 4.500 & 20.200 & 2.100 & Hainline (2008) \\
\hline SMMJ131225.73+423941.4 & 1.554 & 850.000 & 4100.000 & 1300.000 & Chapman et al. (2005) \\
\hline SMMJ131225.73+423941.4 & 1.554 & 214000.000 & 752.500 & 4.200 & Chapman et al. (2005) \\
\hline SMMJ131228.30+424454.8 & 2.931 & 0.428 & 0.158 & 0.014 & Chapman et al. (2005) \\
\hline SMMJ131228.30+424454.8 & 2.931 & 0.630 & 0.310 & 0.027 & Fomalont et al. (2006) \\
\hline SMMJ131228.30+424454.8 & 2.931 & 0.656 & 0.479 & 0.042 & Chapman et al. (2005) \\
\hline SMMJ131228.30+424454.8 & 2.931 & 0.767 & 0.721 & 0.070 & Smail et al. (2004) \\
\hline SMMJ131228.30+424454.8 & 2.931 & 0.920 & 0.611 & 0.054 & Fomalont et al. (2006) \\
\hline SMMJ131228.30+424454.8 & 2.931 & 1.250 & 2.302 & 0.523 & Smail et al. (2004) \\
\hline SMMJ131228.30+424454.8 & 2.931 & 3.600 & 11.300 & 1.200 & Hainline (2008) \\
\hline SMMJ131228.30+424454.8 & 2.931 & 4.500 & 13.900 & 1.400 & Hainline (2008) \\
\hline SMMJ131228.30+424454.8 & 2.931 & 5.800 & 21.700 & 3.300 & Hainline (2008) \\
\hline SMMJ131228.30+424454.8 & 2.931 & 8.000 & 26.500 & 3.500 & Hainline (2008) \\
\hline SMMJ131228.30+424454.8 & 2.931 & 850.000 & 3400.000 & 900.000 & Chapman et al. (2005) \\
\hline SMMJ131228.30+424454.8 & 2.931 & 214000.000 & 50.900 & 8.100 & Chapman et al. (2005) \\
\hline SMMJ131231.07+424609.0 & 2.713 & 0.656 & 0.063 & 0.006 & Chapman et al. (2005) \\
\hline SMMJ131231.07+424609.0 & 2.713 & 0.767 & 0.295 & 0.067 & Smail et al. (2004) \\
\hline SMMJ131231.07+424609.0 & 2.713 & 850.000 & 4900.000 & 1600.000 & Chapman et al. (2005) \\
\hline SMMJ131231.07+424609.0 & 2.713 & 214000.000 & 39.400 & 8.500 & Chapman et al. (2005) \\
\hline SMMJ131232.31+423949.5 & 2.320 & 0.428 & 0.191 & 0.017 & Chapman et al. (2005) \\
\hline SMMJ131232.31+423949.5 & 2.320 & 0.630 & 0.409 & 0.036 & Fomalont et al. (2006) \\
\hline SMMJ131232.31+423949.5 & 2.320 & 0.656 & 0.525 & 0.046 & Chapman et al. (2005) \\
\hline SMMJ131232.31+423949.5 & 2.320 & 0.767 & 0.987 & 0.078 & Smail et al. (2004) \\
\hline SMMJ131232.31+423949.5 & 2.320 & 0.920 & 0.557 & 0.049 & Fomalont et al. (2006) \\
\hline SMMJ131232.31+423949.5 & 2.320 & 1.250 & 3.178 & 0.607 & Smail et al. (2004) \\
\hline SMMJ131232.31+423949.5 & 2.320 & 2.170 & 9.401 & 0.984 & Smail et al. (2004) \\
\hline SMMJ131232.31+423949.5 & 2.320 & 3.600 & 26.900 & 2.700 & Hainline (2008) \\
\hline SMMJ131232.31+423949.5 & 2.320 & 4.500 & 42.600 & 4.300 & Hainline (2008) \\
\hline SMMJ131232.31+423949.5 & 2.320 & 5.800 & 76.300 & 8.900 & Hainline (2008) \\
\hline SMMJ131232.31+423949.5 & 2.320 & 8.000 & 191.400 & 19.800 & Hainline (2008) \\
\hline SMMJ131232.31+423949.5 & 2.320 & 850.000 & 4700.000 & 1100.000 & Chapman et al. (2005) \\
\hline
\end{tabular}


Table A.1. continued

\begin{tabular}{|c|c|c|c|c|c|}
\hline SMG & $z$ & $\begin{array}{r}\lambda_{\mathrm{obs}} \\
(\mu \mathrm{m})\end{array}$ & $\begin{array}{c}\text { Flux } \\
(\mu \mathrm{Jy})\end{array}$ & $\begin{array}{l}\text { Error } \\
(\mu \mathrm{Jy})\end{array}$ & Reference \\
\hline SMMJ131232.31+423949.5 & 2.320 & 214000.000 & 94.800 & 4.300 & Chapman et al. (2005) \\
\hline SMMJ131239.14+424155.7 & 2.242 & 0.428 & 0.145 & 0.013 & Chapman et al. (2005) \\
\hline SMMJ131239.14+424155.7 & 2.242 & 0.630 & 0.163 & 0.014 & Fomalont et al. (2006) \\
\hline SMMJ131239.14+424155.7 & 2.242 & 0.656 & 0.191 & 0.017 & Chapman et al. (2005) \\
\hline SMMJ131239.14+424155.7 & 2.242 & 0.767 & 0.784 & 0.062 & Smail et al. (2004) \\
\hline SMMJ131239.14+424155.7 & 2.242 & 0.920 & 0.352 & 0.031 & Fomalont et al. (2006) \\
\hline SMMJ131239.14+424155.7 & 2.242 & 2.170 & 10.500 & 1.522 & Smail et al. (2004) \\
\hline SMMJ131239.14+424155.7 & 2.242 & 3.600 & 9.300 & 1.000 & Hainline (2008) \\
\hline SMMJ131239.14+424155.7 & 2.242 & 4.500 & 10.300 & 1.100 & Hainline (2008) \\
\hline SMMJ131239.14+424155.7 & 2.242 & 8.000 & 15.900 & 3.600 & Hainline (2008) \\
\hline SMMJ131239.14+424155.7 & 2.242 & 850.000 & 7400.000 & 1900.000 & Chapman et al. (2005) \\
\hline SMMJ131239.14+424155.7 & 2.242 & 214000.000 & 49.800 & 6.600 & Chapman et al. (2005) \\
\hline SMMJ141741.81+522823.0 & 1.150 & 0.428 & 2.291 & 0.202 & Chapman et al. (2005) \\
\hline SMMJ141741.81+522823.0 & 1.150 & 0.656 & 9.120 & 0.802 & Chapman et al. (2005) \\
\hline SMMJ141741.81+522823.0 & 1.150 & 0.767 & 19.328 & 4.666 & Smail et al. (2004) \\
\hline SMMJ141741.81+522823.0 & 1.150 & 1.250 & 59.441 & 1.085 & Smail et al. (2004) \\
\hline SMMJ141741.81+522823.0 & 1.150 & 2.170 & 172.661 & 15.192 & Smail et al. (2004) \\
\hline SMMJ141741.81+522823.0 & 1.150 & 3.600 & 518.700 & 51.900 & Hainline (2008) \\
\hline SMMJ141741.81+522823.0 & 1.150 & 4.500 & 851.500 & 85.200 & Hainline (2008) \\
\hline SMMJ141741.81+522823.0 & 1.150 & 5.800 & 1313.400 & 134.200 & Hainline (2008) \\
\hline SMMJ141741.81+522823.0 & 1.150 & 8.000 & 1961.900 & 197.100 & Hainline (2008) \\
\hline SMMJ141741.81+522823.0 & 1.150 & 24.000 & 5741.600 & 288.500 & Hainline (2008) \\
\hline SMMJ141741.81+522823.0 & 1.150 & 70.000 & 18700.000 & 3500.000 & Hainline (2008) \\
\hline SMMJ141741.81+522823.0 & 1.150 & 850.000 & 3300.000 & 1000.000 & Chapman et al. (2005) \\
\hline SMMJ141741.81+522823.0 & 1.150 & 214000.000 & 80.000 & 16.000 & Chapman et al. (2005) \\
\hline SMMJ141742.04+523025.7 & 0.661 & 0.428 & 47.863 & 4.211 & Chapman et al. (2005) \\
\hline SMMJ141742.04+523025.7 & 0.661 & 0.656 & 63.096 & 5.552 & Chapman et al. (2005) \\
\hline SMMJ141742.04+523025.7 & 0.661 & 0.767 & 16.076 & 2.705 & Smail et al. (2004) \\
\hline SMMJ141742.04+523025.7 & 0.661 & 1.250 & 46.783 & 0.854 & Smail et al. (2004) \\
\hline SMMJ141742.04+523025.7 & 0.661 & 2.170 & 143.614 & 12.636 & Smail et al. (2004) \\
\hline SMMJ141742.04+523025.7 & 0.661 & 3.600 & 153.200 & 15.300 & Hainline (2008) \\
\hline SMMJ141742.04+523025.7 & 0.661 & 4.500 & 126.100 & 12.600 & Hainline (2008) \\
\hline SMMJ141742.04+523025.7 & 0.661 & 5.800 & 140.400 & 14.100 & Hainline (2008) \\
\hline SMMJ141742.04+523025.7 & 0.661 & 8.000 & 152.600 & 15.300 & Hainline (2008) \\
\hline SMMJ141742.04+523025.7 & 0.661 & 24.000 & 1090.100 & 56.800 & Hainline (2008) \\
\hline SMMJ141742.04+523025.7 & 0.661 & 70.000 & 16900.000 & 4000.000 & Hainline (2008) \\
\hline SMMJ141742.04+523025.7 & 0.661 & 850.000 & 2600.000 & 900.000 & Chapman et al. (2005) \\
\hline SMMJ141742.04+523025.7 & 0.661 & 214000.000 & 232.000 & 23.000 & Chapman et al. (2005) \\
\hline SMMJ141750.50+523101.0 & 2.128 & 0.428 & 0.331 & 0.029 & Chapman et al. (2005) \\
\hline SMMJ141750.50+523101.0 & 2.128 & 0.656 & 0.363 & 0.032 & Chapman et al. (2005) \\
\hline SMMJ141750.50+523101.0 & 2.128 & 2.170 & 2.076 & 0.183 & Smail et al. (2004) \\
\hline SMMJ141750.50+523101.0 & 2.128 & 850.000 & 2800.000 & 900.000 & Chapman et al. (2005) \\
\hline SMMJ141750.50+523101.0 & 2.128 & 214000.000 & 57.000 & 14.000 & Chapman et al. (2005) \\
\hline SMMJ141800.40+512820.3 & 1.913 & 0.428 & 0.832 & 0.073 & Chapman et al. (2005) \\
\hline SMMJ141800.40+512820.3 & 1.913 & 0.656 & 1.738 & 0.153 & Chapman et al. (2005) \\
\hline SMMJ141800.40+512820.3 & 1.913 & 850.000 & 5000.000 & 1000.000 & Chapman et al. (2005) \\
\hline SMMJ141800.40+512820.3 & 1.913 & 214000.000 & 128.000 & 19.000 & Chapman et al. (2005) \\
\hline SMMJ141802.87+523011.1 & 2.127 & 0.428 & 0.631 & 0.056 & Chapman et al. (2005) \\
\hline SMMJ141802.87+523011.1 & 2.127 & 0.656 & 0.759 & 0.067 & Chapman et al. (2005) \\
\hline SMMJ141802.87+523011.1 & 2.127 & 1.250 & 2.524 & 0.662 & Smail et al. (2004) \\
\hline SMMJ141802.87+523011.1 & 2.127 & 2.170 & 4.755 & 0.418 & Smail et al. (2004) \\
\hline SMMJ141802.87+523011.1 & 2.127 & 3.600 & 5.000 & 0.500 & Hainline (2008) \\
\hline SMMJ141802.87+523011.1 & 2.127 & 4.500 & 5.400 & 0.600 & Hainline (2008) \\
\hline SMMJ141802.87+523011.1 & 2.127 & 5.800 & 5.700 & 0.700 & Hainline (2008) \\
\hline SMMJ141802.87+523011.1 & 2.127 & 850.000 & 3400.000 & 900.000 & Chapman et al. (2005) \\
\hline SMMJ141802.87+523011.1 & 2.127 & 214000.000 & 39.000 & 14.000 & Chapman et al. (2005) \\
\hline SMMJ141809.00+522803.8 & 2.712 & 0.428 & 0.174 & 0.015 & Chapman et al. (2005) \\
\hline SMMJ141809.00+522803.8 & 2.712 & 0.656 & 0.191 & 0.017 & Chapman et al. (2005) \\
\hline SMMJ141809.00+522803.8 & 2.712 & 1.250 & 2.925 & 0.686 & Smail et al. (2004) \\
\hline SMMJ141809.00+522803.8 & 2.712 & 2.170 & 15.747 & 1.386 & Smail et al. (2004) \\
\hline SMMJ141809.00+522803.8 & 2.712 & 3.600 & 43.700 & 4.400 & Hainline (2008) \\
\hline SMMJ141809.00+522803.8 & 2.712 & 4.500 & 56.700 & 5.700 & Hainline (2008) \\
\hline
\end{tabular}


Table A.1. continued

\begin{tabular}{|c|c|c|c|c|c|}
\hline SMG & $z$ & $\begin{array}{r}\lambda_{\mathrm{obs}} \\
(\mu \mathrm{m})\end{array}$ & $\begin{array}{r}\text { Flux } \\
(\mu \mathrm{Jy})\end{array}$ & $\begin{array}{l}\text { Error } \\
(\mu \mathrm{Jy})\end{array}$ & Reference \\
\hline SMMJ141809.00+522803.8 & 2.712 & 5.800 & 60.800 & 6.400 & Hainline (2008) \\
\hline SMMJ141809.00+522803.8 & 2.712 & 8.000 & 40.800 & 5.300 & Hainline (2008) \\
\hline SMMJ141809.00+522803.8 & 2.712 & 850.000 & 4300.000 & 1000.000 & Chapman et al. (2005) \\
\hline SMMJ141809.00+522803.8 & 2.712 & 214000.000 & 67.000 & 15.000 & Chapman et al. (2005) \\
\hline SMMJ141813.54+522923.4 & 3.484 & 0.428 & 0.040 & 0.004 & Chapman et al. (2005) \\
\hline SMMJ141813.54+522923.4 & 3.484 & 0.656 & 0.158 & 0.014 & Chapman et al. (2005) \\
\hline SMMJ141813.54+522923.4 & 3.484 & 0.767 & 0.222 & 0.054 & Smail et al. (2004) \\
\hline SMMJ141813.54+522923.4 & 3.484 & 2.170 & 1.982 & 0.174 & Smail et al. (2004) \\
\hline SMMJ141813.54+522923.4 & 3.484 & 850.000 & 3600.000 & 1100.000 & Chapman et al. (2005) \\
\hline SMMJ141813.54+522923.4 & 3.484 & 214000.000 & 93.000 & 16.000 & Chapman et al. (2005) \\
\hline SMMJ163627.94+405811.2 & 3.180 & 0.428 & 0.302 & 0.027 & Chapman et al. (2005) \\
\hline SMMJ163627.94+405811.2 & 3.180 & 0.656 & 0.398 & 0.035 & Chapman et al. (2005) \\
\hline SMMJ163627.94+405811.2 & 3.180 & 0.767 & 0.996 & 0.071 & Smail et al. (2004) \\
\hline SMMJ163627.94+405811.2 & 3.180 & 3.600 & 3.700 & 0.400 & Hainline (2008) \\
\hline SMMJ163627.94+405811.2 & 3.180 & 4.500 & 5.100 & 0.600 & Hainline (2008) \\
\hline SMMJ163627.94+405811.2 & 3.180 & 850.000 & 6500.000 & 2100.000 & Chapman et al. (2005) \\
\hline SMMJ163627.94+405811.2 & 3.180 & 214000.000 & 92.000 & 23.000 & Chapman et al. (2005) \\
\hline SMMJ163631.47+405546.9 & 2.283 & 0.428 & 0.398 & 0.035 & Chapman et al. (2005) \\
\hline SMMJ163631.47+405546.9 & 2.283 & 0.656 & 0.832 & 0.073 & Chapman et al. (2005) \\
\hline SMMJ163631.47+405546.9 & 2.283 & 2.170 & 2.613 & 1.164 & Ivison et al. (2002) \\
\hline SMMJ163631.47+405546.9 & 2.283 & 3.600 & 9.500 & 1.000 & Hainline (2008) \\
\hline SMMJ163631.47+405546.9 & 2.283 & 4.500 & 17.000 & 1.800 & Hainline (2008) \\
\hline SMMJ163631.47+405546.9 & 2.283 & 5.800 & 23.500 & 3.000 & Hainline (2008) \\
\hline SMMJ163631.47+405546.9 & 2.283 & 8.000 & 19.500 & 2.600 & Hainline (2008) \\
\hline SMMJ163631.47+405546.9 & 2.283 & 24.000 & 240.200 & 41.500 & Hainline (2008) \\
\hline SMMJ163631.47+405546.9 & 2.283 & 350.000 & 38300.000 & 5500.000 & Kovács et al. (2006) \\
\hline SMMJ163631.47+405546.9 & 2.283 & 850.000 & 6300.000 & 1900.000 & Chapman et al. (2005) \\
\hline SMMJ163631.47+405546.9 & 2.283 & 214000.000 & 99.000 & 23.000 & Chapman et al. (2005) \\
\hline SMMJ163639.01+405635.9 & 1.495 & 0.428 & 0.525 & 0.046 & Chapman et al. (2005); Menéndez-Delmestre et al. (2009) \\
\hline SMMJ163639.01+405635.9 & 1.495 & 0.550 & 1.245 & 0.067 & Ivison et al. (2002) \\
\hline SMMJ163639.01+405635.9 & 1.495 & 0.656 & 1.282 & 0.035 & Ivison et al. (2002) \\
\hline SMMJ163639.01+405635.9 & 1.495 & 0.656 & 1.096 & 0.096 & Chapman et al. (2005) \\
\hline SMMJ163639.01+405635.9 & 1.495 & 0.767 & 2.571 & 0.070 & Ivison et al. (2002) \\
\hline SMMJ163639.01+405635.9 & 1.495 & 2.170 & 10.028 & 0.539 & Ivison et al. (2002) \\
\hline SMMJ163639.01+405635.9 & 1.495 & 3.600 & 23.000 & 2.300 & Hainline (2008) \\
\hline SMMJ163639.01+405635.9 & 1.495 & 4.500 & 29.300 & 2.900 & Hainline (2008) \\
\hline SMMJ163639.01+405635.9 & 1.495 & 5.800 & 33.500 & 3.600 & Hainline (2008) \\
\hline SMMJ163639.01+405635.9 & 1.495 & 8.000 & 40.000 & 4.200 & Hainline (2008) \\
\hline SMMJ163639.01+405635.9 & 1.495 & 24.000 & 321.400 & 38.600 & Hainline (2008) \\
\hline SMMJ163639.01+405635.9 & 1.495 & 850.000 & 5100.000 & 1400.000 & Chapman et al. (2005) \\
\hline SMMJ163639.01+405635.9 & 1.495 & 1200.000 & 3400.000 & 700.000 & Greve et al. (2004) \\
\hline SMMJ163639.01+405635.9 & 1.495 & 214000.000 & 159.000 & 27.000 & Chapman et al. (2005) \\
\hline SMMJ163650.43+405734.5 & 2.378 & 0.428 & 1.738 & 0.153 & Chapman et al. (2005); Menéndez-Delmestre et al. (2009) \\
\hline SMMJ163650.43+405734.5 & 2.378 & 0.550 & 4.047 & 0.110 & Ivison et al. (2002) \\
\hline SMMJ163650.43+405734.5 & 2.378 & 0.656 & 3.802 & 0.035 & Ivison et al. (2002) \\
\hline SMMJ163650.43+405734.5 & 2.378 & 0.656 & 3.631 & 0.319 & Chapman et al. (2005) \\
\hline SMMJ163650.43+405734.5 & 2.378 & 0.767 & 4.510 & 0.082 & Ivison et al. (2002) \\
\hline SMMJ163650.43+405734.5 & 2.378 & 2.170 & 27.874 & 0.509 & Ivison et al. (2002) \\
\hline SMMJ163650.43+405734.5 & 2.378 & 3.600 & 31.000 & 3.100 & Hainline (2008) \\
\hline SMMJ163650.43+405734.5 & 2.378 & 4.500 & 36.900 & 3.700 & Hainline (2008) \\
\hline SMMJ163650.43+405734.5 & 2.378 & 5.800 & 50.500 & 5.200 & Hainline (2008) \\
\hline SMMJ163650.43+405734.5 & 2.378 & 8.000 & 65.000 & 6.600 & Hainline (2008) \\
\hline SMMJ163650.43+405734.5 & 2.378 & 24.000 & 886.500 & 58.800 & Hainline (2008) \\
\hline SMMJ163650.43+405734.5 & 2.378 & 350.000 & 33000.000 & 5600.000 & Kovács et al. (2006) \\
\hline SMMJ163650.43+405734.5 & 2.378 & 850.000 & 8200.000 & 1700.000 & Chapman et al. (2005) \\
\hline SMMJ163650.43+405734.5 & 2.378 & 1200.000 & 3100.000 & 700.000 & Greve et al. (2004) \\
\hline SMMJ163650.43+405734.5 & 2.378 & 1300.000 & 2600.000 & 500.000 & Tacconi et al. (2006) \\
\hline SMMJ163650.43+405734.5 & 2.378 & 214000.000 & 221.000 & 16.000 & Chapman et al. (2005) \\
\hline SMMJ163658.19+410523.8 & 2.454 & 0.428 & 0.145 & 0.013 & Chapman et al. (2005) \\
\hline SMMJ163658.19+410523.8 & 2.454 & 0.656 & 0.211 & 0.069 & Ivison et al. (2002) \\
\hline SMMJ163658.19+410523.8 & 2.454 & 0.656 & 0.174 & 0.015 & Chapman et al. (2005) \\
\hline SMMJ163658.19+410523.8 & 2.454 & 0.767 & 0.393 & 0.066 & Ivison et al. (2002) \\
\hline SMMJ163658.19+410523.8 & 2.454 & 2.170 & 8.113 & 0.436 & Ivison et al. (2002) \\
\hline SMMJ163658.19+410523.8 & 2.454 & 3.600 & 14.900 & 1.500 & Hainline (2008) \\
\hline
\end{tabular}


Table A.1. continued

\begin{tabular}{|c|c|c|c|c|c|}
\hline SMG & $z$ & $\begin{array}{r}\lambda_{\mathrm{obs}} \\
(\mu \mathrm{m})\end{array}$ & $\begin{array}{l}\text { Flux } \\
(\mu \mathrm{Jy})\end{array}$ & $\begin{array}{l}\text { Error } \\
(\mu \mathrm{Jy})\end{array}$ & Reference \\
\hline SMMJ163658.19+410523.8 & 2.454 & 4.500 & 21.200 & 2.100 & Hainline (2008) \\
\hline SMMJ163658.19+410523.8 & 2.454 & 5.800 & 28.100 & 3.100 & Hainline (2008) \\
\hline SMMJ163658.19+410523.8 & 2.454 & 8.000 & 29.000 & 3.500 & Hainline (2008) \\
\hline SMMJ163658.19+410523.8 & 2.454 & 24.000 & 343.500 & 46.800 & Hainline (2008) \\
\hline SMMJ163658.19+410523.8 & 2.454 & 350.000 & 45200.000 & 5300.000 & Kovács et al. (2006) \\
\hline SMMJ163658.19+410523.8 & 2.454 & 850.000 & 10700.000 & 2000.000 & Chapman et al. (2005) \\
\hline SMMJ163658.19+410523.8 & 2.454 & 1200.000 & 3400.000 & 1100.000 & Greve et al. (2004) \\
\hline SMMJ163658.19+410523.8 & 2.454 & 1300.000 & 1500.000 & 500.000 & Tacconi et al. (2006) \\
\hline SMMJ163658.19+410523.8 & 2.454 & 214000.000 & 92.000 & 16.000 & Chapman et al. (2005) \\
\hline SMMJ163658.78+405728.1 & 1.190 & 0.428 & 1.000 & 0.088 & Chapman et al. (2005); Valiante et al. (2007) \\
\hline SMMJ163658.78+405728.1 & 1.190 & 0.550 & 2.825 & 0.077 & Ivison et al. (2002) \\
\hline SMMJ163658.78+405728.1 & 1.190 & 0.656 & 3.133 & 0.057 & Ivison et al. (2002) \\
\hline SMMJ163658.78+405728.1 & 1.190 & 0.656 & 3.020 & 0.266 & Chapman et al. (2005) \\
\hline SMMJ163658.78+405728.1 & 1.190 & 0.767 & 5.837 & 0.107 & Ivison et al. (2002) \\
\hline SMMJ163658.78+405728.1 & 1.190 & 2.170 & 36.074 & 2.870 & Ivison et al. (2002) \\
\hline SMMJ163658.78+405728.1 & 1.190 & 3.600 & 67.500 & 6.800 & Hainline (2008) \\
\hline SMMJ163658.78+405728.1 & 1.190 & 4.500 & 66.600 & 6.700 & Hainline (2008) \\
\hline SMMJ163658.78+405728.1 & 1.190 & 5.800 & 48.800 & 5.200 & Hainline (2008) \\
\hline SMMJ163658.78+405728.1 & 1.190 & 8.000 & 61.700 & 6.300 & Hainline (2008) \\
\hline SMMJ163658.78+405728.1 & 1.190 & 24.000 & 463.000 & 42.000 & Hainline (2008) \\
\hline SMMJ163658.78+405728.1 & 1.190 & 850.000 & 5100.000 & 1400.000 & Chapman et al. (2005) \\
\hline SMMJ163658.78+405728.1 & 1.190 & 214000.000 & 74.000 & 29.000 & Chapman et al. (2005) \\
\hline SMMJ163704.34+410530.3 & 0.840 & 0.428 & 0.759 & 0.067 & Chapman et al. (2005) \\
\hline SMMJ163704.34+410530.3 & 0.840 & 0.550 & 1.955 & 0.053 & Ivison et al. (2002) \\
\hline SMMJ163704.34+410530.3 & 0.840 & 0.656 & 2.089 & 0.038 & Ivison et al. (2002) \\
\hline SMMJ163704.34+410530.3 & 0.840 & 0.656 & 2.089 & 0.184 & Chapman et al. (2005) \\
\hline SMMJ163704.34+410530.3 & 0.840 & 0.767 & 3.892 & 0.106 & Ivison et al. (2002) \\
\hline SMMJ163704.34+410530.3 & 0.840 & 2.170 & 10.597 & 2.102 & Ivison et al. (2002) \\
\hline SMMJ163704.34+410530.3 & 0.840 & 4.500 & 12.500 & 1.300 & Hainline (2008) \\
\hline SMMJ163704.34+410530.3 & 0.840 & 350.000 & 21000.000 & 4700.000 & Kovács et al. (2006) \\
\hline SMMJ163704.34+410530.3 & 0.840 & 850.000 & 11200.000 & 1600.000 & Chapman et al. (2005) \\
\hline SMMJ163704.34+410530.3 & 0.840 & 214000.000 & 45.000 & 16.000 & Chapman et al. (2005) \\
\hline SMMJ163706.51+405313.8 & 2.374 & 0.656 & 0.525 & 0.046 & Chapman et al. (2005); Valiante et al. (2007) \\
\hline SMMJ163706.51+405313.8 & 2.374 & 0.767 & 1.301 & 0.147 & Smail et al. (2004) \\
\hline SMMJ163706.51+405313.8 & 2.374 & 2.170 & 14.230 & 0.765 & Smail et al. (2004) \\
\hline SMMJ163706.51+405313.8 & 2.374 & 4.500 & 31.500 & 3.200 & Hainline (2008) \\
\hline SMMJ163706.51+405313.8 & 2.374 & 8.000 & 62.000 & 6.300 & Hainline (2008) \\
\hline SMMJ163706.51+405313.8 & 2.374 & 24.000 & 390.300 & 50.700 & Hainline (2008) \\
\hline SMMJ163706.51+405313.8 & 2.374 & 350.000 & 36100.000 & 7700.000 & Kovács et al. (2006) \\
\hline SMMJ163706.51+405313.8 & 2.374 & 850.000 & 11200.000 & 2900.000 & Chapman et al. (2005) \\
\hline SMMJ163706.51+405313.8 & 2.374 & 1200.000 & 4200.000 & 1100.000 & Greve et al. (2004) \\
\hline SMMJ163706.51+405313.8 & 2.374 & 214000.000 & 74.000 & 23.000 & Chapman et al. (2005) \\
\hline SMMJ221724.69+001242.1 & 0.510 & 0.428 & 25.119 & 2.210 & Chapman et al. (2005) \\
\hline SMMJ221724.69+001242.1 & 0.510 & 0.656 & 36.308 & 3.195 & Chapman et al. (2005) \\
\hline SMMJ221724.69+001242.1 & 0.510 & 3.600 & 188.000 & 18.800 & Hainline (2008) \\
\hline SMMJ221724.69+001242.1 & 0.510 & 4.500 & 146.400 & 14.600 & Hainline (2008) \\
\hline SMMJ221724.69+001242.1 & 0.510 & 5.800 & 131.800 & 13.400 & Hainline (2008) \\
\hline SMMJ221724.69+001242.1 & 0.510 & 8.000 & 128.000 & 12.900 & Hainline (2008) \\
\hline SMMJ221724.69+001242.1 & 0.510 & 24.000 & 618.100 & 45.000 & Hainline (2008) \\
\hline SMMJ221724.69+001242.1 & 0.510 & 850.000 & 8600.001 & 1900.000 & Chapman et al. (2005) \\
\hline SMMJ221724.69+001242.1 & 0.510 & 214000.000 & 121.100 & 10.700 & Chapman et al. (2005) \\
\hline SMMJ221725.97+001238.9 & 3.094 & 0.573 & 0.068 & 0.006 & Chapman et al. (2003b) \\
\hline SMMJ221725.97+001238.9 & 3.094 & 0.656 & 0.052 & 0.005 & Chapman et al. (2005) \\
\hline SMMJ221725.97+001238.9 & 3.094 & 0.767 & 0.088 & 0.008 & Chapman et al. (2003b) \\
\hline SMMJ221725.97+001238.9 & 3.094 & 0.767 & 1.165 & 0.073 & Smail et al. (2004) \\
\hline SMMJ221725.97+001238.9 & 3.094 & 3.600 & 5.900 & 0.600 & Hainline (2008) \\
\hline SMMJ221725.97+001238.9 & 3.094 & 4.500 & 7.600 & 0.800 & Hainline (2008) \\
\hline SMMJ221725.97+001238.9 & 3.094 & 5.800 & 9.000 & 1.200 & Hainline (2008) \\
\hline SMMJ221725.97+001238.9 & 3.094 & 8.000 & 14.600 & 1.700 & Hainline (2008) \\
\hline SMMJ221725.97+001238.9 & 3.094 & 850.000 & 17400.000 & 2900.000 & Chapman et al. (2005) \\
\hline SMMJ221725.97+001238.9 & 3.094 & 214000.000 & 41.200 & 9.300 & Chapman et al. (2005) \\
\hline SMMJ221733.02+000906.0 & 0.926 & 0.428 & 0.575 & 0.051 & Chapman et al. (2005); Menéndez-Delmestre et al. (2009) \\
\hline
\end{tabular}


Table A.1. continued

\begin{tabular}{|c|c|c|c|c|c|}
\hline SMG & $z$ & $\begin{array}{r}\lambda_{\mathrm{obs}} \\
(\mu \mathrm{m})\end{array}$ & $\begin{array}{c}\text { Flux } \\
(\mu \mathrm{Jy})\end{array}$ & $\begin{array}{l}\text { Error } \\
(\mu \mathrm{Jy})\end{array}$ & Reference \\
\hline SMMJ221733.02+000906.0 & 0.926 & 0.656 & 1.096 & 0.096 & Chapman et al. (2005) \\
\hline SMMJ221733.02+000906.0 & 0.926 & 0.767 & 0.267 & 0.023 & Smail et al. (2004) \\
\hline SMMJ221733.02+000906.0 & 0.926 & 1.250 & 20.801 & 0.936 & Smail et al. (2004) \\
\hline SMMJ221733.02+000906.0 & 0.926 & 2.170 & 54.600 & 1.488 & Smail et al. (2004) \\
\hline SMMJ221733.02+000906.0 & 0.926 & 850.000 & 11100.000 & 3400.000 & Chapman et al. (2005) \\
\hline SMMJ221733.02+000906.0 & 0.926 & 214000.000 & 161.700 & 16.300 & Chapman et al. (2005) \\
\hline SMMJ221733.12+001120.2 & 0.652 & 0.428 & 2.754 & 0.242 & Chapman et al. (2005); Menéndez-Delmestre et al. (2009) \\
\hline SMMJ221733.12+001120.2 & 0.652 & 0.656 & 10.000 & 0.880 & Chapman et al. (2005) \\
\hline SMMJ221733.12+001120.2 & 0.652 & 0.767 & 19.687 & 0.180 & Smail et al. (2004) \\
\hline SMMJ221733.12+001120.2 & 0.652 & 1.250 & 35.163 & 0.642 & Smail et al. (2004) \\
\hline SMMJ221733.12+001120.2 & 0.652 & 2.170 & 113.030 & 2.063 & Smail et al. (2004) \\
\hline SMMJ221733.12+001120.2 & 0.652 & 3.600 & 87.900 & 8.800 & Hainline (2008) \\
\hline SMMJ221733.12+001120.2 & 0.652 & 4.500 & 66.500 & 6.700 & Hainline (2008) \\
\hline SMMJ221733.12+001120.2 & 0.652 & 5.800 & 78.800 & 8.100 & Hainline (2008) \\
\hline SMMJ221733.12+001120.2 & 0.652 & 8.000 & 66.100 & 6.700 & Hainline (2008) \\
\hline SMMJ221733.12+001120.2 & 0.652 & 24.000 & 516.900 & 45.600 & Hainline (2008) \\
\hline SMMJ221733.12+001120.2 & 0.652 & 850.000 & 6900.000 & 2100.000 & Chapman et al. (2005) \\
\hline SMMJ221733.12+001120.2 & 0.652 & 214000.000 & 69.200 & 10.300 & Chapman et al. (2005) \\
\hline SMMJ221733.91+001352.1 & 2.555 & 0.428 & 0.437 & 0.038 & Chapman et al. (2005); Menéndez-Delmestre et al. (2009) \\
\hline SMMJ221733.91+001352.1 & 2.555 & 0.656 & 0.832 & 0.073 & Chapman et al. (2005) \\
\hline SMMJ221733.91+001352.1 & 2.555 & 0.767 & 0.951 & 0.068 & Smail et al. (2004) \\
\hline SMMJ221733.91+001352.1 & 2.555 & 1.250 & 2.643 & 0.544 & Smail et al. (2004) \\
\hline SMMJ221733.91+001352.1 & 2.555 & 3.600 & 21.100 & 2.100 & Hainline (2008) \\
\hline SMMJ221733.91+001352.1 & 2.555 & 4.500 & 26.600 & 2.700 & Hainline (2008) \\
\hline SMMJ221733.91+001352.1 & 2.555 & 5.800 & 28.600 & 3.000 & Hainline (2008) \\
\hline SMMJ221733.91+001352.1 & 2.555 & 8.000 & 38.900 & 4.000 & Hainline (2008) \\
\hline SMMJ221733.91+001352.1 & 2.555 & 850.000 & 9100.000 & 1100.000 & Chapman et al. (2005) \\
\hline SMMJ221733.91+001352.1 & 2.555 & 214000.000 & 44.500 & 13.400 & Chapman et al. (2005) \\
\hline SMMJ221735.15+001537.2 & 3.098 & 0.428 & 0.132 & 0.012 & Chapman et al. (2005); Menéndez-Delmestre et al. (2009) \\
\hline SMMJ221735.15+001537.2 & 3.098 & 0.656 & 0.191 & 0.017 & Chapman et al. (2005) \\
\hline SMMJ221735.15+001537.2 & 3.098 & 0.767 & 0.900 & 0.064 & Smail et al. (2004) \\
\hline SMMJ221735.15+001537.2 & 3.098 & 1.250 & 2.410 & 0.368 & Smail et al. (2004) \\
\hline SMMJ221735.15+001537.2 & 3.098 & 2.170 & 5.072 & 0.614 & Smail et al. (2004) \\
\hline SMMJ221735.15+001537.2 & 3.098 & 3.600 & 4.200 & 0.400 & Hainline (2008) \\
\hline SMMJ221735.15+001537.2 & 3.098 & 4.500 & 6.100 & 0.600 & Hainline (2008) \\
\hline SMMJ221735.15+001537.2 & 3.098 & 8.000 & 8.600 & 1.300 & Hainline (2008) \\
\hline SMMJ221735.15+001537.2 & 3.098 & 850.000 & 6300.000 & 1300.000 & Chapman et al. (2005) \\
\hline SMMJ221735.15+001537.2 & 3.098 & 214000.000 & 49.400 & 13.300 & Chapman et al. (2005) \\
\hline SMMJ221735.84+001558.9 & 3.089 & 0.428 & 0.083 & 0.007 & Chapman et al. (2005); Menéndez-Delmestre et al. (2009) \\
\hline SMMJ221735.84+001558.9 & 3.089 & 0.656 & 0.229 & 0.020 & Chapman et al. (2005) \\
\hline SMMJ221735.84+001558.9 & 3.089 & 0.767 & 0.362 & 0.061 & Smail et al. (2004) \\
\hline SMMJ221735.84+001558.9 & 3.089 & 1.250 & 1.265 & 0.305 & Smail et al. (2004) \\
\hline SMMJ221735.84+001558.9 & 3.089 & 2.170 & 2.662 & 0.528 & Smail et al. (2004) \\
\hline SMMJ221735.84+001558.9 & 3.089 & 3.600 & 6.900 & 0.700 & Hainline (2008) \\
\hline SMMJ221735.84+001558.9 & 3.089 & 4.500 & 11.100 & 1.100 & Hainline (2008) \\
\hline SMMJ221735.84+001558.9 & 3.089 & 5.800 & 12.600 & 1.500 & Hainline (2008) \\
\hline SMMJ221735.84+001558.9 & 3.089 & 8.000 & 17.700 & 2.000 & Hainline (2008) \\
\hline SMMJ221735.84+001558.9 & 3.089 & 850.000 & 4900.000 & 1300.000 & Chapman et al. (2005) \\
\hline SMMJ221735.84+001558.9 & 3.089 & 214000.000 & 44.300 & 12.800 & Chapman et al. (2005) \\
\hline SMMJ221737.39+001025.1 & 2.614 & 0.428 & 0.331 & 0.029 & Chapman et al. (2005); Menéndez-Delmestre et al. (2009) \\
\hline SMMJ221737.39+001025.1 & 2.614 & 0.656 & 0.525 & 0.046 & Chapman et al. (2005) \\
\hline SMMJ221737.39+001025.1 & 2.614 & 0.767 & 0.813 & 0.065 & Smail et al. (2004) \\
\hline SMMJ221737.39+001025.1 & 2.614 & 1.250 & 2.219 & 0.390 & Smail et al. (2004) \\
\hline SMMJ221737.39+001025.1 & 2.614 & 2.170 & 9.401 & 0.906 & Smail et al. (2004) \\
\hline SMMJ221737.39+001025.1 & 2.614 & 850.000 & 6100.000 & 2000.000 & Chapman et al. (2005) \\
\hline SMMJ221737.39+001025.1 & 2.614 & 214000.000 & 110.100 & 14.000 & Chapman et al. (2005) \\
\hline SMMJ221804.42+002154.4 & 2.517 & 0.428 & 0.302 & 0.027 & Chapman et al. (2005); Menéndez-Delmestre et al. (2009) \\
\hline SMMJ221804.42+002154.4 & 2.517 & 0.656 & 0.479 & 0.042 & Chapman et al. (2005) \\
\hline SMMJ221804.42+002154.4 & 2.517 & 0.767 & 1.439 & 0.065 & Smail et al. (2004) \\
\hline SMMJ221804.42+002154.4 & 2.517 & 2.170 & 4.418 & 0.941 & Smail et al. (2004) \\
\hline SMMJ221804.42+002154.4 & 2.517 & 3.600 & 9.100 & 0.900 & Hainline (2008) \\
\hline SMMJ221804.42+002154.4 & 2.517 & 4.500 & 13.400 & 1.400 & Hainline (2008) \\
\hline SMMJ221804.42+002154.4 & 2.517 & 5.800 & 19.500 & 2.300 & Hainline (2008) \\
\hline SMMJ221804.42+002154.4 & 2.517 & 24.000 & 346.700 & 38.800 & Hainline (2008) \\
\hline SMMJ221804.42+002154.4 & 2.517 & 850.000 & 9000.001 & 2300.000 & Chapman et al. (2005) \\
\hline
\end{tabular}


Table A.1. continued

\begin{tabular}{|c|c|c|c|c|c|}
\hline SMG & $z$ & $\begin{array}{r}\lambda_{\mathrm{obs}} \\
(\mu \mathrm{m})\end{array}$ & $\begin{array}{r}\text { Flux } \\
(\mu \mathrm{Jy})\end{array}$ & $\begin{array}{l}\text { Error } \\
(\mu \mathrm{Jy})\end{array}$ & Reference \\
\hline SMMJ221804.42+002154.4 & 2.517 & 214000.000 & 43.800 & 10.400 & Chapman et al. (2005) \\
\hline SMMJ221806.77+001245.7 & 3.623 & 0.428 & 0.174 & 0.015 & Chapman et al. (2005); Menéndez-Delmestre et al. (2009) \\
\hline SMMJ221806.77+001245.7 & 3.623 & 0.656 & 0.692 & 0.061 & Chapman et al. (2005) \\
\hline SMMJ221806.77+001245.7 & 3.623 & 0.767 & 1.102 & 0.069 & Smail et al. (2004) \\
\hline SMMJ221806.77+001245.7 & 3.623 & 2.170 & 8.654 & 2.089 & Smail et al. (2004) \\
\hline SMMJ221806.77+001245.7 & 3.623 & 3.600 & 19.800 & 2.000 & Hainline (2008) \\
\hline SMMJ221806.77+001245.7 & 3.623 & 4.500 & 25.800 & 2.600 & Hainline (2008) \\
\hline SMMJ221806.77+001245.7 & 3.623 & 5.800 & 10.100 & 3.100 & Hainline (2008) \\
\hline SMMJ221806.77+001245.7 & 3.623 & 8.000 & 36.300 & 4.100 & Hainline (2008) \\
\hline SMMJ221806.77+001245.7 & 3.623 & 24.000 & 475.200 & 44.000 & Hainline (2008) \\
\hline SMMJ221806.77+001245.7 & 3.623 & 850.000 & 8400.000 & 2300.000 & Chapman et al. (2005) \\
\hline SMMJ221806.77+001245.7 & 3.623 & 214000.000 & 241.500 & 11.200 & Chapman et al. (2005) \\
\hline
\end{tabular}

Notes. The second reference in the first entry for each SMG indicates that there exists a mid-IR Spitzer/IRS spectrum of this object (Valiante et al. 2007; Pope et al. 2008; Menéndez-Delmestre et al. 2007; Menéndez-Delmestre et al. 2009).

References. Ivison et al. (2002, 2005); Chapman et al. (2003b, 2005); Capak et al. (2004); Clements et al. (2004); Egami et al. (2004); Greve et al. (2004); Smail et al. (2004); Fomalont et al. (2006); Kovács et al. (2006); Laurent et al. (2006); Tacconi et al. (2006); Pope et al. (2006); Huynh et al. (2007); Hainline (2008). 
Table A.2. Photometry upper limits of SMGs.

\begin{tabular}{|c|c|c|c|c|c|}
\hline SMG & $z$ & $\begin{array}{r}\lambda_{\mathrm{obs}} \\
(\mu \mathrm{m})\end{array}$ & $\begin{array}{r}\text { Flux } \\
(\mu \mathrm{Jy})\end{array}$ & $\begin{array}{l}\text { Error } \\
(\mu \mathrm{Jy})\end{array}$ & Reference \\
\hline SMMJ030227.73+000653.5 & 1.408 & 70.000 & 13600.000 & 0.000 & Hainline (2008) \\
\hline SMMJ030231.81+001031.3 & 1.316 & 0.365 & 0.059 & 0.000 & Clements et al. (2004) \\
\hline SMMJ030231.81+001031.3 & 1.316 & 0.428 & 0.100 & 0.000 & Chapman et al. (2005) \\
\hline SMMJ030231.81+001031.3 & 1.316 & 0.440 & 0.102 & 0.000 & Clements et al. (2004) \\
\hline SMMJ030231.81+001031.3 & 1.316 & 0.656 & 0.331 & 0.000 & Chapman et al. (2005) \\
\hline SMMJ030231.81+001031.3 & 1.316 & 0.767 & 0.806 & 0.000 & Smail et al. (2004) \\
\hline SMMJ030231.81+001031.3 & 1.316 & 0.767 & 0.205 & 0.000 & Clements et al. (2004) \\
\hline SMMJ030231.81+001031.3 & 1.316 & 2.170 & 2.865 & 0.000 & Smail et al. (2004) \\
\hline SMMJ030231.81+001031.3 & 1.316 & 2.170 & 2.655 & 0.000 & Clements et al. (2004) \\
\hline SMMJ030231.81+001031.3 & 1.316 & 3.600 & 0.700 & 0.000 & Hainline (2008) \\
\hline SMMJ030231.81+001031.3 & 1.316 & 5.800 & 5.500 & 0.000 & Hainline (2008) \\
\hline SMMJ030231.81+001031.3 & 1.316 & 8.000 & 3.500 & 0.000 & Hainline (2008) \\
\hline SMMJ030231.81+001031.3 & 1.316 & 24.000 & 110.700 & 0.000 & Hainline (2008) \\
\hline SMMJ030231.81+001031.3 & 1.316 & 70.000 & 14800.000 & 0.000 & Hainline (2008) \\
\hline SMMJ030236.15+000817.1 & 2.435 & 0.365 & 0.059 & 0.000 & Clements et al. (2004) \\
\hline SMMJ030236.15+000817.1 & 2.435 & 0.428 & 0.100 & 0.000 & Chapman et al. (2005) \\
\hline SMMJ030236.15+000817.1 & 2.435 & 0.440 & 0.102 & 0.000 & Clements et al. (2004) \\
\hline SMMJ030236.15+000817.1 & 2.435 & 0.767 & 0.205 & 0.000 & Clements et al. (2004) \\
\hline SMMJ030236.15+000817.1 & 2.435 & 0.767 & 0.806 & 0.000 & Smail et al. (2004) \\
\hline SMMJ030236.15+000817.1 & 2.435 & 1.250 & 4.809 & 0.000 & Smail et al. (2004) \\
\hline SMMJ030236.15+000817.1 & 2.435 & 2.170 & 2.865 & 0.000 & Smail et al. (2004) \\
\hline SMMJ030236.15+000817.1 & 2.435 & 8.000 & 3.500 & 0.000 & Hainline (2008) \\
\hline SMMJ030236.15+000817.1 & 2.435 & 24.000 & 78.200 & 0.000 & Hainline (2008) \\
\hline SMMJ030236.15+000817.1 & 2.435 & 70.000 & 10000.000 & 0.000 & Hainline (2008) \\
\hline SMMJ030238.62+001106.3 & 0.276 & 5.800 & 5.100 & 0.000 & Hainline (2008) \\
\hline SMMJ030238.62+001106.3 & 0.276 & 8.000 & 3.800 & 0.000 & Hainline (2008) \\
\hline SMMJ030238.62+001106.3 & 0.276 & 24.000 & 107.500 & 0.000 & Hainline (2008) \\
\hline SMMJ030238.62+001106.3 & 0.276 & 70.000 & 12700.000 & 0.000 & Hainline (2008) \\
\hline SMMJ030244.82+000632.3 & 0.176 & 5.800 & 2.600 & 0.000 & Hainline (2008) \\
\hline SMMJ030244.82+000632.3 & 0.176 & 24.000 & 83.700 & 0.000 & Hainline (2008) \\
\hline SMMJ030244.82+000632.3 & 0.176 & 70.000 & 10400.000 & 0.000 & Hainline (2008) \\
\hline SMMJ105151.69+572636.0 & 1.147 & 70.000 & 4200.000 & 0.000 & Hainline (2008) \\
\hline SMMJ105151.69+572636.0 & 1.147 & 61182.000 & 60.000 & 0.000 & Ivison et al. (2002) \\
\hline SMMJ105155.47+572312.7 & 2.686 & 70.000 & 3600.000 & 0.000 & Hainline (2008) \\
\hline SMMJ105158.02+571800.2 & 2.239 & 70.000 & 4100.000 & 0.000 & Hainline (2008) \\
\hline SMMJ105200.22+572420.2 & 0.689 & 24.000 & 65.100 & 0.000 & Hainline (2008) \\
\hline SMMJ105201.25+572445.7 & 2.148 & 0.767 & 0.243 & 0.000 & Ivison et al. (2002) \\
\hline SMMJ105201.25+572445.7 & 2.148 & 70.000 & 3700.000 & 0.000 & Hainline (2008) \\
\hline SMMJ105207.49+571904.0 & 2.689 & 2.170 & 3.777 & 0.000 & Ivison et al. (2002) \\
\hline SMMJ105207.49+571904.0 & 2.689 & 2.170 & 4.541 & 0.000 & Smail et al. (2004) \\
\hline SMMJ105207.49+571904.0 & 2.689 & 5.800 & 4.800 & 0.000 & Hainline (2008) \\
\hline SMMJ105207.49+571904.0 & 2.689 & 8.000 & 5.600 & 0.000 & Hainline (2008) \\
\hline SMMJ105207.49+571904.0 & 2.689 & 70.000 & 3200.000 & 0.000 & Hainline (2008) \\
\hline SMMJ105207.49+571904.0 & 2.689 & 1200.000 & 400.000 & 800.000 & Kovács et al. (2006) \\
\hline SMMJ105225.79+571906.4 & 2.372 & 70.000 & 3200.000 & 0.000 & Hainline (2008) \\
\hline SMMJ105227.58+572512.4 & 2.142 & 70.000 & 4500.000 & 0.000 & Hainline (2008) \\
\hline SMMJ105227.77+572218.2 & 1.956 & 0.656 & 0.034 & 0.000 & Ivison et al. (2005) \\
\hline SMMJ105227.77+572218.2 & 1.956 & 0.767 & 0.243 & 0.000 & Smail et al. (2004) \\
\hline SMMJ105227.77+572218.2 & 1.956 & 2.170 & 4.142 & 0.000 & Smail et al. (2004) \\
\hline SMMJ105227.77+572218.2 & 1.956 & 3.600 & 0.500 & 0.000 & Hainline (2008) \\
\hline SMMJ105227.77+572218.2 & 1.956 & 4.500 & 0.300 & 0.000 & Hainline (2008) \\
\hline SMMJ105227.77+572218.2 & 1.956 & 5.800 & 4.900 & 0.000 & Hainline (2008) \\
\hline SMMJ105227.77+572218.2 & 1.956 & 8.000 & 1.800 & 0.000 & Hainline (2008) \\
\hline SMMJ105227.77+572218.2 & 1.956 & 24.000 & 63.900 & 0.000 & Hainline (2008) \\
\hline SMMJ105227.77+572218.2 & 1.956 & 70.000 & 4100.000 & 0.000 & Hainline (2008) \\
\hline SMMJ105227.77+572218.2 & 1.956 & 350.000 & 11300.000 & 6700.000 & Kovács et al. (2006) \\
\hline SMMJ105230.73+572209.5 & 2.611 & 70.000 & 4100.000 & 0.000 & Hainline (2008) \\
\hline SMMJ105238.19+571651.1 & 1.852 & 8.000 & 7.600 & 0.000 & Hainline (2008) \\
\hline SMMJ105238.19+571651.1 & 1.852 & 70.000 & 3900.000 & 0.000 & Hainline (2008) \\
\hline
\end{tabular}


Table A.2. continued.

\begin{tabular}{|c|c|c|c|c|c|}
\hline SMG & $z$ & $\begin{array}{r}\lambda_{\text {obs }} \\
(\mu \mathrm{m})\end{array}$ & $\begin{array}{c}\text { Flux } \\
(\mu \mathrm{Jy})\end{array}$ & $\begin{array}{l}\text { Error } \\
(\mu \mathrm{Jy})\end{array}$ & Reference \\
\hline SMMJ105238.30+572435.8 & 3.036 & 8.000 & 57.200 & 0.000 & Hainline (2008) \\
\hline SMMJ105238.30+572435.8 & 3.036 & 70.000 & 6400.000 & 0.000 & Hainline (2008) \\
\hline SMMJ105238.30+572435.8 & 3.036 & 61182.000 & 226.000 & 0.000 & Ivison et al. (2002) \\
\hline SMMJ123549.44+621536.8 & 2.203 & 4.500 & 0.200 & 0.000 & Hainline (2008) \\
\hline SMMJ123549.44+621536.8 & 2.203 & 8.000 & 1.500 & 0.000 & Hainline (2008) \\
\hline SMMJ123549.44+621536.8 & 2.203 & 70.000 & 4100.000 & 0.000 & Hainline (2008) \\
\hline SMMJ123553.26+621337.7 & 2.098 & 1.895 & -0.244 & 1.228 & Capak et al. (2004) \\
\hline SMMJ123553.26+621337.7 & 2.098 & 24.000 & 70.900 & 0.000 & Hainline (2008) \\
\hline SMMJ123553.26+621337.7 & 2.098 & 70.000 & 2100.000 & 0.000 & Hainline (2008) \\
\hline SMMJ123555.14+620901.7 & 1.875 & 70.000 & 2000.000 & 0.000 & ? \\
\hline SMMJ123600.10+620253.5 & 2.710 & 70.000 & 5500.000 & 0.000 & Hainline (2008) \\
\hline SMMJ123600.15+621047.2 & 1.994 & 0.443 & 0.043 & 0.021 & Capak et al. (2004) \\
\hline SMMJ123600.15+621047.2 & 1.994 & 0.547 & 0.030 & 0.016 & Capak et al. (2004) \\
\hline SMMJ123600.15+621047.2 & 1.994 & 0.907 & 0.030 & 0.046 & Capak et al. (2004) \\
\hline SMMJ123600.15+621047.2 & 1.994 & 1.895 & -2.029 & 0.928 & Capak et al. (2004) \\
\hline SMMJ123600.15+621047.2 & 1.994 & 70.000 & 3800.000 & 0.000 & Hainline (2008) \\
\hline SMMJ123606.72+621550.7 & 2.416 & 0.767 & 0.128 & 0.000 & Smail et al. (2004) \\
\hline SMMJ123606.72+621550.7 & 2.416 & 1.250 & 1.593 & 0.000 & Smail et al. (2004) \\
\hline SMMJ123606.72+621550.7 & 2.416 & 1.895 & 7.125 & 3.028 & Capak et al. (2004) \\
\hline SMMJ123606.72+621550.7 & 2.416 & 70.000 & 3200.000 & 0.000 & Hainline (2008) \\
\hline SMMJ123606.85+621021.4 & 2.509 & 24.000 & 113.500 & 0.000 & Hainline (2008) \\
\hline SMMJ123606.85+621021.4 & 2.509 & 70.000 & 3900.000 & 0.000 & Hainline (2008) \\
\hline SMMJ123616.15+621513.7 & 2.578 & 0.365 & 0.028 & 0.023 & Capak et al. (2004) \\
\hline SMMJ123616.15+621513.7 & 2.578 & 1.895 & 1.741 & 0.995 & Capak et al. (2004) \\
\hline SMMJ123616.15+621513.7 & 2.578 & 70.000 & 2000.000 & 0.000 & Hainline (2008) \\
\hline SMMJ123618.33+621550.5 & 1.865 & 1.895 & 2.811 & 0.990 & Capak et al. (2004) \\
\hline SMMJ123618.33+621550.5 & 1.865 & 70.000 & 3600.000 & 0.000 & Hainline (2008) \\
\hline SMMJ123621.27+621708.4 & 1.998 & 1.250 & 1.593 & 0.000 & Smail et al. (2004) \\
\hline SMMJ123621.27+621708.4 & 1.988 & 70.000 & 2200.000 & 0.000 & Hainline (2008) \\
\hline SMMJ123622.65+621629.7 & 2.466 & 0.775 & 0.023 & 0.000 & Pope et al. (2006) \\
\hline SMMJ123622.65+621629.7 & 2.466 & 1.250 & 1.593 & 0.000 & Smail et al. (2004) \\
\hline SMMJ123622.65+621629.7 & 2.466 & 1.895 & 2.170 & 0.832 & Capak et al. (2004) \\
\hline SMMJ123622.65+621629.7 & 2.466 & 70.000 & 2700.000 & 0.000 & Hainline (2008) \\
\hline SMMJ123629.13+621045.8 & 1.013 & 70.000 & 1800.000 & 0.000 & Hainline (2008) \\
\hline SMMJ123632.61+620800.1 & 1.993 & 70.000 & 2400.000 & 0.000 & Hainline (2008) \\
\hline SMMJ123634.51+621241.0 & 1.219 & $1.0000000 \mathrm{e}+10$ & $1.0000000 \mathrm{e}+13$ & 0.000 & $? ?$ \\
\hline SMMJ123635.59+621424.1 & 2.005 & 70.000 & 2800.000 & 0.000 & Hainline (2008) \\
\hline SMMJ123636.75+621156.1 & 0.557 & 24.000 & 127.800 & 0.000 & Hainline (2008) \\
\hline SMMJ123636.75+621156.1 & 0.557 & 70.000 & 5300.000 & 0.000 & Hainline (2008) \\
\hline SMMJ123651.76+621221.3 & 0.298 & 70.000 & 1600.000 & 0.000 & Hainline (2008) \\
\hline SMMJ123707.21+621408.1 & 2.484 & 0.365 & 0.035 & 0.023 & Capak et al. (2004) \\
\hline SMMJ123707.21+621408.1 & 2.484 & 70.000 & 1600.000 & 0.000 & Hainline (2008) \\
\hline SMMJ123707.21+621408.1 & 2.484 & 1300.000 & 1400.000 & 0.000 & Tacconi et al. (2006) \\
\hline SMMJ123711.98+621325.7 & 1.992 & 1.250 & 1.593 & 0.000 & Smail et al. (2004) \\
\hline SMMJ123711.98+621325.7 & 1.992 & 1.895 & 2.498 & 1.119 & Capak et al. (2004) \\
\hline SMMJ123711.98+621325.7 & 1.992 & 70.000 & 1500.000 & 0.000 & Hainline (2008) \\
\hline SMMJ123712.05+621212.3 & 2.914 & 0.365 & 0.050 & 0.027 & Capak et al. (2004) \\
\hline SMMJ123712.05+621212.3 & 2.914 & 0.798 & 0.131 & 0.056 & Capak et al. (2004) \\
\hline SMMJ123712.05+621212.3 & 2.914 & 0.907 & 0.128 & 0.070 & Capak et al. (2004) \\
\hline SMMJ123712.05+621212.3 & 2.914 & 1.250 & 1.593 & 0.000 & Smail et al. (2004) \\
\hline SMMJ123712.05+621212.3 & 2.914 & 1.895 & -0.846 & 0.977 & Capak et al. (2004) \\
\hline SMMJ123712.05+621212.3 & 2.914 & 70.000 & 1500.000 & 0.000 & Hainline (2008) \\
\hline SMMJ123716.01+620323.3 & 2.037 & 70.000 & 6000.000 & 0.000 & Hainline (2008) \\
\hline SMMJ123721.87+621035.3 & 0.979 & 70.000 & 3000.000 & 0.000 & Hainline (2008) \\
\hline SMMJ131201.17+424208.1 & 3.405 & $1.0000000 \mathrm{e}+10$ & $1.0000000 \mathrm{e}+13$ & 0.000 & $? ?$ \\
\hline SMMJ131208.82+424129.1 & 1.544 & $1.0000000 \mathrm{e}+10$ & $1.0000000 \mathrm{e}+13$ & 0.000 & $? ?$ \\
\hline SMMJ131212.69+424422.5 & 2.805 & 0.428 & 0.058 & 0.000 & Chapman et al. (2005) \\
\hline SMMJ131212.69+424422.5 & 2.805 & 0.630 & 0.078 & 0.000 & Fomalont et al. (2006) \\
\hline SMMJ131212.69+424422.5 & 2.805 & 0.767 & 0.243 & 0.000 & Smail et al. (2004) \\
\hline SMMJ131212.69+424422.5 & 2.805 & 0.920 & 0.140 & 0.000 & Fomalont et al. (2006) \\
\hline
\end{tabular}


Table A.2. continued.

\begin{tabular}{|c|c|c|c|c|c|}
\hline SMG & $z$ & $\begin{array}{r}\lambda_{\text {obs }} \\
(\mu \mathrm{m})\end{array}$ & $\begin{array}{l}\text { Flux } \\
(\mu \mathrm{Jy})\end{array}$ & $\begin{array}{l}\text { Error } \\
(\mu \mathrm{Jy})\end{array}$ & Reference \\
\hline SMMJ131212.69+424422.5 & 2.805 & 1.250 & 2.524 & 0.000 & Smail et al. (2004) \\
\hline SMMJ131212.69+424422.5 & 2.805 & 5.800 & 8.200 & 0.000 & Hainline (2008) \\
\hline SMMJ131212.69+424422.5 & 2.805 & 8.000 & 10.200 & 0.000 & Hainline (2008) \\
\hline SMMJ131212.69+424422.5 & 2.805 & 350.000 & 3700.000 & 4400.000 & Kovács et al. (2006) \\
\hline SMMJ131215.27+423900.9 & 2.565 & $1.0000000 \mathrm{e}+10$ & $1.0000000 \mathrm{e}+13$ & 0.000 & $? ?$ \\
\hline SMMJ131222.35+423814.1 & 2.565 & $1.0000000 \mathrm{e}+10$ & $1.0000000 \mathrm{e}+13$ & 0.000 & $? ?$ \\
\hline SMMJ131225.20+424344.5 & 1.038 & $1.0000000 \mathrm{e}+10$ & $1.0000000 \mathrm{e}+13$ & 0.000 & $? ?$ \\
\hline SMMJ131225.73+423941.4 & 1.554 & 5.800 & 4.900 & 0.000 & Hainline (2008) \\
\hline SMMJ131225.73+423941.4 & 1.554 & 8.000 & 5.700 & 0.000 & Hainline (2008) \\
\hline SMMJ131225.73+423941.4 & 1.554 & 350.000 & 14700.000 & 7400.000 & Kovács et al. (2006) \\
\hline SMMJ131228.30+424454.8 & 2.931 & 2.170 & 3.445 & 0.000 & Smail et al. (2004) \\
\hline SMMJ131231.07+424609.0 & 2.713 & 0.428 & 0.058 & 0.000 & Chapman et al. (2005) \\
\hline SMMJ131231.07+424609.0 & 2.713 & 1.250 & 2.524 & 0.000 & Smail et al. (2004) \\
\hline SMMJ131231.07+424609.0 & 2.713 & 2.170 & 3.445 & 0.000 & Smail et al. (2004) \\
\hline SMMJ131231.07+424609.0 & 2.713 & 3.600 & 0.600 & 0.000 & Hainline (2008) \\
\hline SMMJ131231.07+424609.0 & 2.713 & 4.500 & 1.000 & 0.000 & Hainline (2008) \\
\hline SMMJ131231.07+424609.0 & 2.713 & 5.800 & 5.100 & 0.000 & Hainline (2008) \\
\hline SMMJ131231.07+424609.0 & 2.713 & 8.000 & 6.300 & 0.000 & Hainline (2008) \\
\hline SMMJ131232.31+423949.5 & 2.320 & $1.0000000 \mathrm{e}+10$ & $1.0000000 \mathrm{e}+13$ & 0.000 & ?? \\
\hline SMMJ131239.14+424155.7 & 2.242 & 1.250 & 0.481 & 0.000 & Smail et al. (2004) \\
\hline SMMJ131239.14+424155.7 & 2.242 & 5.800 & 7.700 & 0.000 & Hainline (2008) \\
\hline SMMJ141741.81+522823.0 & 1.150 & $1.0000000 \mathrm{e}+10$ & $1.0000000 \mathrm{e}+13$ & 0.000 & $? ?$ \\
\hline SMMJ141742.04+523025.7 & 0.661 & $1.0000000 \mathrm{e}+10$ & $1.0000000 \mathrm{e}+13$ & 0.000 & $? ?$ \\
\hline SMMJ141750.50+523101.0 & 2.128 & 0.767 & 0.423 & 0.000 & Smail et al. (2004) \\
\hline SMMJ141750.50+523101.0 & 2.128 & 1.250 & 2.302 & 0.000 & Smail et al. (2004) \\
\hline SMMJ141750.50+523101.0 & 2.128 & 3.600 & 0.300 & 0.000 & Hainline (2008) \\
\hline SMMJ141750.50+523101.0 & 2.128 & 4.500 & 0.300 & 0.000 & Hainline (2008) \\
\hline SMMJ141750.50+523101.0 & 2.128 & 5.800 & 1.300 & 0.000 & Hainline (2008) \\
\hline SMMJ141750.50+523101.0 & 2.128 & 8.000 & 1.800 & 0.000 & Hainline (2008) \\
\hline SMMJ141750.50+523101.0 & 2.128 & 24.000 & 88.800 & 0.000 & Hainline (2008) \\
\hline SMMJ141750.50+523101.0 & 2.128 & 70.000 & 6700.000 & 0.000 & Hainline (2008) \\
\hline SMMJ141800.40+512820.3 & 1.913 & $1.0000000 \mathrm{e}+10$ & $1.0000000 \mathrm{e}+13$ & 0.000 & $? ?$ \\
\hline SMMJ141802.87+523011.1 & 2.127 & 0.767 & 0.423 & 0.000 & Smail et al. (2004) \\
\hline SMMJ141802.87+523011.1 & 2.127 & 8.000 & 2.000 & 0.000 & Hainline (2008) \\
\hline SMMJ141802.87+523011.1 & 2.127 & 24.000 & 93.200 & 0.000 & Hainline (2008) \\
\hline SMMJ141802.87+523011.1 & 2.127 & 70.000 & 6900.000 & 0.000 & Hainline (2008) \\
\hline SMMJ141809.00+522803.8 & 2.712 & 0.767 & 0.423 & 0.000 & Smail et al. (2004) \\
\hline SMMJ141809.00+522803.8 & 2.712 & 24.000 & 142.000 & 0.000 & Hainline (2008) \\
\hline SMMJ141809.00+522803.8 & 2.712 & 70.000 & 13400.000 & 0.000 & Hainline (2008) \\
\hline SMMJ141813.54+522923.4 & 3.484 & 1.250 & 2.302 & 0.000 & Smail et al. (2004) \\
\hline SMMJ141813.54+522923.4 & 3.484 & 3.600 & 0.200 & 0.000 & Hainline (2008) \\
\hline SMMJ141813.54+522923.4 & 3.484 & 4.500 & 0.500 & 0.000 & Hainline (2008) \\
\hline SMMJ141813.54+522923.4 & 3.484 & 5.800 & 2.100 & 0.000 & Hainline (2008) \\
\hline SMMJ141813.54+522923.4 & 3.484 & 8.000 & 3.800 & 0.000 & Hainline (2008) \\
\hline SMMJ141813.54+522923.4 & 3.484 & 24.000 & 169.000 & 0.000 & Hainline (2008) \\
\hline SMMJ141813.54+522923.4 & 3.484 & 70.000 & 9000.001 & 0.000 & Hainline (2008) \\
\hline SMMJ163627.94+405811.2 & 3.180 & 5.800 & 3.800 & 0.000 & Hainline (2008) \\
\hline SMMJ163627.94+405811.2 & 3.180 & 8.000 & 4.100 & 0.000 & Hainline (2008) \\
\hline SMMJ163627.94+405811.2 & 3.180 & 24.000 & 123.800 & 0.000 & Hainline (2008) \\
\hline SMMJ163627.94+405811.2 & 3.180 & 70.000 & 6700.000 & 0.000 & Hainline (2008) \\
\hline SMMJ163631.47+405546.9 & 2.283 & 0.550 & 0.161 & 0.000 & Ivison et al. (2002) \\
\hline SMMJ163631.47+405546.9 & 2.283 & 0.656 & 0.124 & 0.000 & Ivison et al. (2002) \\
\hline SMMJ163631.47+405546.9 & 2.283 & 0.767 & 0.243 & 0.000 & Ivison et al. (2002) \\
\hline SMMJ163631.47+405546.9 & 2.283 & 0.767 & 0.106 & 0.000 & Smail et al. (2004) \\
\hline SMMJ163631.47+405546.9 & 2.283 & 2.170 & 4.142 & 0.000 & Smail et al. (2004) \\
\hline SMMJ163631.47+405546.9 & 2.283 & 70.000 & 9400.000 & 0.000 & Hainline (2008) \\
\hline SMMJ163631.47+405546.9 & 2.283 & 1200.000 & 1100.000 & 700.000 & Kovács et al. (2006) \\
\hline SMMJ163639.01+405635.9 & 1.495 & 70.000 & 7800.000 & 0.000 & Hainline (2008) \\
\hline SMMJ163650.43+405734.5 & 2.378 & 70.000 & 9100.000 & 0.000 & Hainline (2008) \\
\hline SMMJ163658.19+410523.8 & 2.454 & 0.550 & 0.161 & 0.000 & Ivison et al. (2002) \\
\hline
\end{tabular}


Table A.2. continued.

\begin{tabular}{|c|c|c|c|c|c|}
\hline SMG & $z$ & $\begin{array}{r}\lambda_{\text {obs }} \\
(\mu \mathrm{m})\end{array}$ & $\begin{array}{r}\text { Flux } \\
(\mu \mathrm{Jy})\end{array}$ & $\begin{array}{l}\text { Error } \\
(\mu \mathrm{Jy})\end{array}$ & Reference \\
\hline SMMJ163658.19+410523.8 & 2.454 & 70.000 & 7900.000 & 0.000 & Hainline (2008) \\
\hline SMMJ163658.78+405728.1 & 1.190 & 70.000 & 6000.000 & 0.000 & Hainline (2008) \\
\hline SMMJ163704.34+410530.3 & 0.840 & 5.800 & 5.700 & 0.000 & Hainline (2008) \\
\hline SMMJ163704.34+410530.3 & 0.840 & 8.000 & 5.500 & 0.000 & Hainline (2008) \\
\hline SMMJ163704.34+410530.3 & 0.840 & 24.000 & 174.700 & 0.000 & Hainline (2008) \\
\hline SMMJ163704.34+410530.3 & 0.840 & 70.000 & 11600.000 & 0.000 & Hainline (2008) \\
\hline SMMJ163704.34+410530.3 & 0.840 & 1200.000 & 800.000 & 1100.000 & Kovács et al. (2006) \\
\hline SMMJ163706.51+405313.8 & 2.374 & 70.000 & 9500.001 & 0.000 & Hainline (2008) \\
\hline SMMJ221724.69+001242.1 & 0.510 & $1.0000000 \mathrm{e}+10$ & $1.0000000 \mathrm{e}+13$ & 0.000 & $? ?$ \\
\hline SMMJ221725.97+001238.9 & 3.094 & 0.428 & 0.058 & 0.000 & Chapman et al. (2005) \\
\hline SMMJ221725.97+001238.9 & 3.094 & 1.250 & 1.593 & 0.000 & Smail et al. (2004) \\
\hline SMMJ221725.97+001238.9 & 3.094 & 2.170 & 1.982 & 0.000 & Smail et al. (2004) \\
\hline SMMJ221725.97+001238.9 & 3.094 & 24.000 & 113.900 & 0.000 & Hainline (2008) \\
\hline SMMJ221725.97+001238.9 & 3.094 & 70.000 & 6300.000 & 0.000 & Hainline (2008) \\
\hline SMMJ221733.02+000906.0 & 0.926 & $1.0000000 \mathrm{e}+10$ & $1.0000000 \mathrm{e}+13$ & 0.000 & $? ?$ \\
\hline SMMJ221733.12+001120.2 & 0.652 & 70.000 & 10900.000 & 0.000 & Hainline (2008) \\
\hline SMMJ221733.91+001352.1 & 2.555 & 2.170 & 1.982 & 0.000 & Smail et al. (2004) \\
\hline SMMJ221733.91+001352.1 & 2.555 & 24.000 & 98.100 & 0.000 & Hainline (2008) \\
\hline SMMJ221733.91+001352.1 & 2.555 & 70.000 & 6200.000 & 0.000 & Hainline (2008) \\
\hline SMMJ221735.15+001537.2 & 3.098 & 5.800 & 1.900 & 0.000 & Hainline (2008) \\
\hline SMMJ221735.15+001537.2 & 3.098 & 24.000 & 112.100 & 0.000 & Hainline (2008) \\
\hline SMMJ221735.84+001558.9 & 3.089 & 24.000 & 132.800 & 0.000 & Hainline (2008) \\
\hline SMMJ221737.39+001025.1 & 2.614 & 24.000 & 176.700 & 0.000 & Hainline (2008) \\
\hline SMMJ221737.39+001025.1 & 2.614 & 70.000 & 12100.000 & 0.000 & Hainline (2008) \\
\hline SMMJ221804.42+002154.4 & 2.517 & 1.250 & 2.099 & 0.000 & Smail et al. (2004) \\
\hline SMMJ221804.42+002154.4 & 2.517 & 8.000 & 6.500 & 0.000 & Hainline (2008) \\
\hline SMMJ221804.42+002154.4 & 2.517 & 70.000 & 23800.000 & 0.000 & Hainline (2008) \\
\hline SMMJ221806.77+001245.7 & 3.623 & 1.250 & 6.340 & 0.000 & Smail et al. (2004) \\
\hline SMMJ221806.77+001245.7 & 3.623 & 70.000 & 18500.000 & 0.000 & Hainline (2008) \\
\hline
\end{tabular}

Notes. When the error is equal to zero, the flux column denotes $3 \sigma$ upper limit. Otherwise - formal flux at the position of an SMG.

"??" in the reference column means that there are no upper limits for this SMG. This line is added in order to preserve the same number of objects in all tables.

References. Ivison et al. (2002, 2005); Chapman et al. (2003b, 2005); Capak et al. (2004); Clements et al. (2004); Egami et al. (2004); Greve et al. (2004); Smail et al. (2004); Fomalont et al. (2006); Kovács et al. (2006); Laurent et al. (2006); Tacconi et al. (2006); Pope et al. (2006); Huynh et al. (2007); Hainline (2008). 
M. Michałowski et al.: Evolution of submillimeter galaxies

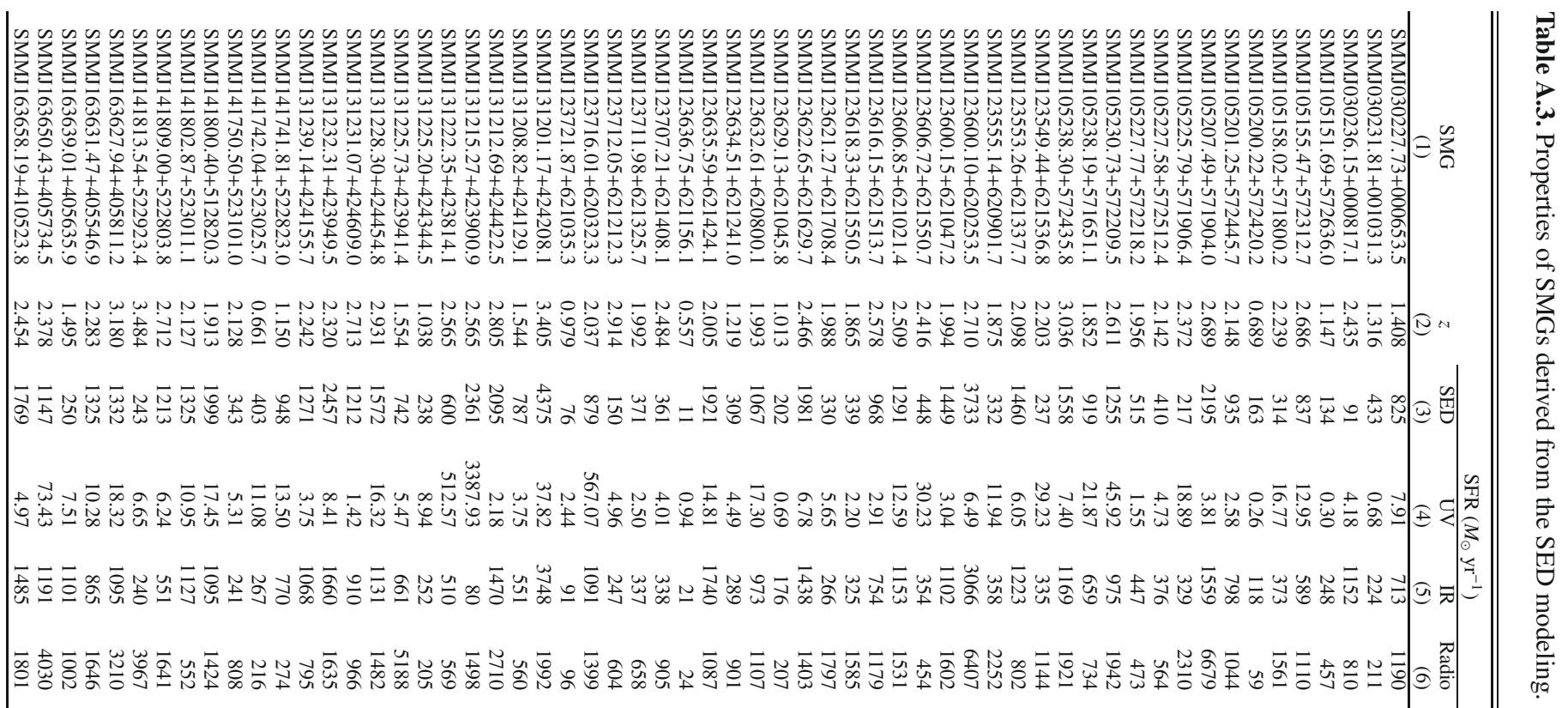

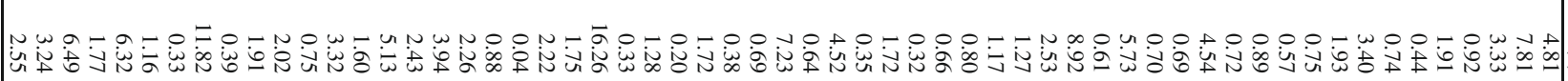

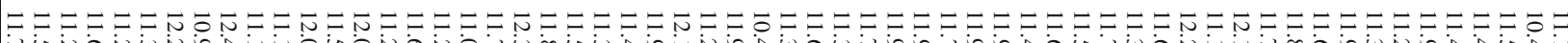

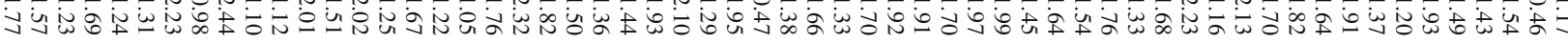

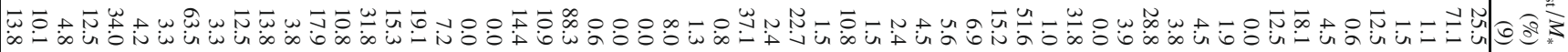

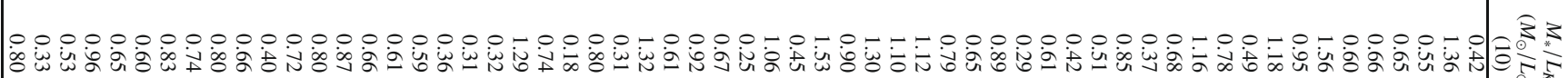

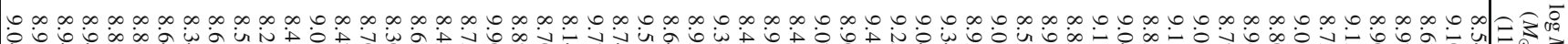

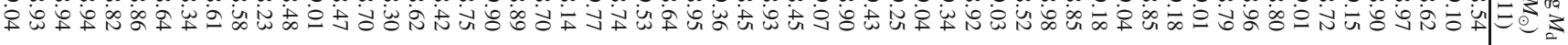

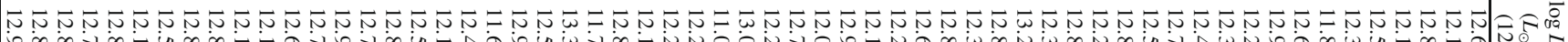
另

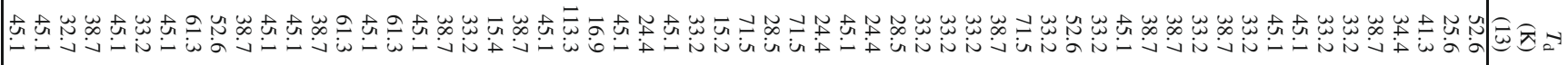

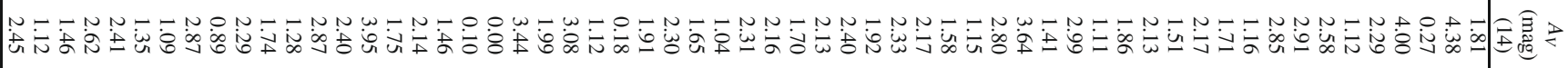

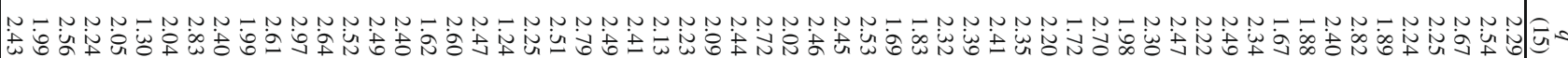

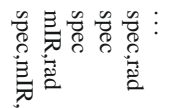
亮
范 :

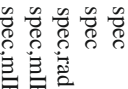

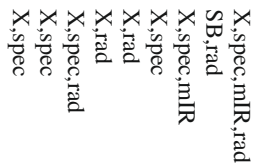

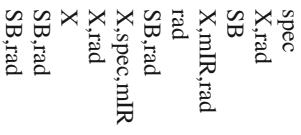

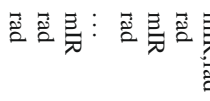




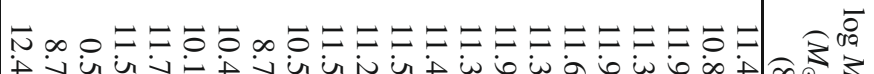

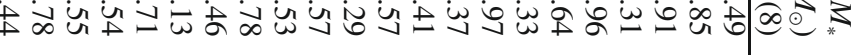

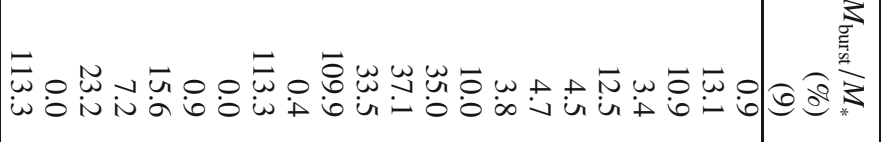

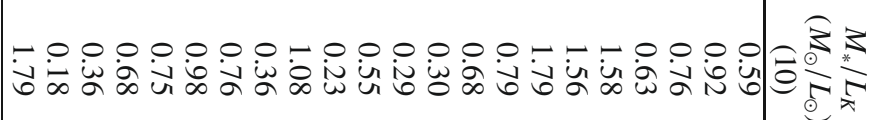

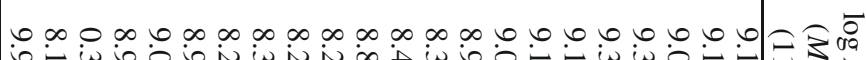

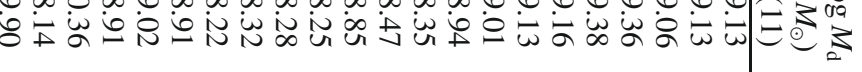

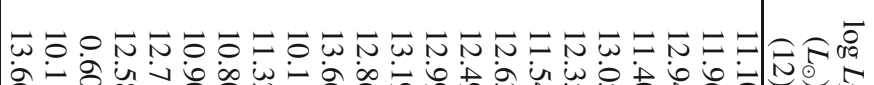

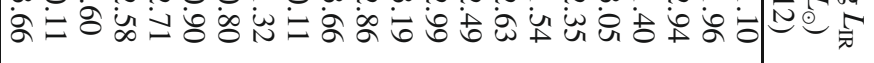

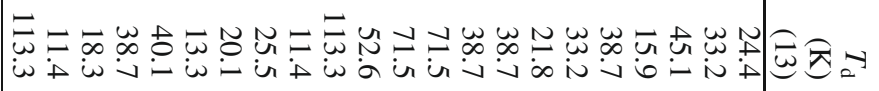

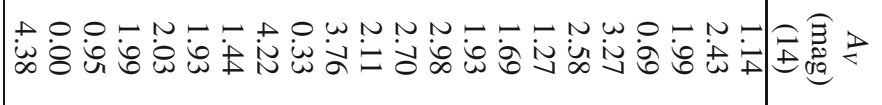

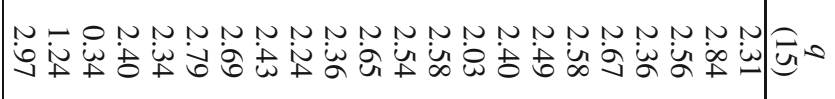

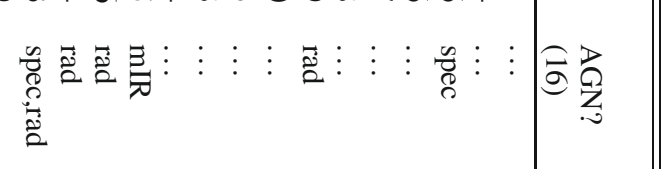




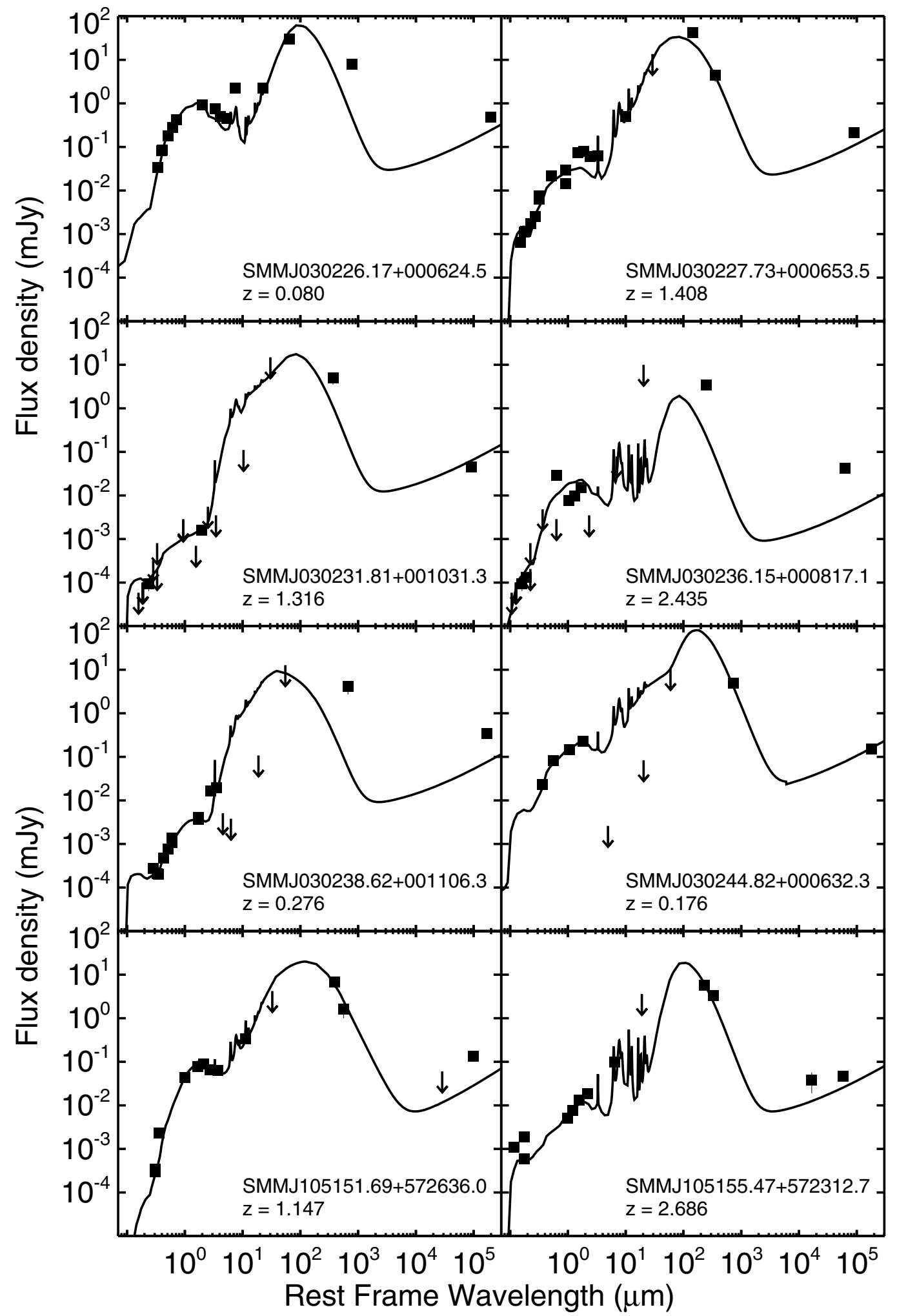

Fig. A.1. Spectral energy distributions (SEDs) of SMGs. Solid lines: the best GRASIL fits. Dashed lines: SEDs of GRB hosts (Michałowski et al. 2008) shown for comparison. Squares: detections with errors, in most cases, smaller than the size of the symbols. Arrows: $3 \sigma$ upper limit (values marked at the base). In the cases where our fits strongly underpredict the observed data at $850 \mu \mathrm{m}$, we adopted $L_{\mathrm{IR}}$ and $T_{\mathrm{d}}$ of Chapman et al. (2005). 


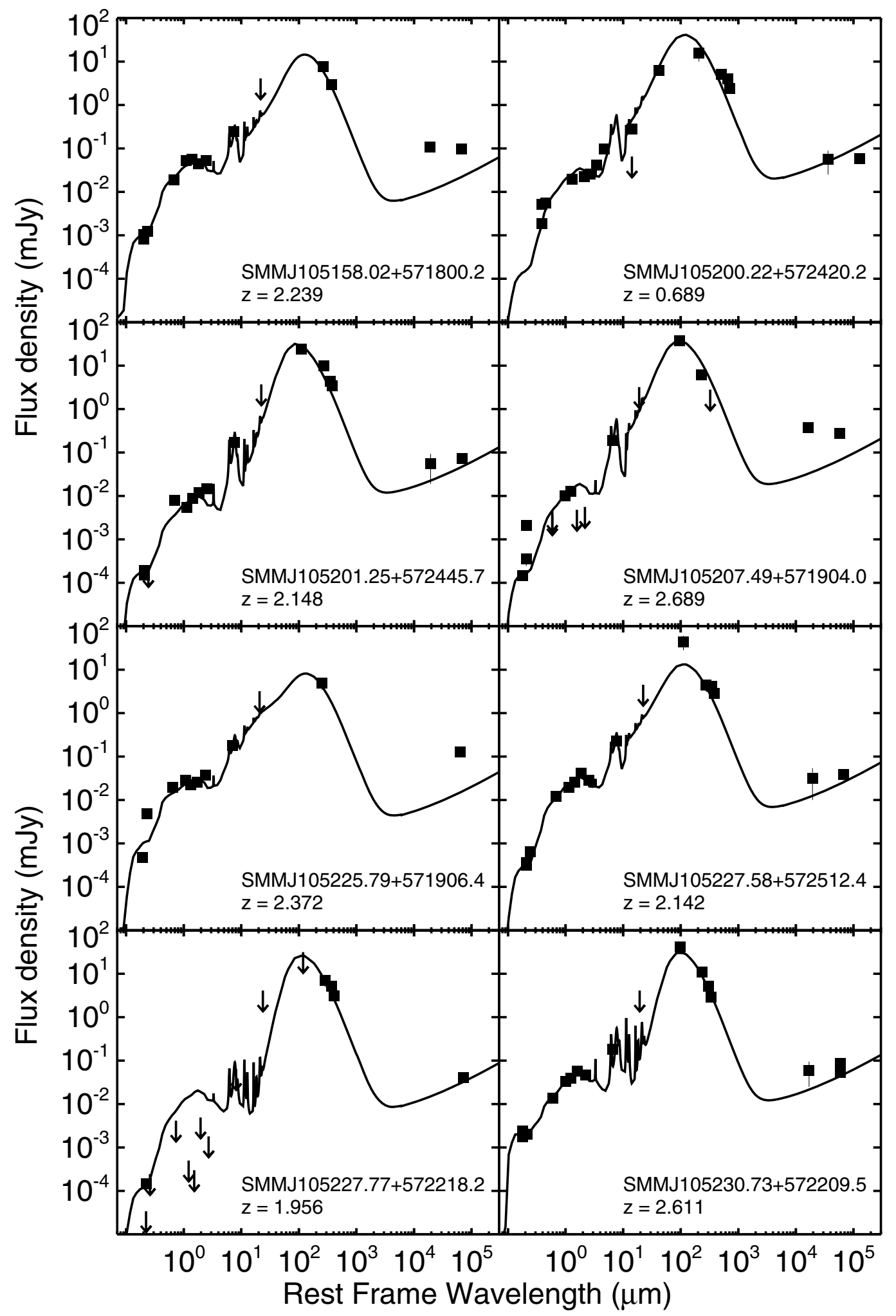

Fig. A.1. (continued). 
M. Michałowski et al.: Evolution of submillimeter galaxies

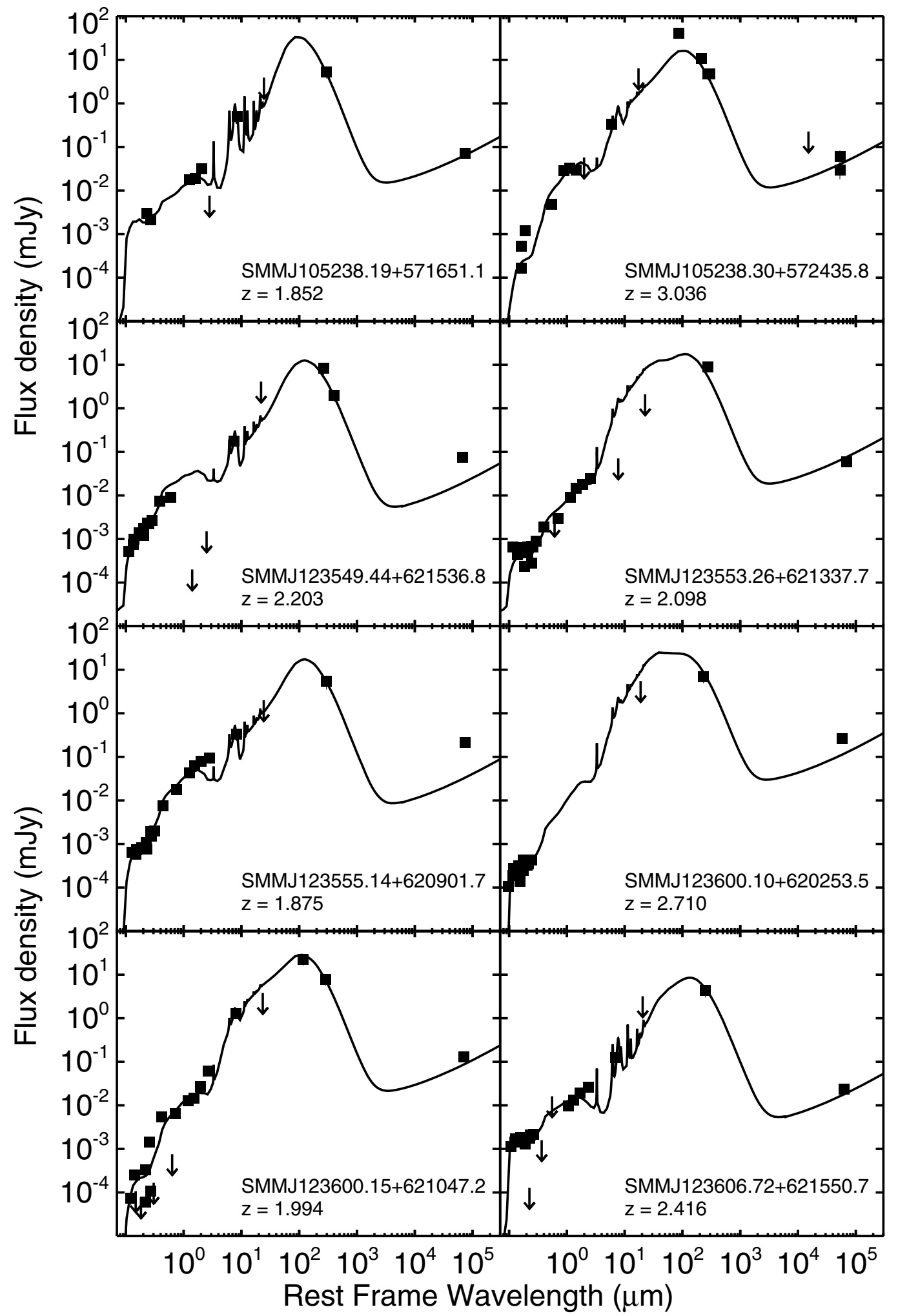

Fig. A.1. (continued). 


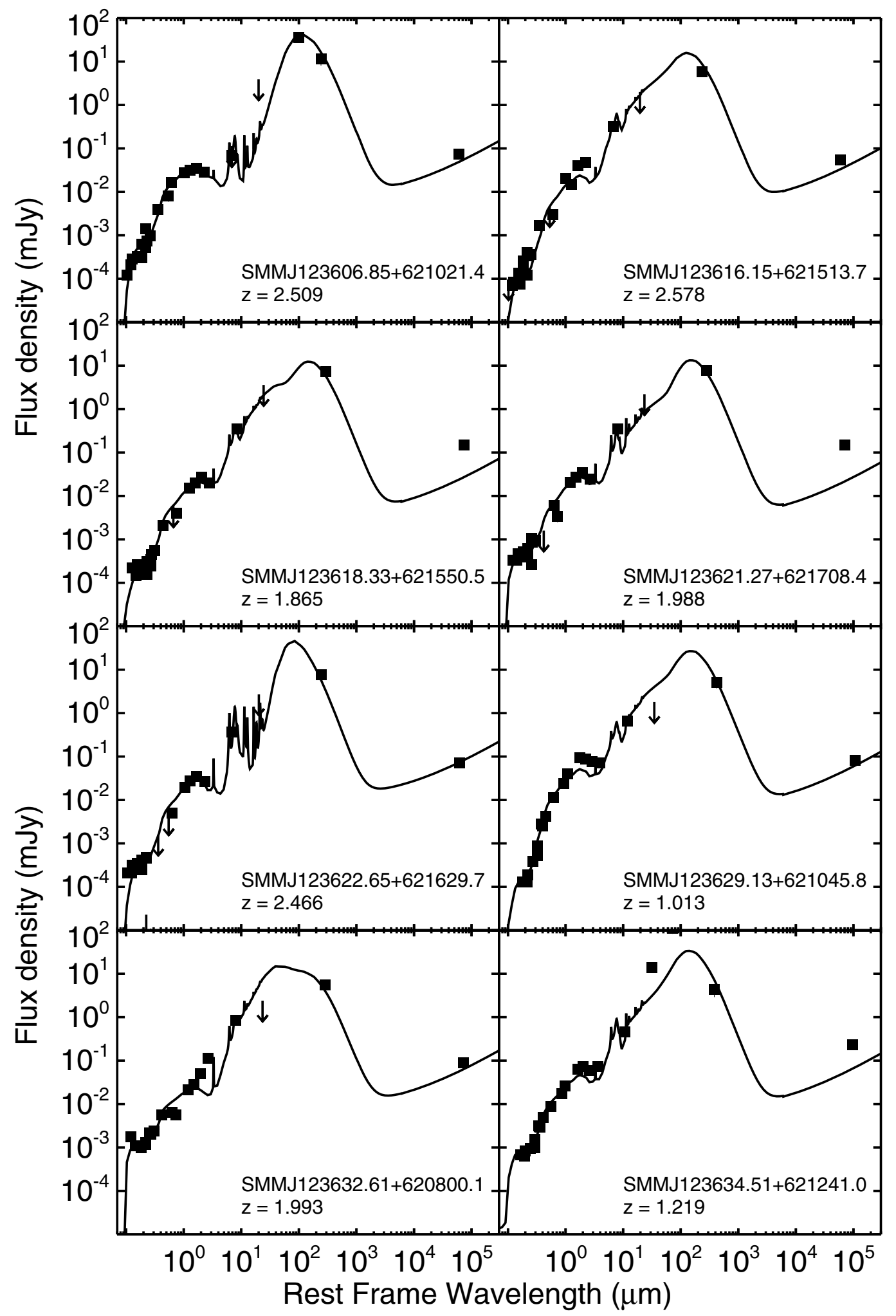

Fig. A.1. (continued). 
M. Michałowski et al.: Evolution of submillimeter galaxies

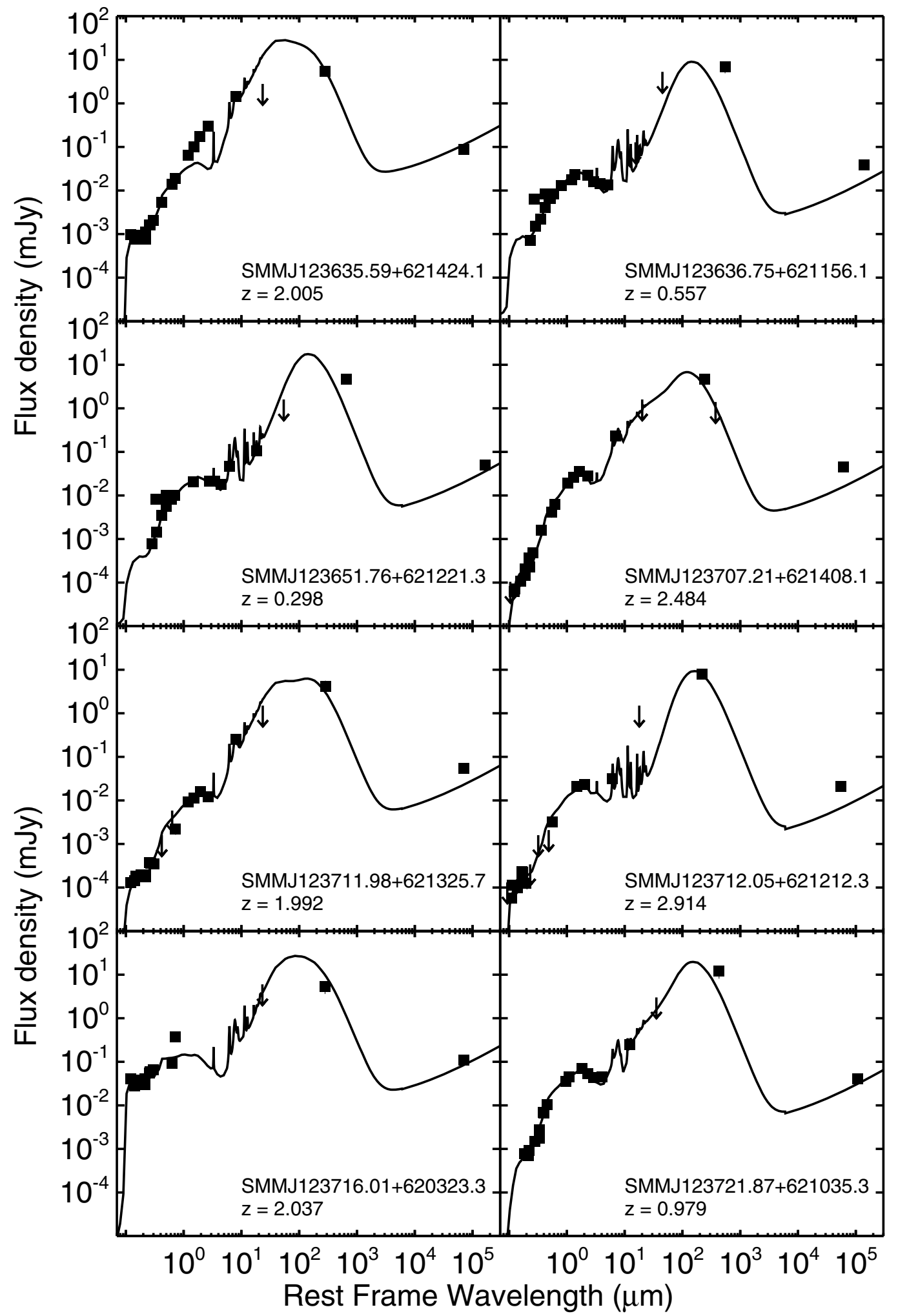

Fig. A.1. (continued). 


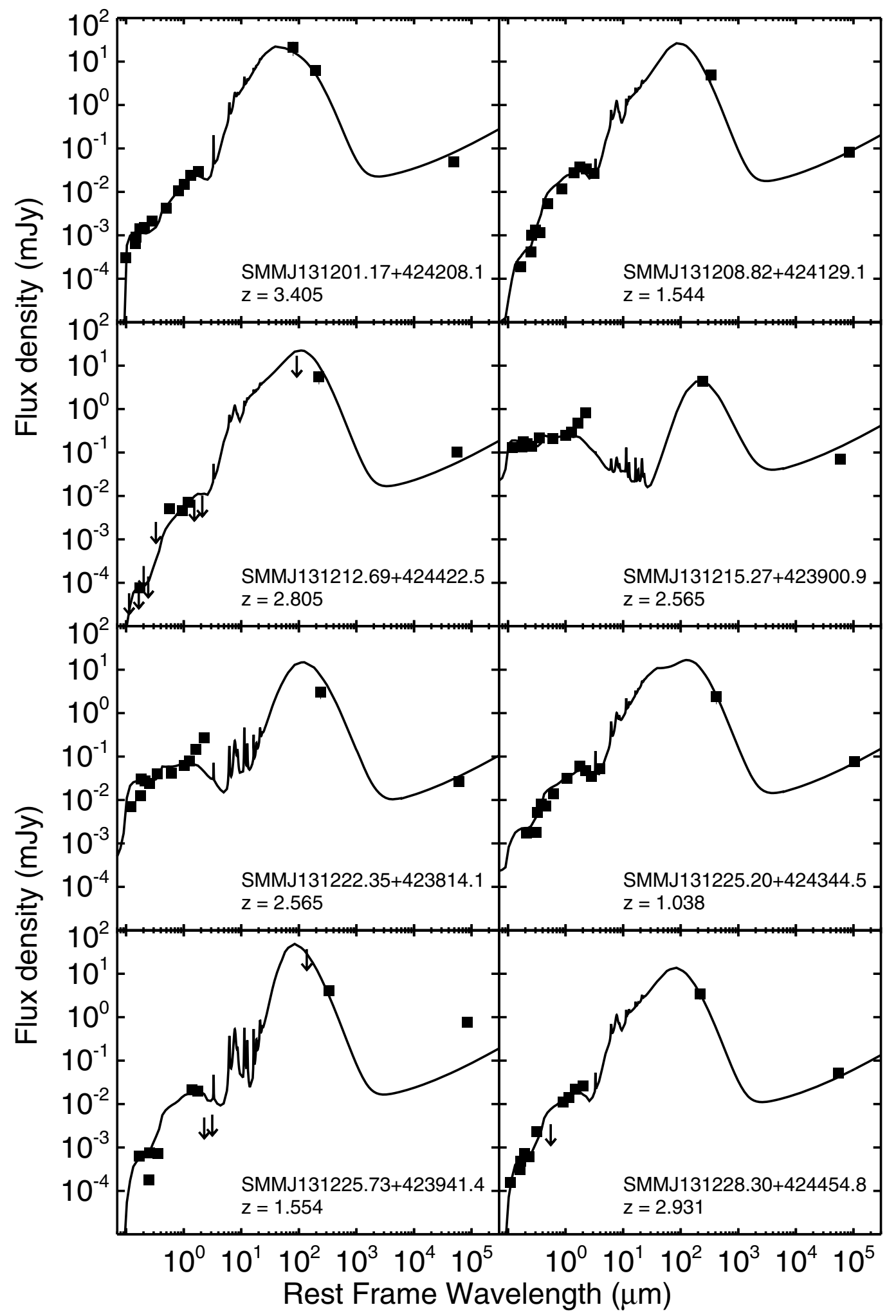

Fig. A.1. (continued). 
M. Michałowski et al.: Evolution of submillimeter galaxies

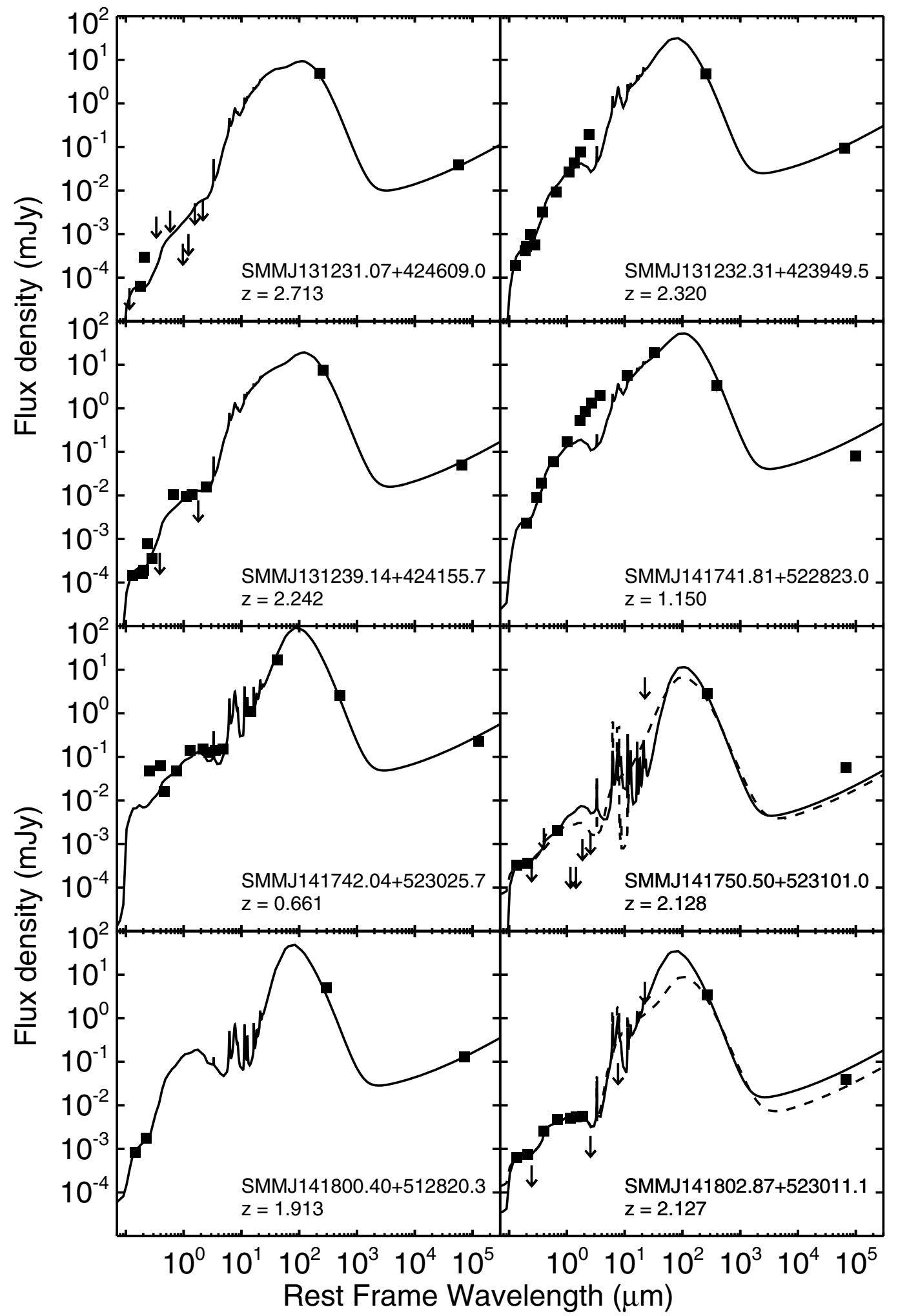

Fig. A.1. (continued). 


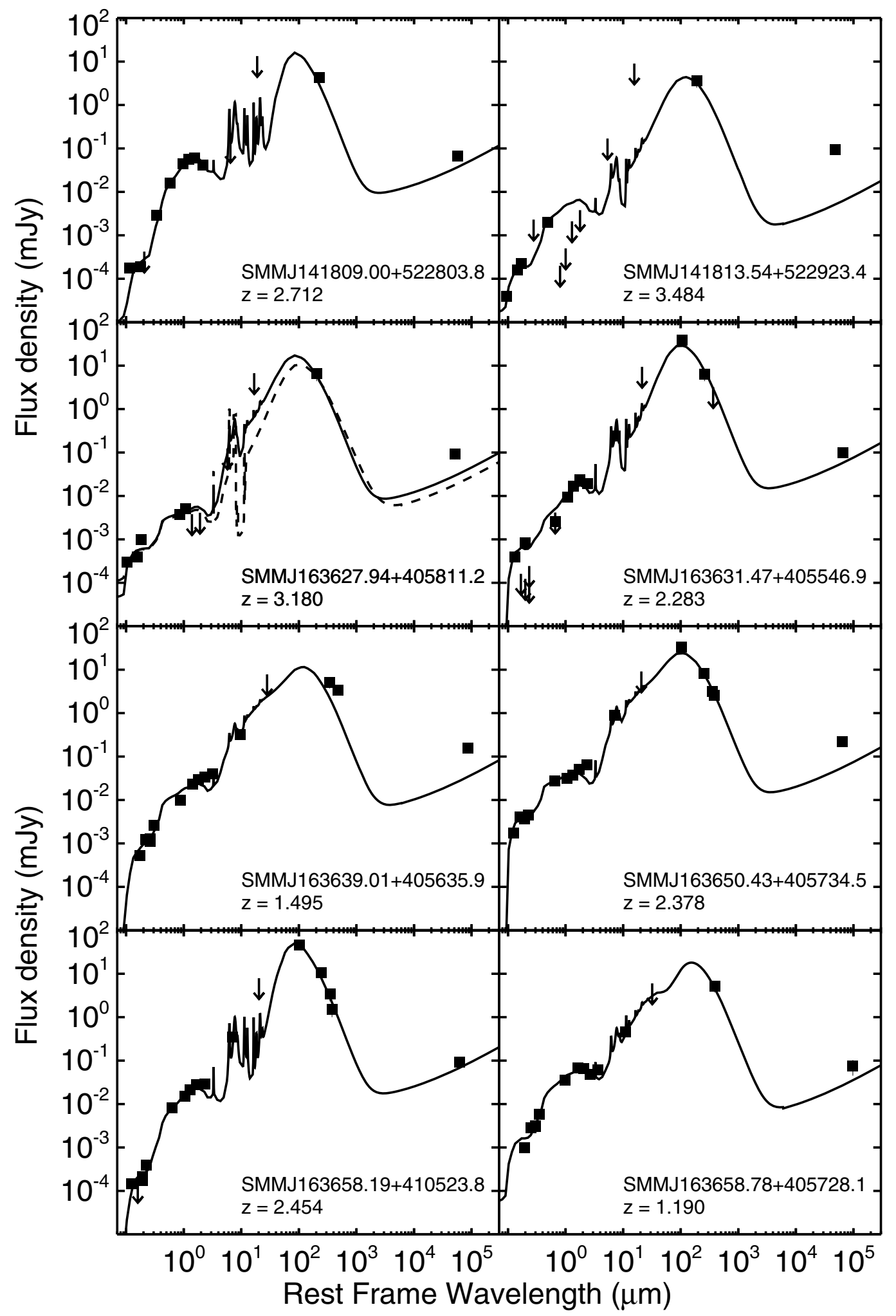

Fig. A.1. (continued). 
M. Michałowski et al.: Evolution of submillimeter galaxies

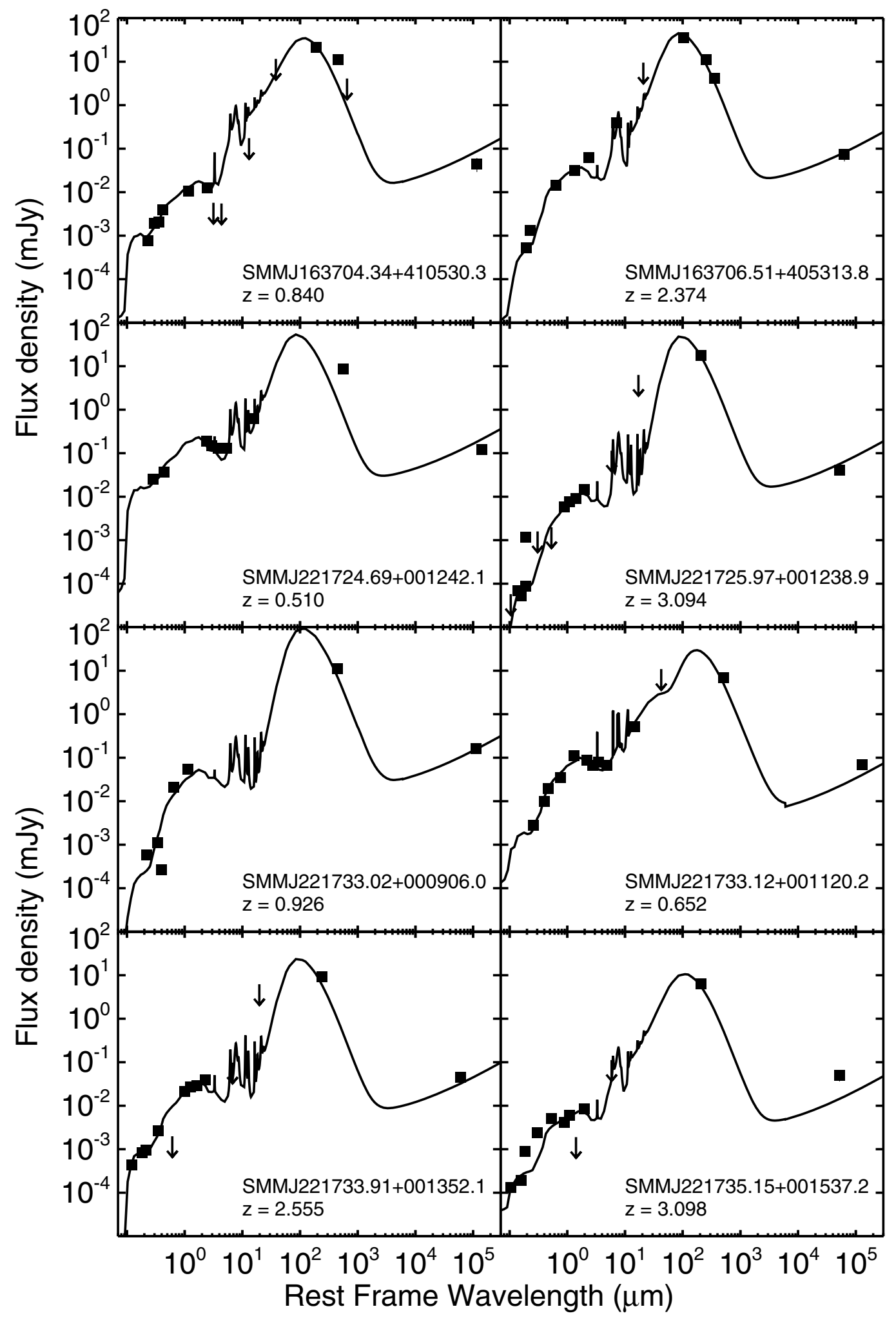

Fig. A.1. (continued). 


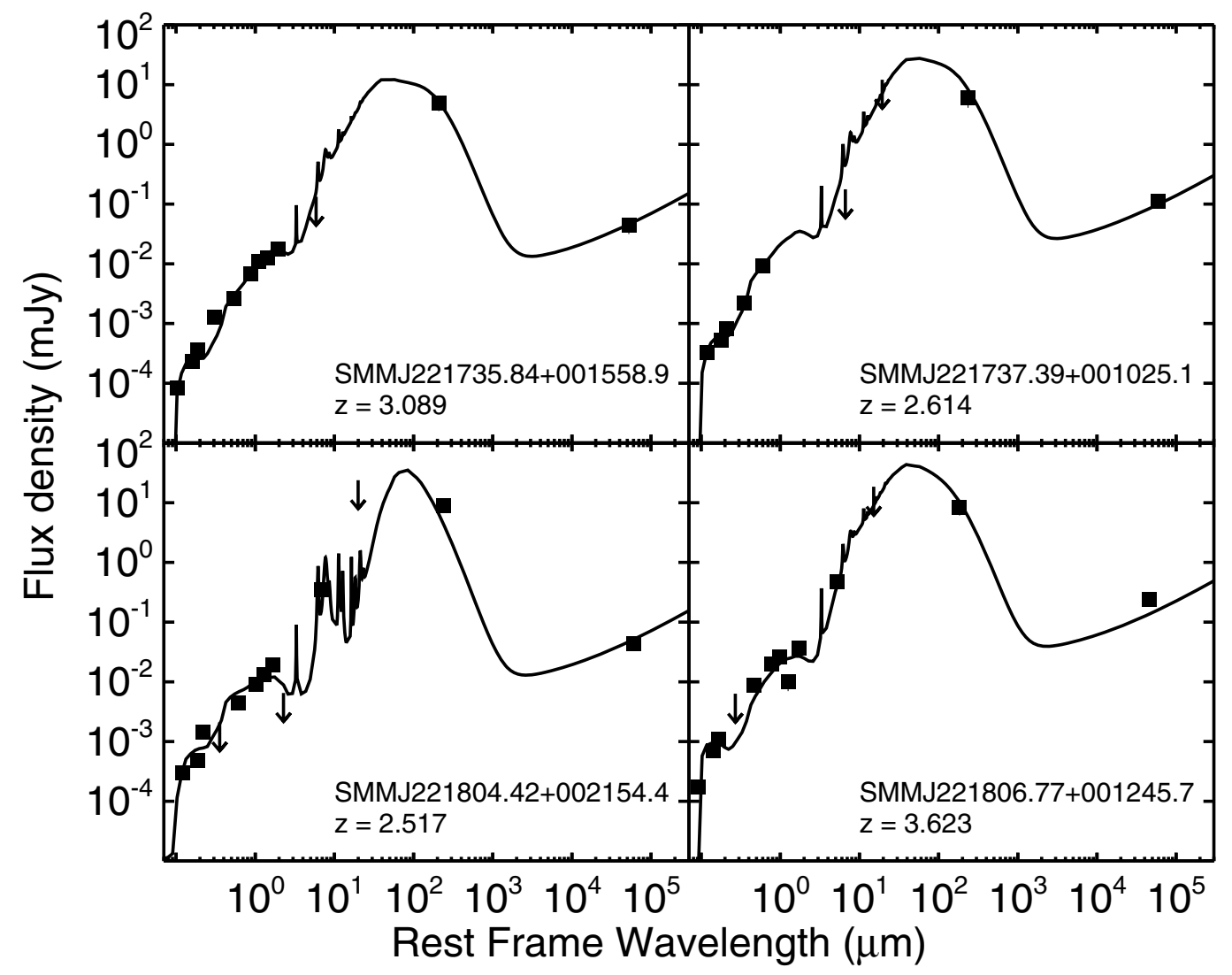

Fig. A.1. (continued). 
M. Michałowski et al.: Evolution of submillimeter galaxies

Table A.4. Compilation of star formation rate density determinations in $M_{\odot} \mathrm{yr}^{-1} \mathrm{Mpc}^{-3}$.

\begin{tabular}{|c|c|c|c|c|c|c|}
\hline$z$ & $\Delta z$ & $\rho_{\mathrm{SFR}}$ & $-\Delta \rho_{\mathrm{SFR}}$ & $+\Delta \rho_{\mathrm{SFR}}$ & Estimator & Reference \\
\hline 3.780 & 0.340 & 0.1690 & 0.0218 & 0.0250 & UV & Giavalisco et al. (2004); Hopkins (2004) \\
\hline 4.920 & 0.330 & 0.1089 & 0.0300 & 0.0414 & UV & Giavalisco et al. (2004); Hopkins (2004) \\
\hline 5.740 & 0.360 & 0.1194 & 0.0423 & 0.0655 & UV & Giavalisco et al. (2004); Hopkins (2004) \\
\hline 0.350 & 0.150 & 0.0356 & 0.0058 & 0.0070 & UV & Wilson et al. (2002); Hopkins (2004) \\
\hline 0.800 & 0.200 & 0.0656 & 0.0110 & 0.0133 & UV & Wilson et al. (2002); Hopkins (2004) \\
\hline 1.350 & 0.250 & 0.0925 & 0.0259 & 0.0361 & UV & Wilson et al. (2002); Hopkins (2004) \\
\hline 1.500 & 0.500 & 0.1954 & 0.0721 & 0.1143 & UV & Massarotti et al. (2001); Hopkins (2004) \\
\hline 2.750 & 0.750 & 0.3076 & 0.1135 & 0.1799 & UV & Massarotti et al. (2001); Hopkins (2004) \\
\hline 4.000 & 0.500 & 0.1300 & 0.0569 & 0.1012 & UV & Massarotti et al. (2001); Hopkins (2004) \\
\hline 0.150 & 0.150 & 0.0395 & 0.0043 & 0.0048 & UV & Sullivan et al. (2000); Hopkins (2004) \\
\hline 3.040 & 0.250 & 0.1603 & 0.0174 & 0.0196 & UV & Steidel et al. (1999); Hopkins (2004) \\
\hline 4.130 & 0.300 & 0.1245 & 0.0256 & 0.0322 & UV & Steidel et al. (1999); Hopkins (2004) \\
\hline 0.700 & 0.200 & 0.0481 & 0.0102 & 0.0130 & UV & Cowie et al. (1999); Hopkins (2004) \\
\hline 1.250 & 0.250 & 0.0652 & 0.0154 & 0.0201 & UV & Cowie et al. (1999); Hopkins (2004) \\
\hline 0.150 & 0.150 & 0.0428 & 0.0125 & 0.0176 & UV & Treyer et al. (1998); Hopkins (2004) \\
\hline 0.750 & 0.250 & 0.1019 & 0.0297 & 0.0420 & UV & Connolly et al. (1997); Hopkins (2004) \\
\hline 1.250 & 0.250 & 0.1368 & 0.0399 & 0.0564 & UV & Connolly et al. (1997); Hopkins (2004) \\
\hline 1.750 & 0.250 & 0.1062 & 0.0310 & 0.0438 & UV & Connolly et al. (1997); Hopkins (2004) \\
\hline 0.350 & 0.150 & 0.0289 & 0.0043 & 0.0051 & UV & Lilly et al. (1996); Hopkins (2004) \\
\hline 0.625 & 0.125 & 0.0542 & 0.0091 & 0.0110 & UV & Lilly et al. (1996); Hopkins (2004) \\
\hline 0.875 & 0.125 & 0.1050 & 0.0307 & 0.0433 & UV & Lilly et al. (1996); Hopkins (2004) \\
\hline 4.850 & 0.450 & 0.0350 & 0.0150 & 0.0150 & UV & Iwata et al. (2003); van Breukelen et al. (2005) \\
\hline 0.700 & 0.300 & 0.0462 & 0.0060 & 0.0068 & UV & Cowie et al. (1996); Somerville et al. (2001) \\
\hline 1.250 & 0.250 & 0.0668 & 0.0195 & 0.0276 & UV & Cowie et al. (1996); Somerville et al. (2001) \\
\hline 0.350 & 0.150 & 0.0495 & 0.0258 & 0.0272 & UV & Sawicki et al. (1997); Somerville et al. (2001) \\
\hline 0.750 & 0.250 & 0.0733 & 0.0109 & 0.0089 & UV & Sawicki et al. (1997); Somerville et al. (2001) \\
\hline 1.500 & 0.500 & 0.0988 & 0.0087 & 0.0095 & UV & Sawicki et al. (1997); Somerville et al. (2001) \\
\hline 2.500 & 0.500 & 0.2113 & 0.0273 & 0.0313 & UV & Sawicki et al. (1997); Somerville et al. (2001) \\
\hline 3.500 & 0.500 & 0.0922 & 0.0190 & 0.0187 & UV & Sawicki et al. (1997); Somerville et al. (2001) \\
\hline 0.250 & 0.250 & 0.0569 & 0.0226 & 0.0592 & UV & Pascarelle et al. (1998); Somerville et al. (2001) \\
\hline 0.750 & 0.250 & 0.0638 & 0.0176 & 0.0446 & UV & Pascarelle et al. (1998); Somerville et al. (2001) \\
\hline 1.250 & 0.250 & 0.0901 & 0.0218 & 0.0665 & UV & Pascarelle et al. (1998); Somerville et al. (2001) \\
\hline 1.750 & 0.250 & 0.0922 & 0.0223 & 0.0681 & UV & Pascarelle et al. (1998); Somerville et al. (2001) \\
\hline 2.500 & 0.500 & 0.0556 & 0.0213 & 0.0503 & UV & Pascarelle et al. (1998); Somerville et al. (2001) \\
\hline 3.500 & 0.500 & 0.0519 & 0.0240 & 0.0616 & UV & Pascarelle et al. (1998); Somerville et al. (2001) \\
\hline 4.500 & 0.500 & 0.0653 & 0.0374 & 0.1145 & UV & Pascarelle et al. (1998); Somerville et al. (2001) \\
\hline 5.500 & 0.500 & 0.0285 & 0.0166 & 0.0824 & UV & Pascarelle et al. (1998); Somerville et al. (2001) \\
\hline 0.315 & 0.115 & 0.0373 & 0.0005 & 0.0005 & UV & Mobasher et al. (2009) \\
\hline 0.540 & 0.110 & 0.0533 & 0.0005 & 0.0006 & UV & Mobasher et al. (2009) \\
\hline 0.765 & 0.115 & 0.0957 & 0.0006 & 0.0006 & UV & Mobasher et al. (2009) \\
\hline 0.990 & 0.110 & 0.1082 & 0.0006 & 0.0006 & UV & Mobasher et al. (2009) \\
\hline 3.800 & 0.350 & 0.0891 & 0.0097 & 0.0109 & UV & Bouwens et al. (2007) \\
\hline 5.000 & 0.350 & 0.0331 & 0.0043 & 0.0049 & UV & Bouwens et al. (2007) \\
\hline 5.900 & 0.300 & 0.0224 & 0.0038 & 0.0045 & UV & Bouwens et al. (2007) \\
\hline 4.000 & 0.500 & 0.0362 & 0.0050 & 0.0050 & UV & Ouchi et al. (2004) \\
\hline 4.700 & 0.500 & 0.0300 & 0.0175 & 0.0175 & UV & Ouchi et al. (2004) \\
\hline 4.900 & 0.300 & 0.0138 & 0.0069 & 0.0069 & UV & Ouchi et al. (2004) \\
\hline 4.000 & 0.500 & 0.0300 & 0.0025 & 0.0025 & UV & Ouchi et al. (2004) \\
\hline 4.700 & 0.500 & 0.0200 & 0.0088 & 0.0088 & UV & Ouchi et al. (2004) \\
\hline 4.900 & 0.300 & 0.0088 & 0.0044 & 0.0044 & UV & Ouchi et al. (2004) \\
\hline 5.850 & 0.250 & 0.0034 & 0.0014 & 0.0014 & UV & Stanway et al. (2003) \\
\hline 1.000 & 0.500 & 0.2080 & 0.0990 & 0.0990 & UV & Thompson et al. (2006) \\
\hline 2.000 & 0.500 & 0.3980 & 0.1800 & 0.1800 & UV & Thompson et al. (2006) \\
\hline 3.000 & 0.500 & 0.3220 & 0.1600 & 0.1600 & UV & Thompson et al. (2006) \\
\hline 4.000 & 0.500 & 0.0940 & 0.0390 & 0.0390 & UV & Thompson et al. (2006) \\
\hline 5.000 & 0.500 & 0.0410 & 0.0160 & 0.0160 & UV & Thompson et al. (2006) \\
\hline 6.000 & 0.500 & 0.1260 & 0.0740 & 0.0740 & UV & Thompson et al. (2006) \\
\hline 2.280 & 0.330 & 0.1778 & 0.1407 & 0.6733 & UV & Ly et al. (2009) \\
\hline 4.000 & 0.300 & 0.1301 & 0.0194 & 0.0227 & UV & Yoshida et al. (2006); Ly et al. (2009) \\
\hline 4.700 & 0.300 & 0.0745 & 0.0316 & 0.0550 & UV & Yoshida et al. (2006); Ly et al. (2009) \\
\hline 2.300 & 0.400 & 0.1500 & 0.0223 & 0.0262 & UV & Reddy et al. (2008); Ly et al. (2009) \\
\hline 0.050 & 0.050 & 0.0126 & 0.0026 & 0.0033 & UV & Wyder et al. (2005) \\
\hline 3.200 & 0.140 & 0.1600 & 0.0798 & 0.1592 & UV & Shim et al. (2007) \\
\hline 0.330 & 0.040 & 0.0353 & 0.0059 & 0.0071 & UV & Dahlen et al. (2007) \\
\hline 0.545 & 0.085 & 0.0996 & 0.0148 & 0.0174 & UV & Dahlen et al. (2007) \\
\hline
\end{tabular}


Table A.4. continued.

\begin{tabular}{|c|c|c|c|c|c|c|}
\hline$z$ & $\Delta z$ & $\rho_{\mathrm{SFR}}$ & $-\Delta \rho_{\mathrm{SFR}}$ & $+\Delta \rho_{\mathrm{SFR}}$ & Estimator & Reference \\
\hline 1.125 & 0.205 & 0.1283 & 0.0240 & 0.0295 & UV & Dahlen et al. (2007) \\
\hline 1.750 & 0.130 & 0.1898 & 0.0390 & 0.0491 & UV & Dahlen et al. (2007) \\
\hline 2.225 & 0.145 & 0.1407 & 0.0364 & 0.0491 & UV & Dahlen et al. (2007) \\
\hline 2.750 & 0.750 & 0.1800 & 0.0337 & 0.0414 & UV & Wadadekar et al. (2006) \\
\hline 0.900 & 0.500 & 0.0989 & 0.0221 & 0.0285 & [O 2] & Teplitz et al. (2003); Hopkins (2004) \\
\hline 0.025 & 0.025 & 0.0122 & 0.0036 & 0.0050 & [O 2] & Gallego et al. (2002); Hopkins (2004) \\
\hline 0.200 & 0.100 & 0.0136 & 0.0032 & 0.0062 & [O 2] & Hogg et al. (1998); Hopkins (2004) \\
\hline 0.300 & 0.100 & 0.0119 & 0.0023 & 0.0031 & [O 2] & Hogg et al. (1998); Hopkins (2004) \\
\hline 0.400 & 0.100 & 0.0536 & 0.0095 & 0.0147 & [O 2] & Hogg et al. (1998); Hopkins (2004) \\
\hline 0.500 & 0.100 & 0.0955 & 0.0137 & 0.0180 & [O 2] & Hogg et al. (1998); Hopkins (2004) \\
\hline 0.600 & 0.100 & 0.0649 & 0.0086 & 0.0117 & [O 2] & Hogg et al. (1998); Hopkins (2004) \\
\hline 0.700 & 0.100 & 0.0535 & 0.0083 & 0.0120 & [O 2] & Hogg et al. (1998); Hopkins (2004) \\
\hline 0.800 & 0.100 & 0.0566 & 0.0085 & 0.0116 & [O 2] & Hogg et al. (1998); Hopkins (2004) \\
\hline 0.900 & 0.100 & 0.0714 & 0.0112 & 0.0165 & [O 2] & Hogg et al. (1998); Hopkins (2004) \\
\hline 1.000 & 0.100 & 0.1146 & 0.0208 & 0.0320 & [O 2] & Hogg et al. (1998); Hopkins (2004) \\
\hline 1.100 & 0.100 & 0.0899 & 0.0242 & 0.0523 & [O 2] & Hogg et al. (1998); Hopkins (2004) \\
\hline 1.200 & 0.100 & 0.0859 & 0.0286 & 0.0859 & [O 2] & Hogg et al. (1998); Hopkins (2004) \\
\hline 0.375 & 0.125 & 0.0197 & 0.0033 & 0.0034 & [O 2] & Hammer et al. (1997); Hopkins (2004) \\
\hline 0.625 & 0.125 & 0.0594 & 0.0174 & 0.0171 & [O 2] & Hammer et al. (1997); Hopkins (2004) \\
\hline 0.875 & 0.125 & 0.1396 & 0.0814 & 0.0817 & [O 2] & Hammer et al. (1997); Hopkins (2004) \\
\hline 0.401 & 0.011 & 0.0240 & 0.0080 & 0.0080 & [O 3] & Hippelein et al. (2003) \\
\hline 0.636 & 0.010 & 0.0720 & 0.0160 & 0.0160 & [O 3] & Hippelein et al. (2003) \\
\hline 0.881 & 0.014 & 0.1070 & 0.0350 & 0.0350 & [O 2] & Hippelein et al. (2003) \\
\hline 1.193 & 0.018 & 0.2280 & 0.0550 & 0.0550 & [O 2] & Hippelein et al. (2003) \\
\hline 2.750 & 0.750 & 0.2773 & 0.0810 & 0.1144 & $\mathrm{H} \beta$ & Pettini et al. (1998); Hopkins (2004) \\
\hline 0.025 & 0.025 & 0.0249 & 0.0056 & 0.0072 & $\mathrm{H} \alpha$ & Pérez-González et al. (2003); Hopkins (2004) \\
\hline 0.800 & 0.300 & 0.1172 & 0.0262 & 0.0338 & $\mathrm{H} \alpha$ & Tresse et al. (2002); Hopkins (2004) \\
\hline 2.200 & 0.050 & 0.2655 & 0.0641 & 0.0845 & $\mathrm{H} \alpha$ & Moorwood et al. (2000); Hopkins (2004) \\
\hline 1.250 & 0.550 & 0.2350 & 0.0137 & 0.0145 & $\mathrm{H} \alpha$ & Hopkins et al. (2000); Hopkins (2004) \\
\hline 0.150 & 0.150 & 0.0151 & 0.0020 & 0.0022 & $\mathrm{H} \alpha$ & Sullivan et al. (2000); Hopkins (2004) \\
\hline 0.900 & 0.100 & 0.1067 & 0.0294 & 0.0440 & $\mathrm{H} \alpha$ & Glazebrook et al. (1999); Hopkins (2004) \\
\hline 1.300 & 0.600 & 0.2799 & 0.0676 & 0.0891 & $\mathrm{H} \alpha$ & Yan et al. (1999); Hopkins (2004) \\
\hline 0.200 & 0.100 & 0.0324 & 0.0042 & 0.0048 & $\mathrm{H} \alpha$ & Tresse \& Maddox (1998); Hopkins (2004) \\
\hline 0.022 & 0.022 & 0.0126 & 0.0046 & 0.0074 & $\mathrm{H} \alpha$ & Gallego et al. (1995); Hopkins (2004) \\
\hline 0.043 & 0.043 & 0.0240 & 0.0026 & 0.0029 & $\mathrm{H} \alpha$ & Gronwall (1999); Somerville et al. (2001) \\
\hline 0.243 & 0.009 & 0.0360 & 0.0120 & 0.0060 & $\mathrm{H} \alpha$ & Fujita et al. (2003b) \\
\hline 0.245 & 0.007 & 0.0240 & 0.0060 & 0.0060 & $\mathrm{H} \alpha$ & Hippelein et al. (2003) \\
\hline 0.100 & 0.100 & 0.0192 & 0.0042 & 0.0014 & $\mathrm{H} \alpha$ & Brinchmann et al. (2004) \\
\hline 0.010 & 0.010 & 0.0158 & 0.0033 & 0.0071 & $\mathrm{H} \alpha$ & Hanish et al. (2006) \\
\hline 0.840 & 0.030 & 0.1500 & 0.0200 & 0.0200 & $\mathrm{H} \alpha$ & Sobral et al. (2009) \\
\hline 2.230 & 0.150 & 0.1700 & 0.0900 & 0.1600 & $\mathrm{H} \alpha$ & Geach et al. (2008) \\
\hline 0.242 & 0.009 & 0.0180 & 0.0040 & 0.0070 & $\mathrm{H} \alpha$ & Shioya et al. (2008) \\
\hline 0.840 & 0.030 & 0.1700 & 0.0300 & 0.0300 & $\mathrm{H} \alpha$ & Villar et al. (2008) \\
\hline 2.300 & 0.400 & 0.3484 & 0.0869 & 0.0869 & $\mathrm{H} \alpha$ & Reddy et al. (2008) \\
\hline 3.050 & 0.350 & 0.2141 & 0.0450 & 0.0450 & $\mathrm{H} \alpha$ & Reddy et al. (2008) \\
\hline 0.350 & 0.150 & 0.0365 & 0.0169 & 0.0314 & mid-IR & Flores et al. (1999); Hopkins (2004) \\
\hline 0.625 & 0.125 & 0.0678 & 0.0297 & 0.0527 & mid-IR & Flores et al. (1999); Hopkins (2004) \\
\hline 0.875 & 0.125 & 0.1337 & 0.0602 & 0.1096 & mid-IR & Flores et al. (1999); Hopkins (2004) \\
\hline 0.215 & 0.215 & 0.0300 & 0.0100 & 0.0200 & mid-IR & Mann et al. (2002) \\
\hline 0.515 & 0.085 & 0.0700 & 0.0100 & 0.0200 & mid-IR & Mann et al. (2002) \\
\hline 0.100 & 0.100 & 0.0175 & 0.0049 & 0.0049 & mid-IR & Pozzi et al. (2004) \\
\hline 0.300 & 0.100 & 0.0301 & 0.0140 & 0.0140 & mid-IR & Pozzi et al. (2004) \\
\hline 0.300 & 0.100 & 0.0197 & 0.0067 & 0.0066 & mid-IR & Zheng et al. (2007) \\
\hline 0.500 & 0.100 & 0.0349 & 0.0088 & 0.0088 & mid-IR & Zheng et al. (2007) \\
\hline 0.700 & 0.100 & 0.0570 & 0.0096 & 0.0101 & mid-IR & Zheng et al. (2007) \\
\hline 0.900 & 0.100 & 0.0616 & 0.0120 & 0.0121 & mid-IR & Zheng et al. (2007) \\
\hline 2.300 & 0.400 & 0.2091 & 0.0357 & 0.0357 & mid-IR & Reddy et al. (2008) \\
\hline 3.050 & 0.350 & 0.1124 & 0.0211 & 0.0211 & mid-IR & Reddy et al. (2008) \\
\hline 1.000 & 0.100 & 0.2000 & 0.0300 & 0.0300 & mid-IR & Caputi et al. (2007) \\
\hline 2.000 & 0.300 & 0.1100 & 0.0200 & 0.0200 & mid-IR & Caputi et al. (2007) \\
\hline 0.450 & 0.150 & 0.2750 & 0.0220 & 0.0220 & mid-IR & Santini et al. (2009) \\
\hline 0.800 & 0.200 & 0.4870 & 0.0170 & 0.0170 & mid-IR & Santini et al. (2009) \\
\hline 1.250 & 0.250 & 0.7550 & 0.0290 & 0.0290 & mid-IR & Santini et al. (2009) \\
\hline 2.000 & 0.500 & 1.6590 & 0.0580 & 0.0580 & mid-IR & Santini et al. (2009) \\
\hline
\end{tabular}


M. Michałowski et al.: Evolution of submillimeter galaxies

Table A.4. continued.

\begin{tabular}{|c|c|c|c|c|c|c|}
\hline$z$ & $\Delta z$ & $\rho_{\mathrm{SFR}}$ & $-\Delta \rho_{\mathrm{SFR}}$ & $+\Delta \rho_{\mathrm{SFR}}$ & Estimator & Reference \\
\hline 0.100 & 0.100 & 0.0180 & 0.0025 & 0.0029 & mid-IR & Pérez-González et al. (2005) \\
\hline 0.100 & 0.100 & 0.0163 & 0.0023 & 0.0027 & mid-IR & Pérez-González et al. (2005) \\
\hline 0.100 & 0.100 & 0.0171 & 0.0024 & 0.0028 & mid-IR & Pérez-González et al. (2005) \\
\hline 0.300 & 0.100 & 0.0384 & 0.0019 & 0.0020 & mid-IR & Pérez-González et al. (2005) \\
\hline 0.300 & 0.100 & 0.0356 & 0.0017 & 0.0018 & mid-IR & Pérez-González et al. (2005) \\
\hline 0.300 & 0.100 & 0.0330 & 0.0016 & 0.0017 & mid-IR & Pérez-González et al. (2005) \\
\hline 0.500 & 0.100 & 0.0818 & 0.0060 & 0.0064 & mid-IR & Pérez-González et al. (2005) \\
\hline 0.500 & 0.100 & 0.0927 & 0.0089 & 0.0098 & mid-IR & Pérez-González et al. (2005) \\
\hline 0.500 & 0.100 & 0.0589 & 0.0043 & 0.0046 & mid-IR & Pérez-González et al. (2005) \\
\hline 0.700 & 0.100 & 0.1052 & 0.0052 & 0.0054 & mid-IR & Pérez-González et al. (2005) \\
\hline 0.700 & 0.100 & 0.1459 & 0.0140 & 0.0155 & mid-IR & Pérez-González et al. (2005) \\
\hline 0.700 & 0.100 & 0.0951 & 0.0047 & 0.0049 & mid-IR & Pérez-González et al. (2005) \\
\hline 0.900 & 0.100 & 0.1319 & 0.0580 & 0.1036 & mid-IR & Pérez-González et al. (2005) \\
\hline 0.900 & 0.100 & 0.1877 & 0.0852 & 0.1559 & mid-IR & Pérez-González et al. (2005) \\
\hline 0.900 & 0.100 & 0.1255 & 0.0552 & 0.0985 & mid-IR & Pérez-González et al. (2005) \\
\hline 1.200 & 0.200 & 0.1741 & 0.0127 & 0.0137 & mid-IR & Pérez-González et al. (2005) \\
\hline 1.200 & 0.200 & 0.2477 & 0.0400 & 0.0478 & mid-IR & Pérez-González et al. (2005) \\
\hline 1.200 & 0.200 & 0.1423 & 0.0136 & 0.0151 & mid-IR & Pérez-González et al. (2005) \\
\hline 1.600 & 0.200 & 0.1574 & 0.0151 & 0.0167 & mid-IR & Pérez-González et al. (2005) \\
\hline 1.600 & 0.200 & 0.2076 & 0.0379 & 0.0464 & mid-IR & Pérez-González et al. (2005) \\
\hline 1.600 & 0.200 & 0.1353 & 0.0130 & 0.0143 & mid-IR & Pérez-González et al. (2005) \\
\hline 2.000 & 0.200 & 0.1319 & 0.0344 & 0.0466 & mid-IR & Pérez-González et al. (2005) \\
\hline 2.000 & 0.200 & 0.2239 & 0.0705 & 0.1028 & mid-IR & Pérez-González et al. (2005) \\
\hline 2.000 & 0.200 & 0.1388 & 0.0362 & 0.0490 & mid-IR & Pérez-González et al. (2005) \\
\hline 2.400 & 0.200 & 0.1785 & 0.0432 & 0.0570 & mid-IR & Pérez-González et al. (2005) \\
\hline 2.400 & 0.200 & 0.3524 & 0.1109 & 0.1618 & mid-IR & Pérez-González et al. (2005) \\
\hline 2.400 & 0.200 & 0.2296 & 0.0556 & 0.0733 & mid-IR & Pérez-González et al. (2005) \\
\hline 0.698 & 0.618 & 0.0102 & 0.0014 & 0.0014 & submm & This work \\
\hline 1.775 & 0.367 & 0.0228 & 0.0027 & 0.0027 & submm & This work \\
\hline 2.357 & 0.209 & 0.0486 & 0.0054 & 0.0054 & submm & This work \\
\hline 3.101 & 0.522 & 0.0341 & 0.0040 & 0.0040 & submm & This work \\
\hline 2.000 & 1.000 & 0.1476 & 0.0607 & 0.0973 & submm & Barger et al. (2000); Hopkins (2004) \\
\hline 4.500 & 1.500 & 0.1901 & 0.1195 & 0.2454 & submm & Barger et al. (2000); Hopkins (2004) \\
\hline 0.057 & 0.041 & 0.0206 & 0.0016 & 0.0024 & submm & Pascale et al. (2009) \\
\hline 0.138 & 0.040 & 0.0292 & 0.0022 & 0.0040 & submm & Pascale et al. (2009) \\
\hline 0.250 & 0.073 & 0.0192 & 0.0026 & 0.0036 & submm & Pascale et al. (2009) \\
\hline 0.454 & 0.132 & 0.0511 & 0.0022 & 0.0067 & submm & Pascale et al. (2009) \\
\hline 0.824 & 0.239 & 0.0785 & 0.0073 & 0.0086 & submm & Pascale et al. (2009) \\
\hline 2.281 & 1.219 & 0.1104 & 0.0092 & 0.0140 & submm & Pascale et al. (2009) \\
\hline 0.005 & 0.005 & 0.0109 & 0.0007 & 0.0008 & radio & Condon et al. (2002); Hopkins (2004) \\
\hline 0.080 & 0.080 & 0.0187 & 0.0035 & 0.0038 & radio & Sadler et al. (2002); Hopkins (2004) \\
\hline 0.010 & 0.010 & 0.0177 & 0.0036 & 0.0036 & radio & Serjeant et al. (2002); Hopkins (2004) \\
\hline 0.070 & 0.070 & 0.0120 & 0.0025 & 0.0031 & radio & Machalski \& Godlowski (2000); Hopkins (2004) \\
\hline 0.206 & 0.196 & 0.0408 & 0.0157 & 0.0155 & radio & Haarsma et al. (2000); Hopkins (2004) \\
\hline 0.464 & 0.054 & 0.0667 & 0.0246 & 0.0254 & radio & Haarsma et al. (2000); Hopkins (2004) \\
\hline 0.623 & 0.075 & 0.0764 & 0.0344 & 0.0340 & radio & Haarsma et al. (2000); Hopkins (2004) \\
\hline 0.804 & 0.080 & 0.1315 & 0.0446 & 0.0459 & radio & Haarsma et al. (2000); Hopkins (2004) \\
\hline 1.600 & 0.640 & 0.1641 & 0.0557 & 0.0522 & radio & Haarsma et al. (2000); Hopkins (2004) \\
\hline 0.005 & 0.005 & 0.0209 & 0.0000 & 0.0000 & radio & Condon (1989); Hopkins (2004) \\
\hline 0.152 & 0.149 & 0.0220 & 0.0010 & 0.0010 & radio & Mauch \& Sadler (2007) \\
\hline 0.310 & 0.210 & 0.0331 & 0.0074 & 0.0076 & radio & Seymour et al. (2008) \\
\hline 0.810 & 0.290 & 0.0851 & 0.0262 & 0.0271 & radio & Seymour et al. (2008) \\
\hline 1.500 & 0.400 & 0.1479 & 0.0666 & 0.0812 & radio & Seymour et al. (2008) \\
\hline 2.450 & 0.550 & 0.1202 & 0.0756 & 0.1036 & radio & Seymour et al. (2008) \\
\hline 0.100 & 0.100 & 0.0087 & 0.0063 & 0.0062 & radio & Dunne et al. (2009) \\
\hline 0.300 & 0.100 & 0.0292 & 0.0107 & 0.0106 & radio & Dunne et al. (2009) \\
\hline 0.500 & 0.100 & 0.0385 & 0.0118 & 0.0106 & radio & Dunne et al. (2009) \\
\hline 0.700 & 0.100 & 0.0700 & 0.0175 & 0.0164 & radio & Dunne et al. (2009) \\
\hline 0.900 & 0.100 & 0.0781 & 0.0202 & 0.0172 & radio & Dunne et al. (2009) \\
\hline 1.100 & 0.100 & 0.1124 & 0.0262 & 0.0249 & radio & Dunne et al. (2009) \\
\hline 1.300 & 0.100 & 0.1327 & 0.0287 & 0.0257 & radio & Dunne et al. (2009) \\
\hline 1.500 & 0.100 & 0.2043 & 0.0369 & 0.0343 & radio & Dunne et al. (2009) \\
\hline 1.700 & 0.100 & 0.1788 & 0.0339 & 0.0277 & radio & Dunne et al. (2009) \\
\hline 1.900 & 0.100 & 0.1582 & 0.0256 & 0.0245 & radio & Dunne et al. (2009) \\
\hline 2.250 & 0.250 & 0.1061 & 0.0141 & 0.0125 & radio & Dunne et al. (2009) \\
\hline 2.750 & 0.250 & 0.1049 & 0.0150 & 0.0123 & radio & Dunne et al. (2009) \\
\hline
\end{tabular}


Table A.4. continued.

\begin{tabular}{|c|c|c|c|c|c|c|}
\hline$z$ & $\Delta z$ & $\rho_{\mathrm{SFR}}$ & $-\Delta \rho_{\mathrm{SFR}}$ & $+\Delta \rho_{\mathrm{SFR}}$ & Estimator & Reference \\
\hline 3.250 & 0.250 & 0.0583 & 0.0078 & 0.0069 & radio & Dunne et al. (2009) \\
\hline 4.250 & 0.750 & 0.0184 & 0.0032 & 0.0026 & radio & Dunne et al. (2009) \\
\hline 0.150 & 0.150 & 0.0383 & 0.0131 & 0.0251 & X-ray & Georgakakis et al. (2003); Hopkins (2004) \\
\hline 2.750 & 0.750 & $>0.0607$ & & & UV & Madau et al. (1996); Hopkins (2004) \\
\hline 4.000 & 0.500 & $>0.0189$ & & & UV & Madau et al. (1996); Hopkins (2004) \\
\hline 2.750 & 0.750 & $>0.0290$ & & & UV & Madau et al. (1998); van Breukelen et al. (2005) \\
\hline 4.000 & 0.500 & $>0.0110$ & & & UV & Madau et al. (1998); van Breukelen et al. (2005) \\
\hline 3.200 & 0.140 & $>0.0033$ & & & UV & Shim et al. (2007) \\
\hline 2.200 & 0.350 & $>0.0372$ & & & UV & Sawicki \& Thompson (2006a,b); Ly et al. (2009) \\
\hline 2.960 & 0.260 & $>0.0370$ & & & UV & Sawicki \& Thompson (2006a,b); Ly et al. (2009) \\
\hline 4.130 & 0.260 & $>0.0161$ & & & UV & Sawicki \& Thompson (2006a,b); Ly et al. (2009) \\
\hline 3.500 & 0.500 & $>0.0442$ & & & UV & Paltani et al. (2007); Ly et al. (2009) \\
\hline 2.700 & 0.700 & $>0.0282$ & & & UV & Bouwens et al. (2003a) \\
\hline 3.850 & 0.450 & $>0.0166$ & & & UV & Bouwens et al. (2003a) \\
\hline 4.700 & 0.200 & $>0.0147$ & & & UV & Bouwens et al. (2003a) \\
\hline 6.000 & 0.200 & $>0.0360$ & & & UV & Bouwens et al. (2003b) \\
\hline 5.900 & 0.200 & $>0.0070$ & & & UV & Bouwens et al. (2004) \\
\hline 5.900 & 0.200 & $>0.0073$ & & & UV & Bouwens et al. (2006) \\
\hline 5.900 & 0.200 & $>0.0221$ & & & UV & Bouwens et al. (2006) \\
\hline 6.000 & 0.400 & $>0.0050$ & & & UV & Bunker et al. (2004) \\
\hline 3.050 & 0.350 & $>0.0321$ & & & UV & Reddy et al. (2008) \\
\hline 5.000 & 0.500 & $>0.0137$ & & & UV & Iwata et al. (2007) \\
\hline 5.900 & 0.300 & $>0.0003$ & & & UV & Shimasaku et al. (2005) \\
\hline 5.850 & 0.250 & $>0.0034$ & & & UV & Stanway et al. (2003) \\
\hline 2.259 & 0.053 & $>0.0054$ & & & $\operatorname{Ly} \alpha$ & Nilsson et al. (2009) \\
\hline 2.379 & 0.023 & $>0.0024$ & & & $\operatorname{Ly} \alpha$ & Palunas et al. (2004) \\
\hline 3.110 & 0.020 & $>0.0120$ & & & Ly $\alpha$ & Gronwall et al. (2007) \\
\hline 3.156 & 0.025 & $>0.0130$ & & & Ly $\alpha$ & Nilsson et al. (2007) \\
\hline 3.135 & 0.045 & $>0.0043$ & & & $\operatorname{Ly} \alpha$ & Ouchi et al. (2008) \\
\hline 3.140 & 0.040 & $>0.0300$ & & & $\operatorname{Ly} \alpha$ & Kudritzki et al. (2000) \\
\hline 3.400 & 0.030 & $>0.0060$ & & & $\operatorname{Ly} \alpha$ & Hu et al. (1998) \\
\hline 3.438 & 0.033 & $>0.0100$ & & & $\operatorname{Ly} \alpha$ & Cowie \& Hu (1998) \\
\hline 3.463 & 0.982 & $>0.0220$ & & & $\operatorname{Ly} \alpha$ & van Breukelen et al. (2005) \\
\hline 3.690 & 0.060 & $>0.0021$ & & & $\operatorname{Ly} \alpha$ & Ouchi et al. (2008) \\
\hline 3.700 & 0.220 & $>0.0004$ & & & $\operatorname{Ly} \alpha$ & Fujita et al. (2003a); van Breukelen et al. (2005) \\
\hline 4.500 & 0.064 & $>0.0100$ & & & $\operatorname{Ly} \alpha$ & Hu et al. (1998) \\
\hline 4.860 & 0.030 & $>0.0063$ & & & $\operatorname{Ly} \alpha$ & Ouchi et al. (2003); van Breukelen et al. (2005) \\
\hline 5.690 & 0.090 & $>0.0032$ & & & $\operatorname{Ly} \alpha$ & Ouchi et al. (2008) \\
\hline 5.700 & 0.100 & $>0.0012$ & & & $\operatorname{Ly} \alpha$ & Ajiki et al. (2003) \\
\hline 5.700 & 0.050 & $>0.0018$ & & & $\operatorname{Ly} \alpha$ & Malhotra \& Rhoads (2004) \\
\hline 5.700 & 0.050 & $>0.0023$ & & & $\operatorname{Ly} \alpha$ & Shimasaku et al. (2006) \\
\hline 5.700 & 0.050 & $>0.0007$ & & & $\operatorname{Ly} \alpha$ & Murayama et al. (2007) \\
\hline 5.735 & 0.062 & $>0.0005$ & & & $\operatorname{Ly} \alpha$ & Rhoads et al. (2003) \\
\hline 6.500 & 0.050 & $>0.0036$ & & & $\operatorname{Ly} \alpha$ & Malhotra \& Rhoads (2004) \\
\hline 6.550 & 0.050 & $>0.0006$ & & & $\operatorname{Ly} \alpha$ & Taniguchi et al. (2005) \\
\hline 6.578 & 0.002 & $>0.0005$ & & & $\operatorname{Ly} \alpha$ & Kodaira et al. (2003) \\
\hline 3.000 & 1.000 & $>0.0818$ & & & submm & Hughes et al. (1998); Hopkins (2004) \\
\hline
\end{tabular}

Notes. Lower limits indicate value not corrected for extinction. The data with double reference were taken directly form the compilation given in the second reference. . 
M. Michałowski et al.: Evolution of submillimeter galaxies

Table A.5. Compilation of stellar mass density determinations in $\log M_{\odot} \mathrm{Mpc}^{-3}$.

\begin{tabular}{|c|c|c|c|c|c|}
\hline$z$ & $\Delta z$ & $\rho_{*}$ & $-\Delta \rho_{*}$ & $+\Delta \rho_{*}$ & Reference \\
\hline 0.698 & 0.618 & 7.12 & 0.08 & 0.13 & This work \\
\hline 1.775 & 0.367 & 7.18 & 0.11 & 0.16 & This work \\
\hline 2.357 & 0.209 & 7.61 & 0.08 & 0.12 & This work \\
\hline 3.101 & 0.522 & 7.28 & 0.07 & 0.12 & This work \\
\hline 0.100 & 0.100 & 8.48 & 0.10 & 0.10 & Borch et al. (2006) \\
\hline 0.300 & 0.100 & 8.34 & 0.15 & 0.15 & Borch et al. (2006) \\
\hline 0.500 & 0.100 & 8.32 & 0.11 & 0.11 & Borch et al. (2006) \\
\hline 0.700 & 0.100 & 8.33 & 0.10 & 0.10 & Borch et al. (2006) \\
\hline 0.900 & 0.100 & 8.17 & 0.18 & 0.18 & Borch et al. (2006) \\
\hline 0.100 & 0.100 & 8.49 & 0.05 & 0.04 & Rudnick et al. (2003) \\
\hline 1.120 & 0.480 & 8.14 & 0.10 & 0.11 & Rudnick et al. (2003) \\
\hline 2.010 & 0.400 & 7.48 & 0.16 & 0.12 & Rudnick et al. (2003) \\
\hline 2.800 & 0.400 & 7.49 & 0.14 & 0.12 & Rudnick et al. (2003) \\
\hline 0.950 & 0.450 & 8.46 & 0.07 & 0.07 & Dickinson et al. (2003) \\
\hline 1.700 & 0.300 & 8.06 & 0.13 & 0.17 & Dickinson et al. (2003) \\
\hline 2.250 & 0.250 & 7.58 & 0.07 & 0.11 & Dickinson et al. (2003) \\
\hline 2.750 & 0.250 & 7.52 & 0.14 & 0.23 & Dickinson et al. (2003) \\
\hline 0.950 & 0.450 & 8.61 & 0.07 & 0.07 & Dickinson et al. (2003) \\
\hline 1.700 & 0.300 & 8.22 & 0.12 & 0.16 & Dickinson et al. (2003) \\
\hline 2.250 & 0.250 & 8.01 & 0.08 & 0.09 & Dickinson et al. (2003) \\
\hline 2.750 & 0.250 & 7.89 & 0.15 & 0.20 & Dickinson et al. (2003) \\
\hline 0.950 & 0.450 & 8.52 & 0.08 & 0.07 & Dickinson et al. (2003) \\
\hline 1.700 & 0.300 & 7.97 & 0.17 & 0.17 & Dickinson et al. (2003) \\
\hline 2.250 & 0.250 & 7.36 & 0.08 & 0.11 & Dickinson et al. (2003) \\
\hline 2.750 & 0.250 & 7.27 & 0.18 & 0.27 & Dickinson et al. (2003) \\
\hline 0.375 & 0.125 & 8.65 & 0.17 & 0.12 & Cohen (2002) \\
\hline 0.650 & 0.150 & 8.65 & 0.01 & 0.08 & Cohen (2002) \\
\hline 0.925 & 0.125 & 8.62 & 0.09 & 0.08 & Cohen (2002) \\
\hline 0.500 & 0.100 & 8.83 & 0.04 & 0.04 & Drory et al. $(2004,2005)$ \\
\hline 0.700 & 0.100 & 8.76 & 0.04 & 0.04 & Drory et al. $(2004,2005)$ \\
\hline 0.900 & 0.100 & 8.60 & 0.04 & 0.04 & Drory et al. $(2004,2005)$ \\
\hline 1.100 & 0.100 & 8.55 & 0.04 & 0.04 & Drory et al. $(2004,2005)$ \\
\hline 0.500 & 0.250 & 8.50 & 0.27 & 0.27 & Drory et al. $(2004,2005)$ \\
\hline 0.500 & 0.250 & 8.51 & 0.17 & 0.17 & Drory et al. $(2004,2005)$ \\
\hline 1.000 & 0.250 & 8.42 & 0.12 & 0.12 & Drory et al. $(2004,2005)$ \\
\hline 1.000 & 0.250 & 8.29 & 0.19 & 0.19 & Drory et al. $(2004,2005)$ \\
\hline 1.500 & 0.250 & 8.38 & 0.16 & 0.16 & Drory et al. $(2004,2005)$ \\
\hline 1.500 & 0.250 & 8.03 & 0.16 & 0.16 & Drory et al. $(2004,2005)$ \\
\hline 2.000 & 0.250 & 8.09 & 0.19 & 0.19 & Drory et al. $(2004,2005)$ \\
\hline 2.000 & 0.250 & 8.04 & 0.20 & 0.20 & Drory et al. $(2004,2005)$ \\
\hline 2.625 & 0.375 & 8.15 & 0.19 & 0.19 & Drory et al. $(2004,2005)$ \\
\hline 2.625 & 0.375 & 7.78 & 0.20 & 0.20 & Drory et al. $(2004,2005)$ \\
\hline 3.500 & 0.500 & 7.92 & 0.17 & 0.17 & Drory et al. $(2004,2005)$ \\
\hline 3.500 & 0.500 & 7.68 & 0.20 & 0.20 & Drory et al. $(2004,2005)$ \\
\hline 4.500 & 0.500 & 7.37 & 0.26 & 0.26 & Drory et al. $(2004,2005)$ \\
\hline 4.500 & 0.500 & 7.43 & 0.20 & 0.20 & Drory et al. $(2004,2005)$ \\
\hline 0.900 & 0.100 & 8.18 & 0.13 & 0.10 & Glazebrook et al. (2004) \\
\hline 1.200 & 0.100 & 7.82 & 0.13 & 0.01 & Glazebrook et al. (2004) \\
\hline 1.450 & 0.150 & 8.08 & 0.09 & 0.08 & Glazebrook et al. (2004) \\
\hline 1.800 & 0.200 & 7.69 & 0.13 & 0.11 & Glazebrook et al. (2004) \\
\hline 0.500 & 0.100 & 8.32 & 0.03 & 0.03 & Fontana et al. $(2003,2004,2006)$ \\
\hline 0.700 & 0.100 & 8.53 & 0.02 & 0.02 & Fontana et al. (2003, 2004, 2006) \\
\hline 0.900 & 0.100 & 8.16 & 0.03 & 0.03 & Fontana et al. $(2003,2004,2006)$ \\
\hline 1.150 & 0.150 & 8.26 & 0.02 & 0.02 & Fontana et al. $(2003,2004,2006)$ \\
\hline 1.450 & 0.150 & 7.96 & 0.03 & 0.03 & Fontana et al. $(2003,2004,2006)$ \\
\hline 1.800 & 0.200 & 7.90 & 0.04 & 0.04 & Fontana et al. $(2003,2004,2006)$ \\
\hline 2.500 & 0.500 & 7.60 & 0.04 & 0.04 & Fontana et al. $(2003,2004,2006)$ \\
\hline 3.500 & 0.500 & 7.23 & 0.12 & 0.12 & Fontana et al. $(2003,2004,2006)$ \\
\hline 4.500 & 0.500 & 7.73 & 0.12 & 0.12 & Fontana et al. $(2003,2004,2006)$ \\
\hline 5.500 & 0.500 & 7.84 & 0.12 & 0.12 & Fontana et al. $(2003,2004,2006)$ \\
\hline 0.450 & 0.250 & 8.51 & 0.04 & 0.24 & Fontana et al. $(2003,2004,2006)$ \\
\hline 0.850 & 0.150 & 8.44 & 0.05 & 0.20 & Fontana et al. $(2003,2004,2006)$ \\
\hline 1.250 & 0.250 & 8.19 & 0.11 & 0.13 & Fontana et al. $(2003,2004,2006)$ \\
\hline 1.750 & 0.250 & 7.86 & 0.24 & 0.24 & Fontana et al. $(2003,2004,2006)$ \\
\hline 2.250 & 0.250 & 7.65 & 0.24 & 0.24 & Fontana et al. $(2003,2004,2006)$ \\
\hline 0.500 & 0.250 & 8.64 & 0.17 & 0.24 & Fontana et al. $(2003,2004,2006)$ \\
\hline
\end{tabular}


A\&A 514, A67 (2010)

Table A.5. Compilation of stellar mass density determinations in $\log M_{\odot} \mathrm{Mpc}^{-3}$.

\begin{tabular}{|c|c|c|c|c|c|}
\hline$z$ & $\Delta z$ & $\rho_{*}$ & $-\Delta \rho_{*}$ & $+\Delta \rho_{*}$ & Reference \\
\hline 1.000 & 0.250 & 8.29 & 0.31 & 0.35 & Fontana et al. (2003, 2004, 2006) \\
\hline 1.625 & 0.375 & 7.87 & 0.28 & 0.35 & Fontana et al. $(2003,2004,2006)$ \\
\hline 2.250 & 0.250 & 7.92 & 0.42 & 0.26 & Fontana et al. $(2003,2004,2006)$ \\
\hline 2.850 & 0.350 & 7.90 & 0.20 & 0.38 & Fontana et al. (2003, 2004, 2006) \\
\hline 0.100 & 0.100 & 8.75 & 0.07 & 0.06 & Cole et al. (2001) \\
\hline 0.350 & 0.150 & 8.53 & 0.13 & 0.09 & Brinchmann \& Ellis (2000) \\
\hline 0.625 & 0.125 & 8.56 & 0.05 & 0.06 & Brinchmann \& Ellis (2000) \\
\hline 0.875 & 0.125 & 8.48 & 0.04 & 0.06 & Brinchmann \& Ellis (2000) \\
\hline 0.100 & 0.100 & 8.75 & 0.12 & 0.12 & Pérez-González et al. (2008) \\
\hline 0.300 & 0.100 & 8.61 & 0.06 & 0.06 & Pérez-González et al. (2008) \\
\hline 0.500 & 0.100 & 8.57 & 0.04 & 0.04 & Pérez-González et al. (2008) \\
\hline 0.700 & 0.100 & 8.52 & 0.05 & 0.05 & Pérez-González et al. (2008) \\
\hline 0.900 & 0.100 & 8.44 & 0.05 & 0.05 & Pérez-González et al. (2008) \\
\hline 1.150 & 0.150 & 8.35 & 0.05 & 0.05 & Pérez-González et al. (2008) \\
\hline 1.450 & 0.150 & 8.18 & 0.07 & 0.07 & Pérez-González et al. (2008) \\
\hline 1.800 & 0.200 & 8.02 & 0.07 & 0.07 & Pérez-González et al. (2008) \\
\hline 2.250 & 0.250 & 7.87 & 0.09 & 0.09 & Pérez-González et al. (2008) \\
\hline 2.750 & 0.250 & 7.76 & 0.18 & 0.18 & Pérez-González et al. (2008) \\
\hline 3.250 & 0.250 & 7.63 & 0.14 & 0.14 & Pérez-González et al. (2008) \\
\hline 3.750 & 0.250 & 7.49 & 0.13 & 0.13 & Pérez-González et al. (2008) \\
\hline 0.150 & 0.150 & 8.76 & 0.13 & 0.11 & Salucci \& Persic (1999) \\
\hline 0.097 & 0.084 & 8.73 & 0.07 & 0.06 & Driver et al. (2007) \\
\hline 0.150 & 0.150 & 8.72 & 0.01 & 0.01 & Bell et al. (2003) \\
\hline 0.950 & 0.450 & 8.45 & 0.07 & 0.06 & Conselice et al. (2005) \\
\hline 1.700 & 0.300 & 7.74 & 0.22 & 0.15 & Conselice et al. (2005) \\
\hline 2.250 & 0.250 & 7.36 & 0.40 & 0.21 & Conselice et al. (2005) \\
\hline 2.750 & 0.250 & 7.30 & 0.92 & 0.27 & Conselice et al. (2005) \\
\hline 0.500 & 0.250 & 8.57 & 0.03 & 0.03 & Elsner et al. (2008) \\
\hline 1.000 & 0.250 & 8.37 & 0.02 & 0.02 & Elsner et al. (2008) \\
\hline 1.500 & 0.250 & 8.22 & 0.03 & 0.03 & Elsner et al. (2008) \\
\hline 2.000 & 0.250 & 8.10 & 0.04 & 0.04 & Elsner et al. (2008) \\
\hline 2.500 & 0.250 & 7.93 & 0.04 & 0.04 & Elsner et al. (2008) \\
\hline 3.500 & 0.500 & 7.59 & 0.05 & 0.05 & Elsner et al. (2008) \\
\hline 4.500 & 0.500 & 6.90 & 0.08 & 0.08 & Elsner et al. (2008) \\
\hline 3.960 & 0.290 & 7.01 & 0.06 & 0.05 & Stark et al. (2009) \\
\hline 4.790 & 0.250 & 6.63 & 0.07 & 0.06 & Stark et al. (2009) \\
\hline 6.010 & 0.250 & 6.29 & 0.09 & 0.07 & Stark et al. (2009) \\
\hline 5.000 & 0.600 & 6.78 & 0.08 & 0.22 & Stark et al. (2007) \\
\hline 6.000 & 0.300 & 6.40 & 0.00 & 0.51 & Eyles et al. (2007) \\
\hline 6.000 & 0.500 & 6.59 & 0.55 & 0.24 & Yan et al. (2006) \\
\hline 0.100 & 0.100 & 8.59 & 0.04 & 0.04 & Rudnick et al. (2006) \\
\hline 0.500 & 0.500 & 8.05 & 0.03 & 0.07 & Rudnick et al. (2006) \\
\hline 1.300 & 0.300 & 7.87 & 0.04 & 0.07 & Rudnick et al. (2006) \\
\hline 2.000 & 0.400 & 7.76 & 0.06 & 0.06 & Rudnick et al. (2006) \\
\hline 2.800 & 0.400 & 7.59 & 0.11 & 0.06 & Rudnick et al. (2006) \\
\hline 0.100 & 0.100 & 8.51 & 0.07 & 0.07 & Marchesini et al. (2009) \\
\hline 1.650 & 0.350 & 7.91 & 0.15 & 0.02 & Marchesini et al. (2009) \\
\hline 2.500 & 0.500 & 7.55 & 0.18 & 0.12 & Marchesini et al. (2009) \\
\hline 3.500 & 0.500 & 7.27 & 0.39 & 0.93 & Marchesini et al. (2009) \\
\hline 0.550 & 0.150 & 8.31 & 0.07 & 0.07 & Bundy et al. (2006) \\
\hline 0.875 & 0.125 & 8.30 & 0.10 & 0.10 & Bundy et al. (2006) \\
\hline 1.200 & 0.200 & 8.15 & 0.10 & 0.10 & Bundy et al. (2006) \\
\hline 0.300 & 0.100 & 8.46 & 0.03 & 0.03 & Ilbert et al. (2010) \\
\hline 0.500 & 0.100 & 8.22 & 0.02 & 0.02 & Ilbert et al. (2010) \\
\hline 0.700 & 0.100 & 8.25 & 0.02 & 0.02 & Ilbert et al. (2010) \\
\hline 0.900 & 0.100 & 8.32 & 0.01 & 0.01 & Ilbert et al. (2010) \\
\hline 1.100 & 0.100 & 8.09 & 0.02 & 0.02 & Ilbert et al. (2010) \\
\hline 1.350 & 0.150 & 7.93 & 0.01 & 0.01 & Ilbert et al. (2010) \\
\hline 1.750 & 0.250 & 7.72 & 0.09 & 0.14 & Ilbert et al. (2010) \\
\hline 0.300 & 0.100 & 8.79 & 0.17 & 0.15 & Arnouts et al. (2007) \\
\hline 0.500 & 0.100 & 8.63 & 0.11 & 0.12 & Arnouts et al. (2007) \\
\hline
\end{tabular}


M. Michałowski et al.: Evolution of submillimeter galaxies

Table A.5. continued.

\begin{tabular}{cccccl}
\hline \hline$z$ & $\Delta z$ & $\rho_{*}$ & $-\Delta \rho_{*}$ & $+\Delta \rho_{*}$ & Reference \\
\hline 0.700 & 0.100 & 8.63 & 0.10 & 0.10 & Arnouts et al. (2007) \\
0.900 & 0.100 & 8.73 & 0.13 & 0.13 & Arnouts et al. (2007) \\
1.100 & 0.100 & 8.54 & 0.11 & 0.11 & Arnouts et al. (2007) \\
1.350 & 0.150 & 8.43 & 0.12 & 0.12 & Arnouts et al. (2007) \\
1.750 & 0.250 & 8.17 & 0.12 & 0.12 & Arnouts et al. (2007) \\
0.325 & 0.225 & 8.66 & 0.10 & 0.10 & Franceschini et al. (2006) \\
0.725 & 0.175 & 8.61 & 0.10 & 0.10 & Franceschini et al. (2006) \\
1.150 & 0.250 & 8.45 & 0.10 & 0.10 & Franceschini et al. (2006) \\
0.225 & 0.175 & 8.45 & 0.01 & 0.01 & Pozzetti et al. (2007) \\
0.550 & 0.150 & 8.34 & 0.02 & 0.02 & Pozzetti et al. (2007) \\
0.800 & 0.100 & 8.22 & 0.01 & 0.01 & Pozzetti et al. (2007) \\
1.050 & 0.150 & 8.14 & 0.01 & 0.01 & Pozzetti et al. (2007) \\
1.400 & 0.200 & 8.04 & 0.02 & 0.02 & Pozzetti et al. (2007) \\
2.050 & 0.450 & 8.05 & 0.01 & 0.01 & Pozzetti et al. (2007) \\
0.025 & 0.025 & 8.81 & 0.05 & 0.05 & Kochanek et al. (2001) \\
0.100 & 0.100 & 8.72 & 0.03 & 0.03 & Driver et al. (2006) \\
1.100 & 0.400 & 8.47 & 0.11 & 0.11 & Gwyn \& Hartwick (2005) \\
1.750 & 0.250 & 8.38 & 0.21 & 0.21 & Gwyn \& Hartwick (2005) \\
2.500 & 0.500 & 8.21 & 0.14 & 0.14 & Gwyn \& Hartwick (2005) \\
4.500 & 1.500 & 7.93 & 0.11 & 0.11 & Gwyn \& Hartwick (2005) \\
1.250 & 0.250 & 8.37 & 0.07 & 0.06 & Caputi et al. (2006) \\
1.750 & 0.250 & 8.12 & 0.07 & 0.06 & Caputi et al. (2006) \\
\hline
\end{tabular}

